九州大学学術情報リポジトリ

Kyushu University Institutional Repository

\title{
Decay properties of solutions to the linearized compressible Navier-Stokes equation around time-periodic parallel flow
}

Brezina, Jan

Graduate School of Mathematics, Kyushu University

Kage i, Yoshiyuki

Faculty of Mathematics, Kyushu University

http://hdl. handle. net/2324/19412

出版情報 : MI Preprint Series. 2011-8，2012-07. World Scientific バージョン:

権利関係: Preprint of an article submitted for consideration in Mathematical Models and Methods in Applied Sciences o 2012 World Scientific Publishing Company

[ht tp://www. worldscient ific. com/worldscinet/m3as] 


\section{Preprint Series}

Kyushu University

The Global COE Program

Math-for-Industry Education \& Research Hub

\section{Decay properties of solutions to the linearized compressible Navier-Stokes equation around time-periodic parallel flow}

\section{Jan Brezina \& Yoshiyuki Kagei}

MI 2011-8

( Received March 30, 2011 )

Faculty of Mathematics

Kyushu University

Fukuoka, JAPAN 


\title{
Decay properties of solutions to the linearized compressible Navier-Stokes equation around time-periodic parallel flow
}

\author{
Jan Březina * $\quad$ Yoshiyuki Kagei ${ }^{\dagger}$
}

\begin{abstract}
Decay estimates on solutions to the linearized compressible Navier-Stokes equation around time-periodic parallel flow are established. It is shown that if the Reynolds and Mach numbers are sufficiently small, solutions of the linearized problem decay in $L^{2}$ norm as an $n-1$ dimensional heat kernel. Furthermore, it is proved that the asymptotic leading part of solutions is given by solutions of an $n-1$ dimensional linear heat equation with a convective term multiplied by time-periodic function.
\end{abstract}

\section{Mathematics Subject Classification}

Keywords. Compressible Navier-Stokes equation, decay estimates, asymptotic behavior, time-periodic.

\section{Introduction}

This paper is concerned with the asymptotic behavior of solutions to the compressible NavierStokes equation with time-periodic external force and (or) time-periodic boundary conditions.

We consider the system of equations

$$
\begin{gathered}
\partial_{t} \rho+\operatorname{div}(\rho v)=0, \\
\rho\left(\partial_{t} v+v \cdot \nabla v\right)-\mu \Delta v-\left(\mu+\mu^{\prime}\right) \nabla \operatorname{div} v+\nabla P(\rho)=\rho \boldsymbol{g},
\end{gathered}
$$

in an $n$ dimensional infinite layer $\Omega_{\ell}=\mathbb{R}^{n-1} \times(0, \ell)$ :

$$
\begin{aligned}
\Omega_{\ell}=\{x & =\left(x^{\prime}, x_{n}\right) ; \\
& \left.x^{\prime}=\left(x_{1}, \ldots, x_{n-1}\right) \in \mathbb{R}^{n-1}, 0<x_{n}<\ell\right\} .
\end{aligned}
$$

Here $n \geq 2 ; \rho=\rho(x, t)$ and $v=\left(v^{1}(x, t), \ldots, v^{n}(x, t)\right)$ denote the unknown density and velocity at time $t \geq 0$ and position $x \in \Omega_{\ell}$, respectively; $P$ is the pressure, smooth function of $\rho$, where for given $\rho_{*}$ positive number we assume $P^{\prime}\left(\rho_{*}\right)>0 ; \mu$ and $\mu^{\prime}$ are the viscosity

\footnotetext{
*Graduate School of Mathematics, Kyushu University, Fukuoka 819-0395, JAPAN

${ }^{\dagger}$ Faculty of Mathematics, Kyushu University, Fukuoka 819-0395, JAPAN
} 
coefficients that are assumed to be constants satisfying $\mu>0, \frac{2}{n} \mu+\mu^{\prime} \geq 0 ; \boldsymbol{g}$ is a timeperiodic external force of the form

$$
\boldsymbol{g}=\left(g^{1}\left(x_{n}, t\right), 0, \ldots, 0, g^{n}\left(x_{n}\right)\right)
$$

with $g^{1}$ being $\tau$-periodic function in time, where $\tau>0$.

The system (1.1)-(1.2) is considered under the boundary condition

$$
\left.v\right|_{x_{n}=0}=\left.v\right|_{x_{n}=\ell}=0,
$$

and the initial condition

$$
\left.(\rho, v)\right|_{t=0}=\left(\rho_{0}, v_{0}\right)
$$

Under suitable smallness conditions on $g^{n}$, problem (1.1)-(1.3) has a time-periodic solution $\bar{u}_{p}=\left(\bar{\rho}_{p}, \bar{v}_{p}\right)$

$$
\begin{gathered}
\bar{\rho}_{p}=\bar{\rho}_{p}\left(x_{n}\right), \\
\bar{v}_{p}=\left(\bar{v}_{p}^{1}\left(x_{n}, t\right), 0, \ldots, 0\right),
\end{gathered}
$$

where $\bar{\rho}_{p}$ and $\bar{v}_{p}$ satisfy

$$
\left|\bar{\rho}_{p}-\rho^{*}\right|_{C^{0}[0, \ell]} \leq C \frac{\mu\left|g^{n}\right|_{C^{0}[0, \ell]}}{P^{\prime}\left(\rho_{*}\right) \rho_{*} V}, \quad V=\frac{\rho_{*} \ell^{2}}{\mu}\left|g^{1}\right|_{C^{0}\left(\mathbb{R} ; L^{2}(0, \ell)\right)},
$$

and

$$
\left|\bar{v}_{p}^{1}\right|_{C^{0}\left(\mathbb{R} ; L^{2}[0, \ell]\right)} \leq C \frac{\rho_{*} \ell^{2}\left|g^{1}\right|_{C^{0}\left(\mathbb{R} ; L^{2}(0, \ell)\right)}}{\mu} .
$$

We are interested in large time behavior of solutions to the problem (1.1)-(1.4) when the initial value $\left(\rho_{0}, v_{0}\right)$ is sufficiently close to the value of time-periodic solution $\bar{u}_{p}=\left(\bar{\rho}_{p}, \bar{v}_{p}\right)$ at some fixed time. In this paper we deal with the linearized problem around the time-periodic flow.

Lately stability of stationary parallel flows for compressible Navier-Stokes equation has been investigated in $[2,3,4,5,6]$. It was proved in $[4,5]$, that stationary parallel flow is asymptotically stable for sufficiently small initial disturbances if the Reynolds number and Mach number are sufficiently small. Furthermore, when $n \geq 3$ the disturbances behave in large time as solutions of the linearized problem, whose asymptotic leading parts are given by solutions of an $n-1$ dimensional linear heat equation with convective term. On the other hand, when $n=2$ the asymptotic behavior is no longer described by the linearized problem; and it is described by a nonlinear diffusion equation, namely, by a 1-dimensional viscous Burgers equation.

In this paper we will extend previously obtained results for stationary parallel flows to the case of general time-periodic parallel flows. Combining techniques used in $[2,3,6]$ and [1] we will treat the linearized problem around time-periodic parallel flow and establish decay estimates on solutions similar to those in the case of the stationary parallel flows. Whereas $[2,3,6]$ are concerned with the stability of the stationary parallel flows, in [1] the diffusive 
stability of oscillations in reaction-diffusion systems is treated. Our main result in this paper reads as follows. We set $V=\frac{\rho_{*} \ell^{2}}{\mu}\left|g^{1}\right|_{\infty, 2}>0$ and introduce parameters:

$$
\nu=\frac{\mu}{\rho_{*} \ell V}, \quad \nu^{\prime}=\frac{\mu^{\prime}}{\rho_{*} \ell V}, \quad \gamma=\frac{\sqrt{P^{\prime}\left(\rho_{*}\right)}}{V},
$$

with $|\cdot|_{\infty, 2}$ being the norm in space $C^{0}\left(\mathbb{R} ; L^{2}(0,1)\right)$. We note that the Reynolds number $R e$ and Mach number $M a$ are given by $R e=\nu^{-1}$ and $M a=\gamma^{-1}$, respectively. After a suitable non-dimensionalisation, the linearized problem is written as follows:

$$
\begin{gathered}
\partial_{t} \phi+v_{p}^{1} \partial_{x_{1}} \phi+\gamma^{2} \operatorname{div}\left(\rho_{p} w\right)=0 \\
\partial_{t} w-\frac{\nu}{\rho_{p}} \Delta w-\frac{\nu+\nu^{\prime}}{\rho_{p}} \nabla \operatorname{div} w+v_{p}^{1} \partial_{x_{1}} w^{n}+\left(\partial_{x_{n}} v_{p}^{1}\right) w^{n} \boldsymbol{e}_{1} \\
+\frac{\nu}{\gamma^{2} \rho_{p}^{2}}\left(\partial_{x_{n}}^{2} v_{p}^{1}\right) \phi \boldsymbol{e}_{1}+\nabla\left(\frac{\widetilde{P}^{\prime}\left(\rho_{p}\right)}{\gamma^{2} \rho_{p}} \phi\right)=0 \\
\left.w\right|_{x_{n}=0}=\left.w\right|_{x_{n}=1}=0 \\
\left.(\phi, w)\right|_{t=0}=\left(\phi_{0}, w_{0}\right)
\end{gathered}
$$

in $\Omega$. Here $\phi=\phi(x, t)$ and $w=\left(w^{1}(x, t), \ldots, w^{n}(x, t)\right)$ denote the unknowns. The domain $\Omega_{l}$ is transformed into $\Omega=\mathbb{R}^{n-1} \times(0,1)$; and $\left(\bar{\rho}_{p}, \bar{v}_{p}\right)$ is transformed into $\left(\rho_{p}, v_{p}\right)$, where $v_{p}=\left(v_{p}^{1}\left(x_{n}, t\right), 0, \ldots, 0\right)$ is $T$-periodic in $t$ with $T=\frac{V}{\ell} \tau$. We write (1.5)-(1.8) in the form

$$
\partial_{t} u+L(t) u=\mathbf{0},\left.\quad w\right|_{x_{n}=0,1}=0,\left.\quad u\right|_{t=s}=u_{0},
$$

where $u={ }^{T}(\phi, w) ; L(t)$ is the linearized operator and $u_{0}={ }^{T}\left(\phi_{0}, w_{0}\right)$. We will prove that if $R e$ and $M a$ are sufficiently small, then the solution $u_{s}(t)=(\phi(t), w(t))$ of the linearized problem (1.9) satisfies

$$
\begin{aligned}
& \left\|\partial_{x^{\prime}}^{k} \partial_{x_{n}}^{l} u_{s}(t)\right\|_{2} \\
& \leq C\left\{(t-s)^{-\frac{n-1}{4}-\frac{k}{2}}\left\|u_{0}\right\|_{L^{1}\left(\mathbb{R}^{n-1} ; H^{1}(0,1) \times L^{2}(0,1)\right)}+e^{-d(t-s)}\left(\left\|u_{0}\right\|_{H^{1} \times L^{2}}+\left\|\partial_{x^{\prime}} w_{0}\right\|_{2}\right)\right\},
\end{aligned}
$$

and

$$
\begin{aligned}
& \left\|\partial_{x^{\prime}}^{k} \partial_{x_{n}}^{l}\left(u_{s}(t)-\sigma_{t, s} u^{(0)}(t)\right)\right\|_{2} \\
& \leq C\left\{(t-s)^{-\frac{n-1}{4}-\frac{1}{2}-\frac{k}{2}}\left\|u_{0}\right\|_{L^{1}\left(\mathbb{R}^{n-1} ; H^{1}(0,1) \times L^{2}(0,1)\right)}+e^{-d(t-s)}\left(\left\|u_{0}\right\|_{H^{1} \times L^{2}}+\left\|\partial_{x^{\prime}} w_{0}\right\|_{2}\right)\right\},
\end{aligned}
$$

for $t-s \geq 4 T, s \geq 0, k, l=0,1$, where $u^{(0)}(t)=u^{(0)}\left(x_{n}, t\right)$ is a function $T$-periodic in $t$ and $\sigma_{t, s}=\sigma_{t, s}\left(x^{\prime}\right)$ is a function whose Fourier transform in $x^{\prime}$ is given by

$$
\mathscr{F}\left(\sigma_{t, s}\right)=e^{-\left(i \kappa_{0} \xi_{1}+\kappa_{1}\left|\xi^{\prime}\right|^{2}\right)(t-s)}\left[\widehat{\phi}_{0}\left(\xi^{\prime}\right)\right]
$$

Here $\left[\widehat{\phi}_{0}\left(\xi^{\prime}\right)\right]$ is a quantity given by

$$
\left[\widehat{\phi}_{0}\left(\xi^{\prime}\right)\right]=\int_{0}^{1} \widehat{\phi}_{0}\left(\xi^{\prime}, x_{n}\right) d x_{n},
$$


with $\widehat{\phi}_{0}$ being the Fourier transform of $\phi_{0}$ in $x^{\prime}$ and $\kappa_{0}, \kappa_{1}$ are positive constants depending on $\rho_{*}, l, V, \mu, \mu^{\prime}$ and $P^{\prime}\left(\rho_{*}\right)$. Precise statement of the results will be given in Section 3 .

As in the case of the stationary parallel flows [6] these decay estimates as well as a decomposition argument in the proof will be useful for the nonlinear problem, which will be treated elsewhere.

To obtain decay estimates as in [6], we consider the Fourier transform of (1.9) in $x^{\prime} \in$ $\mathbb{R}^{n-1}$. That can be written as

$$
\frac{d}{d t} u+\widehat{L}_{\xi^{\prime}}(t) u=0,\left.\quad u\right|_{t=s}=u_{0}
$$

on $H^{1}(0,1) \times L^{2}(0,1)$. Here $\widehat{L}_{\xi^{\prime}}(t)$ is an operator on $H^{1}(0,1) \times L^{2}(0,1)$ with domain $D\left(\widehat{L}_{\xi^{\prime}}(t)\right)=H^{1}(0,1) \times\left(H^{2}(0,1) \cap H_{0}^{1}(0,1)\right)$ with a dual variable $\xi^{\prime} \in \mathbb{R}^{n-1}$. We denote $\widehat{U}_{\xi^{\prime}}(t, s)$ the solution operator for $(1.12)$. The operator $\widehat{U}_{\xi^{\prime}}(t, s)$ has different characters between cases $\left|\xi^{\prime}\right| \ll 1$ and $\left|\xi^{\prime}\right| \gg 1$. We thus decompose the solution operator $\mathscr{U}(t, s)$ associated with (1.9) into three parts: $\mathscr{U}(t, s)=\mathscr{F}^{-1}\left(\left.\widehat{U}_{\xi^{\prime}}(t, s)\right|_{\left|\xi^{\prime}\right| \leq r}\right)+\mathscr{F}^{-1}\left(\left.\widehat{U}_{\xi^{\prime}}(t, s)\right|_{r \leq\left|\xi^{\prime}\right| \leq R}\right)+$ $\mathscr{F}^{-1}\left(\left.\widehat{U}_{\xi^{\prime}}(t, s)\right|_{\left|\xi^{\prime}\right| \geq R}\right)$ for some $0<r \ll 1 \ll R$, where $\mathscr{F}^{-1}$ denotes the inverse Fourier transform. Since $\widehat{L}_{\xi^{\prime}}(t)$ is periodic in $t$, we investigate the monodromy operator $\widehat{U}_{\xi^{\prime}}(T)=\widehat{U}_{\xi^{\prime}}(T, 0)$ for $\left|\xi^{\prime}\right| \leq r \ll 1$ as in [1] and $\widehat{U}_{\xi^{\prime}}(T)$ can be regarded as a perturbation from $\widehat{U}_{0}(T)=$ $\left.\widehat{U}_{\xi^{\prime}}(T)\right|_{\xi^{\prime}=0}$. We will find that the spectrum of $\mathscr{U}(t, s)$ near 1 is given by that of monodromy operator $\widehat{U}_{\xi^{\prime}}(T)$ with $\left|\xi^{\prime}\right| \leq r \ll 1$, which is parameterized as $1-i \kappa_{0} \xi_{1} T-\kappa_{1}\left|\xi^{\prime}\right|^{2} T+O\left(\left|\xi^{\prime}\right|^{3}\right) T$ with some $\kappa_{0} \in \mathbb{R}, \kappa_{1}>0$, provided $R e$ and $M a$ are sufficiently small. On the other hand, if $\left|\xi^{\prime}\right| \geq R \gg 1$, we can derive the exponential decay property of the corresponding part of the solution operator $\mathscr{U}(t, s)$ by the Fourier transformed version of Matsumura-Nishida's energy method (see [3, 8]), provided that $R e$ and $M a$ are sufficiently small. As for the bounded frequency part $r \leq\left|\xi^{\prime}\right| \leq R$, we employ a certain time-dependent decomposition argument and apply a variant of Matsumura-Nishida's energy method as in [6] to show the exponential decay. As a result, one can see that the solution of the linearized problem behaves as $\sigma_{t, s}\left(x^{\prime}\right) u^{(0)}\left(x_{n}, t\right)=\mathscr{F}^{-1}\left(e^{-\left(i \kappa_{0} \xi_{1}+\kappa_{1}\left|\xi^{\prime}\right|^{2}\right)(t-s)}\left[\widehat{\phi}_{0}\left(\xi^{\prime}\right)\right]\right) u^{(0)}\left(x_{n}, t\right)$, provided that $R e$ and $M a$ are sufficiently small.

Problem (1.1)-(1.4) with $\boldsymbol{g}=\left(g^{1}\left(x_{n}, t\right), 0, \ldots, 0, g^{n}\left(x_{n}\right)\right)$ also covers another particularly interesting problem. Let us for a moment consider problem (1.1)-(1.2) together with $\boldsymbol{g}=$ $\left(0, \ldots, 0, g^{n}\left(x_{n}\right)\right)$ and boundary condition

$$
\left.v\right|_{x_{n}=0}=V^{1}(t) \boldsymbol{e}_{1},\left.\quad v\right|_{x_{n}=\ell}=0,
$$

where $V^{1}$ is $\tau$-periodic function of time and $\boldsymbol{e}_{1}=(1,0, \ldots, 0) \in \mathbb{R}^{n}$. This problem is a natural extension of Stokes' second problem from half space to infinite strip for compressible fluid. The motion of a fluid is caused by the periodic oscillation of a boundary plate. The study of the flow of a viscous fluid over an oscillating plate is not only of theoretical interest, but it also occurs in many applied problems and since Stokes (1851) it has received much attention under various settings. It is straightforward to see, that if we seek solution to this problem in the form $v=\left(1-x_{n}\right) V^{1}(t) \boldsymbol{e}_{1}+\tilde{v}$, then behavior of $\tilde{v}$ is governed by the same 
linearized problem as we get for (1.1)-(1.3) with $\boldsymbol{g}=\left(-\left(1-x_{n}\right) \partial_{t} V^{1}(t) / \rho, 0, \ldots, 0, g^{n}\left(x_{n}\right)\right)$ and therefore our result also holds for this particular problem.

This paper is organized as follows. In Section 2 we rewrite the problem into a nondimensional one and state the existence of time-periodic parallel flows. Our main results are stated in Section 3. In Section 4 we prove the exponential decay estimates. Finally, in Section 5 we prove the asymptotic behavior (1.10) and (1.11).

\section{Periodic Solution and the Linearized Problem}

In this section we state the existence of time-periodic solution and then we rewrite the problem into the one for the disturbance in a non-dimensional form. At the end of this section we introduce notation that will be used throughout this paper. Let $\rho_{*}$ be a given positive number. Throughout the paper we assume that

$$
P^{\prime}\left(\rho_{*}\right)>0 \text {. }
$$

We introduce the following dimensionless variables:

$$
x=\ell \widetilde{x}, \quad t=\frac{\ell}{V} \widetilde{t}, \quad v=V \widetilde{v}, \quad \rho=\rho_{*} \widetilde{\rho}, \quad P=\rho_{*} V^{2} \widetilde{P}, \quad \boldsymbol{g}\left(x_{n}, t\right)=\frac{\mu V}{\rho_{*} \ell^{2}} \widetilde{\boldsymbol{g}}\left(\widetilde{x}_{n}, \widetilde{t}\right),
$$

where

$$
V=\frac{\rho_{*} \ell^{2}}{\mu}\left|g_{1}\right|_{\infty, 2}
$$

Then the problem (1.1)-(1.4) is transformed into the following dimensionless problem on the layer $\Omega=\mathbb{R}^{n-1} \times(0,1)$ :

$$
\begin{gathered}
\partial_{t} \widetilde{\rho}+\operatorname{div}(\widetilde{\rho v})=0, \\
\widetilde{\rho}\left(\partial_{t} \widetilde{v}+(\widetilde{v} \cdot \nabla) \widetilde{v}\right)-\nu \Delta \widetilde{v}-\left(\nu+\nu^{\prime}\right) \nabla \operatorname{div} \widetilde{v}+\widetilde{P}^{\prime}(\widetilde{\rho}) \nabla \widetilde{\rho}=\nu \widetilde{\rho} \widetilde{g} \\
\left.\widetilde{v}\right|_{x_{n}=0}=\left.\widetilde{v}\right|_{x_{n}=1}=0, \\
\left.(\widetilde{\rho}, \widetilde{v})\right|_{t=0}=\left(\widetilde{\rho}_{0}, \widetilde{v}_{0}\right) .
\end{gathered}
$$

Here $\nu$ and $\nu^{\prime}$ are the non-dimensional parameters:

$$
\nu=\frac{\mu}{\rho_{*} \ell V}, \quad \nu^{\prime}=\frac{\mu^{\prime}}{\rho_{*} \ell V} .
$$

We also introduce parameters $\gamma$ and $T$ :

$$
\gamma=\sqrt{\widetilde{P}^{\prime}(1)}, \quad T=\frac{V}{\ell} \tau
$$

where $T$ is period of $\widetilde{g}_{1}$ in time. We note that the Reynolds number Re and Mach number $M a$ are given by $R e=\nu^{-1}$ and $M a=\gamma^{-1}$, respectively. In what follows we omit "tilde" of $\widetilde{x}_{n}$ and $\widetilde{t}$. 
One can see that if $\left|\widetilde{g}^{n}\right|_{\infty}$ is small enough then a time-periodic solution $\left(\rho_{p}, v_{p}\right)=$ $\left(\rho_{p}\left(x_{n}\right), v_{p}^{1}\left(x_{n}, t\right) \boldsymbol{e}_{1}\right)$ exists. More precisely, substituting $(\widetilde{\rho}, \widetilde{v})=\left(\rho_{p}\left(x_{n}\right), v_{p}^{1}\left(x_{n}, t\right) \boldsymbol{e}_{1}\right)$ into (2.1)-(2.3), we have

$$
\begin{gathered}
\partial_{t} v_{p}^{1}-\frac{\nu}{\rho_{p}} \partial_{x_{n}}^{2} v_{p}^{1}=\nu \widetilde{g}^{1} \\
\partial_{x_{n}}\left(\widetilde{P}\left(\rho_{p}\right)\right)=\nu \rho_{p} \widetilde{g}^{n} \\
\left.v_{p}^{1}\right|_{x_{n}=0}=\left.v_{p}^{1}\right|_{x_{n}=1}=0 .
\end{gathered}
$$

We state the existence of a time-periodic solution to (2.5)-(2.7) with

$$
1=\int_{0}^{1} \widetilde{\rho}\left(x_{n}\right) d x_{n}
$$

Proposition 2.1. Assume that $\widetilde{P}^{\prime}(\rho)>0$ for $\rho_{1} \leq \rho \leq \rho_{2}$ with some $0<\rho_{1}<1<\rho_{2}<2$. Let $\Phi(\rho)=\int_{1}^{\rho} \frac{\widetilde{P}^{\prime}(\eta)}{\eta} d \eta$ for $\rho_{1} \leq \rho \leq \rho_{2}$ and let $\Psi(r)=\Phi^{-1}(r)$ for $r_{1} \leq r \leq r_{2}$. Here $\Phi^{-1}$ denotes the inverse function of $\Phi$ and $r_{j}=\Phi\left(\rho_{j}\right)(j=1,2)$. If

$$
\nu\left|\widetilde{g}^{n}\right|_{\infty} \leq C \min \left\{\left|r_{1}\right|, r_{2}, \frac{1}{4 \gamma^{2}\left|\Psi^{\prime \prime}\right|_{C^{0}\left[r_{1}, r_{2}\right]}}\right\} \leq C,
$$

then there exists a smooth time-periodic solution $\left(\rho_{p}, v_{p}\right)=\left(\rho_{p}\left(x_{n}\right), v_{p}^{1}\left(x_{n}, t\right) \boldsymbol{e}_{1}\right)$ of (2.5)-(2.8) satisfying

$$
\begin{gathered}
\rho_{1} \leq \rho_{p}\left(x_{n}\right) \leq \rho_{2}, \quad\left|\rho_{p}-1\right|_{\infty} \leq C \frac{\nu}{\gamma^{2}}\left|\widetilde{g}^{n}\right|_{\infty}, \\
v_{p}^{1}\left(x_{n}, t\right)=\nu \int_{-\infty}^{t} e^{-\nu A(t-s)} \widetilde{g}^{1}\left(x_{n}, s\right) d s
\end{gathered}
$$

where $A$ denotes the uniformly elliptic operator on $L^{2}(0,1)$ with domain $D(A)=H^{2}(0,1) \cap$ $H_{0}^{1}(0,1)$ and $A v=-\frac{1}{\rho_{p}\left(x_{n}\right)} \partial_{x_{n}}^{2} v$ for $v \in D(A)$. Furthermore we have estimates

$$
\begin{gathered}
\left|\partial_{t}^{k} v_{p}^{1}\right|_{\infty, 2} \leq C\left|\partial_{t}^{k} \widetilde{g}^{1}\right|_{\infty, 2}, \quad k \geq 0, \\
\left|\partial_{x_{n}} v_{p}^{1}\right|_{\infty, 2} \leq C\left(\frac{1}{\nu}\left|\partial_{t} \widetilde{g}^{1}\right|_{\infty, 2}+\left|\widetilde{g}^{1}\right|_{\infty, 2}\right), \\
\left|\partial_{t}^{k} \partial_{x_{n}}^{2} v_{p}^{1}\right|_{\infty, 2} \leq C\left(\frac{1}{\nu}\left|\partial_{t}^{k+1} \widetilde{g}^{1}\right|_{\infty, 2}+\left|\partial_{t}^{k} \widetilde{g}^{1}\right|_{\infty, 2}\right), \quad k \geq 0 .
\end{gathered}
$$

Additionally, if $\nu\left|\widetilde{g}^{n}\right|_{C^{k-1}[0,1]} \leq \eta$, then

$$
\left|\partial_{x_{n}}^{k} \rho_{p}\right|_{\infty} \leq C_{k} \nu\left|\widetilde{g}^{n}\right|_{C^{k-1}[0,1]} \text { for } k=1,2, \ldots
$$


Here $C_{k}$ are positive constants depending on $k, \eta,|\Psi|_{C^{k}\left[r_{1}, r_{2}\right]}$ and $\rho_{2}$. In particular,

$$
\begin{gathered}
\left|\partial_{x_{n}} \rho_{p}\right|_{\infty} \leq C \frac{\nu}{\gamma^{2}}\left|\widetilde{g}^{n}\right|_{\infty} \\
\left|\widetilde{P}^{\prime}\left(\rho_{p}\right)-\gamma^{2}\right|_{\infty} \leq C\left|\widetilde{P}^{\prime \prime}\right|_{C^{0}\left[\rho_{1}, \rho_{2}\right]} \frac{\nu}{\gamma^{2}}\left|\widetilde{g}^{n}\right|_{\infty} .
\end{gathered}
$$

Remark. Operator $A$ satisfies estimates

$$
\begin{gathered}
\left|e^{-\nu A t} v\right|_{2} \leq C e^{-\frac{\nu}{2} t}|v|_{2} \\
\left|\partial_{x_{n}} e^{-\nu A t} v\right|_{2} \leq C \frac{1}{t^{\frac{1}{2}}} e^{-\frac{\nu}{2} t}|v|_{2},
\end{gathered}
$$

for some $C>0$ and all $t>0$.

Proof. Proof of existence and properties of $\rho_{p}$ is the same as in [4], if we substitute $\nu \widetilde{g}^{n}$ for $\widetilde{g}^{n}$, so we omit it. Once we obtained $\rho_{p}$ we easily get $v_{p}^{1}$ solution of $(2.5)$ and (2.7). To obtain estimates on $v_{p}^{1}$ we combine iteratively following relations:

$$
\begin{gathered}
\partial_{t} \int_{-\infty}^{t} e^{-\nu A(t-s)} f(s) d s=f(t)+\int_{-\infty}^{t} \partial_{t}\left[e^{-\nu A(t-s)} f(s)\right] d s \\
=f(t)-\int_{-\infty}^{t} \partial_{s}\left[e^{-\nu A(t-s)} f(s)\right] d s+\int_{-\infty}^{t} e^{-\nu A(t-s)} \partial_{s} f(s) d s=\int_{-\infty}^{t} e^{-\nu A(t-s)} \partial_{s} f(s) d s \\
\partial_{x_{n}}^{2} v_{p}^{1}=\frac{\rho_{p}}{\nu}\left(\partial_{t} v_{p}^{1}-\nu \widetilde{g}^{1}\right), \\
\left|\partial_{x_{n}} v_{p}^{1}(t)\right|_{L^{2}} \leq\left|v_{p}^{1}(t)\right|_{L^{2}}^{\frac{1}{2}}\left|\partial_{x_{n}}^{2} v_{p}^{1}(t)\right|_{L^{2}}^{\frac{1}{2}} .
\end{gathered}
$$

Setting $\widetilde{\rho}=\rho_{p}+\gamma^{-2} \phi$ and $\widetilde{v}=v_{p}+w$ in (2.1)-(2.4) and neglecting nonlinear terms of $u={ }^{T}(\phi, w)$, we arrive at the linearized problem:

$$
\begin{gathered}
\partial_{t} \phi+v_{p}^{1} \partial_{x_{1}} \phi+\gamma^{2} \operatorname{div}\left(\rho_{p} w\right)=0 \\
\partial_{t} w-\frac{\nu}{\rho_{p}} \Delta w-\frac{\nu+\nu^{\prime}}{\rho_{p}} \nabla \operatorname{div} w+v_{p}^{1} \partial_{x_{1}} w+\left(\partial_{x_{n}} v_{p}^{1}\right) w^{n} \boldsymbol{e}_{1} \\
+\frac{\nu}{\gamma^{2} \rho_{p}^{2}}\left(\partial_{x_{n}}^{2} v_{p}^{1}\right) \phi \boldsymbol{e}_{1}+\nabla\left(\frac{\widetilde{P}^{\prime}\left(\rho_{p}\right)}{\gamma^{2} \rho_{p}} \phi\right)=\mathbf{0}, \\
\left.w\right|_{x_{n}=0}=\left.w\right|_{x_{n}=1}=0, \\
\left.(\phi, w)\right|_{t=0}=\left(\phi_{0}, w_{0}\right) .
\end{gathered}
$$

Our main concern in this paper is the estimates of solutions to the problem (2.10)-(2.13).

In the remaining part of this section we introduce some notation which will be used throughout the paper. For a domain $N$ we denote by $L^{2}(N)$ the usual Lebesgue space on $N$ and its norm is denoted by $\|\cdot\|_{L^{2}(N)}$. Let $m$ be a nonnegative integer. $H^{m}(N)$ denotes the $m$-th order $L^{2}$ Sobolev space on $N$ with norm $\|\cdot\|_{H^{m}(N)} \cdot C_{0}^{m}(N)$ stands for the set of 
all $C^{m}$ functions which have compact support in $N$. We denote by $H_{0}^{1}(N)$ the completion of $C_{0}^{1}(N)$ in $H^{1}(N)$.

We simply denote by $L^{2}(N)$ (resp., $\left.H^{m}(N)\right)$ the set of all vector fields $w={ }^{T}\left(w^{1}, \ldots, w^{n}\right)$ on $N$ with $w^{j} \in L^{2}(N)$ (resp., $\left.H^{m}(N)\right), j=1, \ldots, n$, and its norm is also denoted by $\|\cdot\|_{L^{2}(N)}$ (resp., $\left.\|\cdot\|_{H^{m}(N)}\right)$. For $u={ }^{T}(\phi, w)$ with $\phi \in H^{k}(N)$ and $w={ }^{T}\left(w^{1}, \ldots, w^{n}\right) \in H^{m}(N)$, we define $\|u\|_{H^{k}(N) \times H^{m}(N)}$ by $\|u\|_{H^{k}(N) \times H^{m}(N)}=\|\phi\|_{H^{k}(N)}+\|w\|_{H^{m}(N)}$. When $k=m$, we simply write $\|u\|_{H^{k}(N) \times H^{k}(N)}=\|u\|_{H^{k}(N)}$.

In case $N=\Omega$ we abbreviate $L^{2}(\Omega)$ (resp., $\left.H^{m}(\Omega)\right)$ as $L^{2}$ (resp., $\left.H^{m}\right)$. In particular, the norm $\|\cdot\|_{L^{2}(\Omega)}=\|\cdot\|_{L^{2}}$ is denoted by $\|\cdot\|_{p}$.

In case $N=(0,1)$ we denote the norm of $L^{2}(0,1)$ by $|\cdot|_{2}$. The inner product of $L^{2}(0,1)$ is denoted by

$$
(f, g)=\int_{0}^{1} f\left(x_{n}\right) \overline{g\left(x_{n}\right)} d x_{n}, \quad f, g \in L^{2}(0,1) .
$$

Here $\bar{g}$ denotes the complex conjugate of $g$. For $u_{j}={ }^{T}\left(\phi_{j}, w_{j}\right) \in L^{2}(0,1)$ with $w_{j}=$ ${ }^{T}\left(w_{j}^{1}, \ldots, w_{j}^{n}\right)(j=1,2)$, we also define a weighted inner product $\left\langle u_{1}, u_{2}\right\rangle$ by

$$
\left\langle u_{1}, u_{2}\right\rangle=\int_{0}^{1} \phi_{1} \bar{\phi}_{2} \frac{\widetilde{P}^{\prime}\left(\rho_{p}\right)}{\gamma^{4} \rho_{p}} d x_{n}+\int_{0}^{1} w_{1} \bar{w}_{2} \rho_{p} d x_{n} .
$$

Furthermore, for $f \in L^{1}(0,1)$ we denote the mean value of $f$ in $(0,1)$ by $[f]$ :

$$
[f]=(f, 1)=\int_{0}^{1} f\left(x_{n}\right) d x_{n} .
$$

For $u={ }^{T}(\phi, w) \in L^{1}(0,1)$ with $w={ }^{T}\left(w^{1}, \ldots, w^{n}\right)$ we define $[u]$ by

$$
[u]=[\phi]+\left[w^{1}\right]+\cdots+\left[w^{n}\right] .
$$

The norm of $H^{m}(0,1)$ is denoted by $|\cdot|_{H^{m}}$.

We often write $x \in \Omega$ as

$$
x={ }^{T}\left(x^{\prime}, x_{n}\right), \quad x^{\prime}={ }^{T}\left(x_{1}, \ldots, x_{n-1}\right) \in \mathbb{R}^{n-1} .
$$

Partial derivatives of a function $u$ in $x, x^{\prime}, x_{n}$ and $t$ are denoted by $\partial_{x} u, \partial_{x^{\prime}} u, \partial_{x_{n}} u$ and $\partial_{t} u$, respectively. We also write higher order partial derivatives of $u$ in $x$ as $\partial_{x}^{k} u=\left(\partial_{x}^{\alpha} u ;|\alpha|=k\right)$.

We denote the $k \times k$ identity matrix by $I_{k}$. In particular, when $k=n+1$, we simply write $I$ for $I_{n+1}$. We also define $(n+1) \times(n+1)$ diagonal matrices $Q_{j}, Q^{\prime}$ and $\widetilde{Q}$ by

$$
Q_{j}=\operatorname{diag}(0, \ldots, 0, \underbrace{1}_{j-t h}, 0, \ldots, 0), \quad j=0,1, \ldots, n,
$$

and

$$
Q^{\prime}=\operatorname{diag}(0,1, \ldots, 1,0), \quad \widetilde{Q}=\operatorname{diag}(0,1, \ldots, 1) .
$$

We then have, for $u={ }^{T}(\phi, w) \in \mathbb{R}^{n+1}, w={ }^{T}\left(w^{1}, \ldots, w^{n}\right)={ }^{T}\left(w^{\prime}, w^{n}\right)$,

$$
Q_{0} u=\left(\begin{array}{c}
\phi \\
0
\end{array}\right), \quad Q_{j} u=\left(\begin{array}{c}
0 \\
w^{j} \\
0
\end{array}\right), \quad Q_{n} u=\left(\begin{array}{c}
0 \\
0 \\
w^{n}
\end{array}\right), \quad Q^{\prime} u=\left(\begin{array}{c}
0 \\
w^{\prime} \\
0
\end{array}\right), \quad \widetilde{Q} u=\left(\begin{array}{c}
0 \\
w
\end{array}\right) .
$$


We note that

$$
\left[Q_{0} u\right]=[\phi] \text { for } u={ }^{T}(\phi, w) .
$$

For a function $f=f\left(x^{\prime}\right)\left(x^{\prime} \in \mathbb{R}^{n-1}\right)$, we denote its Fourier transform by $\widehat{f}$ or $\mathscr{F} f$ :

$$
\widehat{f}\left(\xi^{\prime}\right)=(\mathscr{F} f)\left(\xi^{\prime}\right)=\int_{\mathbb{R}^{n-1}} f\left(x^{\prime}\right) e^{-i \xi^{\prime} \cdot x^{\prime}} d x^{\prime} .
$$

The inverse Fourier transform is denoted by $\mathscr{F}^{-1}$ :

$$
\left(\mathscr{F}^{-1} f\right)(x)=(2 \pi)^{-(n-1)} \int_{\mathbb{R}^{n-1}} f\left(\xi^{\prime}\right) e^{i \xi^{\prime} \cdot x^{\prime}} d \xi^{\prime} .
$$

We will denote the resolvent set of a closed operator $B$ by $\rho(B)$ and the spectrum of $B$ by $\sigma(B)$. For a bounded linear operator $B$ we denote the spectral radius of $B$ by $r(B)$. For $\Lambda \in \mathbb{R}$ and $\theta \in\left(\frac{\pi}{2}, \pi\right)$ we denote:

$$
\Sigma(\Lambda, \theta)=\{\lambda \in \mathbb{C} ;|\arg (\lambda-\Lambda)| \leq \theta\} .
$$

We denote the set of bounded linear operators from $X_{1}$ to $X_{2}$ by $L\left(X_{1}, X_{2}\right)$, and if $X_{1}=X_{2}$, we simply write $L\left(X_{1}\right)$ for $L\left(X_{1}, X_{1}\right)$. The operator norm is denoted by $|\cdot|_{L\left(X_{1}, X_{2}\right)}$.

\section{Main Results}

Let us consider the linearized problem

$$
\partial_{t} u+L(t) u=0,\left.\quad w\right|_{x_{n}=0,1}=0,\left.\quad u\right|_{t=s}=u_{0} .
$$

Here $u={ }^{T}(\phi, w)$ and $L(t)$ is the operator of the form

$$
\begin{aligned}
L(t) & =\left(\begin{array}{cc}
v_{p}^{1}(t) \partial_{x_{1}} & \gamma^{2} \operatorname{div}\left(\rho_{p} \cdot\right) \\
\nabla\left(\frac{\widetilde{P}^{\prime}\left(\rho_{p}\right)}{\gamma^{2} \rho_{p}} \cdot\right) & -\frac{\nu}{\rho_{p}} \Delta I_{n}-\frac{\nu+\nu^{\prime}}{\rho_{p}} \nabla \operatorname{div}
\end{array}\right) \\
& +\left(\begin{array}{cc}
0 & 0 \\
\frac{\nu}{\gamma^{2} \rho_{p}^{2}}\left(\partial_{x_{n}}^{2} v_{p}^{1}(t)\right) \boldsymbol{e}_{1} & v_{p}^{1}(t) \partial_{x_{1}} I_{n}+\left(\partial_{x_{n}} v_{p}^{1}(t)\right) \boldsymbol{e}_{1}^{T} \boldsymbol{e}_{n}
\end{array}\right) .
\end{aligned}
$$

We introduce the space $Z_{s}$ defined by

$$
\begin{aligned}
Z_{s}=\left\{u={ }^{T}(\phi, w) ; \phi\right. & \in C_{l o c}\left([s, \infty) ; H^{1}\right), \\
\partial_{x^{\prime}}^{\alpha^{\prime}} w & \in C_{l o c}\left([s, \infty) ; L^{2}\right) \cap L_{l o c}^{2}\left([s, \infty) ; H_{0}^{1}\right)\left(\left|\alpha^{\prime}\right| \leq 1\right), \\
w & \left.\in C_{l o c}\left((s, \infty) ; H_{0}^{1}\right)\right\},
\end{aligned}
$$

where the linearized problem can be uniquely solved. We will denote the solution operator for (3.1) by $\mathscr{U}(t, s)$.

Theorem 3.1. Let $s \geq 0$ be arbitrarily given. For any initial data $u_{0}={ }^{T}\left(\phi_{0}, w_{0}\right)$ satisfying $u_{0} \in H^{1} \times L^{2}$ with $\partial_{x^{\prime}} w_{0} \in L^{2}$ there exists a unique solution $u_{s}(t)=\mathscr{U}(t, s) u_{0}$ of $(3.1)$ in $Z_{s}$. 
Furthermore, $\mathscr{U}(t, s) u_{0}$ satisfies estimates

$$
\left\|\mathscr{U}(t, s) u_{0}\right\|_{2} \leq C\left\|u_{0}\right\|_{2}
$$

and

$$
\begin{aligned}
\left\|\partial_{x} Q_{0} \mathscr{U}(t, s) u_{0}\right\|_{2} & +\left\|\partial_{x^{\prime}} \widetilde{Q} \mathscr{U}(t, s) u_{0}\right\|_{2}+(t-s)^{\frac{1}{2}}\left\|\partial_{x_{n}} \widetilde{Q} \mathscr{U}(t, s) u_{0}\right\|_{2} \\
& \leq C\left\{\left\|u_{0}\right\|_{H^{1} \times L^{2}}+\left\|\partial_{x^{\prime}} w_{0}\right\|_{2}\right\},
\end{aligned}
$$

for $0<t-s \leq 4 T, s \geq 0$.

Proof. The uniqueness in $Z_{s}$ can be shown by an elementary energy method. As for the existence, it is not difficult to show the unique existence of solution $u \in C_{l o c}\left([0, \infty) ; H^{1}\right) \times$ $\left(C_{l o c}\left([0, \infty) ; H_{0}^{1}\right) \cap L_{l o c}^{2}\left([0, \infty) ; H^{2}\right)\right)$ for $u_{0} \in H^{1} \times H_{0}^{1}$. Existence for $u_{0} \in H^{1} \times L^{2}$ with $\partial_{x^{\prime}} w_{0} \in L^{2}$ then follows from an approximation argument by using Lemma 4.4 and 4.5 below. The proof of the estimate also follows from Lemma 4.4 below.

Theorem 3.2. There exist constants $\nu_{0}>0$ and $\gamma_{0}>0$ such that if $\nu \geq \nu_{0}$ and $\gamma^{2} /\left(2 \nu+\nu^{\prime}\right) \geq$ $\gamma_{0}^{2}$, then for any initial data $u_{0}={ }^{T}\left(\phi_{0}, w_{0}\right)$ satisfying $u_{0} \in\left(H^{1} \times L^{2}\right) \cap L^{1}\left(\mathbb{R}^{n-1} ; H^{1}(0,1) \times\right.$ $\left.L^{2}(0,1)\right)$ with $\partial_{x^{\prime}} w_{0} \in L^{2}$ the solution $u_{s}(t)=\mathscr{U}(t, s) u_{0}$ of problem (3.1) can be decomposed as

$$
\mathscr{U}(t, s) u_{0}=\mathscr{U}^{(0)}(t, s) u_{0}+\mathscr{U}^{(\infty)}(t, s) u_{0},
$$

where each term on the right-hand side has the following properties for $t-s \geq 4 T, s \geq 0$.

$$
\begin{gathered}
\left\|\partial_{x^{\prime}}^{k} \partial_{x_{n}}^{l} \mathscr{U}^{(0)}(t, s) u_{0}\right\|_{2} \leq C(t-s)^{-\frac{n-1}{4}-\frac{k}{2}}\left\|u_{0}\right\|_{L^{1}\left(\mathbb{R}^{n-1} ; H^{1}(0,1) \times L^{2}(0,1)\right)}, \\
\left\|\partial_{x^{\prime}}^{k} \partial_{x_{n}}^{l}\left(\mathscr{U}^{(0)}(t, s) u_{0}-\sigma_{t, s} u^{(0)}(t)\right)\right\|_{2} \leq C(t-s)^{-\frac{n-1}{4}-\frac{1}{2}-\frac{k}{2}}\left\|u_{0}\right\|_{L^{1}\left(\mathbb{R}^{n-1} ; H^{1}(0,1) \times L^{2}(0,1)\right)},
\end{gathered}
$$

$k, l=0,1$. Here

$$
\sigma_{t, s}=\mathscr{F}^{-1}\left(e^{-\left(i \kappa_{0} \xi_{1}+\kappa_{1}\left|\xi^{\prime}\right|^{2}\right)(t-s)}\left[\widehat{\phi}_{0}\right]\right),
$$

$u^{(0)}(t)=u^{(0)}\left(x_{n}, t\right)$ is some T-periodic function (see Lemma 4.6 below), and $\kappa_{0} \in \mathbb{R}, \kappa_{1}>0$ are some constants satisfying

$$
\kappa_{1}=\frac{\gamma^{2}}{\nu} K, K>0
$$

$$
\left\|\partial_{x}^{l} \mathscr{U}^{(\infty)}(t, s) u_{0}\right\|_{2} \leq C e^{-d(t-s)}\left(\left\|u_{0}\right\|_{H^{1} \times L^{2}}+\left\|\partial_{x^{\prime}} w_{0}\right\|_{2}\right),
$$

$l=0,1$, for some positive constant $d$.

Remark. In both Theorems 3.1 and 3.2 we assume following smoothness for external force and boundary data:

$$
\widetilde{g}^{n} \in C^{1}[0,1], \quad \widetilde{g}^{1} \in C^{2}\left(0, \infty: L^{2}(0,1)\right) .
$$

We combine estimates from Proposition 2.1 with basic assumption (2.9) on $\nu\left|\widetilde{g}^{n}\right|_{\infty}$ to get

$$
\left|\rho_{p}-1\right|_{\infty} \leq \frac{C}{\gamma^{2}}, \quad\left|\partial_{x_{n}} \rho_{p}\right|_{\infty} \leq \frac{C}{\gamma^{2}}, \quad\left|\widetilde{P}^{\prime}\left(\rho_{p}\right)-\gamma^{2}\right|_{\infty} \leq \frac{C}{\gamma^{2}}
$$


Furthermore, without loss of generality, we also assume that

$$
\left|\partial_{x_{n}}^{2} \rho_{p}\right|_{\infty} \leq \frac{C}{\gamma^{2}}
$$

holds true. Therefore, the smallness assumptions on $\widetilde{g}^{n}$ in Theorems 3.1 and 3.2 are expressed in terms of smallness of $\frac{1}{\gamma^{2}}$.

Again regarding to Proposition 2.1, in what follows we use bounds on $v_{p}^{1}$ as

$$
\left|\partial_{t}^{k} v_{p}^{1}\right|_{\infty, 2}+\left|\partial_{x_{n}} v_{p}^{1}\right|_{\infty, 2}+\left|\partial_{t}^{k} \partial_{x_{n}}^{2} v_{p}^{1}\right|_{\infty, 2} \leq C, \quad k \geq 0 .
$$

The decay rate (3.2) is the same one as that of an $n-1$ dimensional heat kernel. Our result shows that this is an optimal decay rate, and, in fact, estimate (3.3) shows that the asymptotic leading part of solutions is given by an $n-1$ dimensional heat kernel, which moves in $x_{1}$ direction with a constant speed, multiplied by time-periodic function.

A proof of Theorem 3.2 will be outlined in Section 4 and Section 5. In Section 4 we prove the exponential decay for the bounded frequency part and high frequency part. In Section 5 we prove the asymptotic estimates (3.2) and (3.3) for low frequency part.

\section{Proof of Theorem 3.2 - 1. Exponential decay esti- mates}

In this section we introduce decomposition of $\mathscr{U}(t, s)$ based on size of $\left|\xi^{\prime}\right|$ and prove that bounded frequency and high frequency parts decay exponentially. From now on we simply denote $\nu+\nu^{\prime}$ by $\widetilde{\nu}$ :

$$
\widetilde{\nu}=\nu+\nu^{\prime}
$$

To simplify further calculations we suppose

$$
\nu \geq 1, \quad \tilde{\nu} \geq 1, \quad \gamma \geq 1, \quad \frac{\gamma^{2}}{\nu+\tilde{\nu}} \geq 1
$$

To prove Theorem 3.2, we consider the Fourier transform of (3.1) in $x^{\prime}$ variable. The Fourier transform of (3.1) is written as

$$
\begin{gathered}
\partial_{t} \widehat{\phi}+i \xi_{1} v_{p}^{1} \widehat{\phi}+i \gamma^{2} \xi^{\prime} \cdot\left(\rho_{p} \widehat{w}^{\prime}\right)+\gamma^{2} \partial_{x_{n}}\left(\rho_{p} \widehat{w}^{n}\right)=0 \\
\partial_{t} \widehat{w}^{\prime}+\frac{\nu}{\rho_{p}}\left(\left|\xi^{\prime}\right|^{2}-\partial_{x_{n}}^{2}\right) \widehat{w}^{\prime}-i \frac{\widetilde{\nu}}{\rho_{p}} \xi^{\prime}\left(i \xi^{\prime} \cdot \widehat{w}^{\prime}+\partial_{x_{n}} \widehat{w}^{n}\right) \\
+i \xi^{\prime} \frac{\widetilde{P}^{\prime}\left(\rho_{p}\right)}{\gamma^{2} \rho_{p}} \widehat{\phi}+i \xi_{1} v_{p}^{1} \widehat{w}^{\prime}+\left(\partial_{x_{n}} v_{p}^{1}\right) \widehat{w}^{n} \boldsymbol{e}_{1}^{\prime}+\frac{\nu}{\gamma^{2} \rho_{p}^{2}}\left(\partial_{x_{n}}^{2} v_{p}^{1}\right) \widehat{\phi} \boldsymbol{e}_{1}^{\prime}=0 \\
\partial_{t} \widehat{w}^{n}+\frac{\nu}{\rho_{p}}\left(\left|\xi^{\prime}\right|^{2}-\partial_{x_{n}}^{2}\right) \widehat{w}^{n}-\frac{\widetilde{\nu}}{\rho_{p}} \partial_{x_{n}}\left(i \xi^{\prime} \cdot \widehat{w}^{\prime}+\partial_{x_{n}} \widehat{w}^{n}\right)+\partial_{x_{n}}\left(\frac{\widetilde{P}^{\prime}\left(\rho_{p}\right)}{\gamma^{2} \rho_{p}} \widehat{\phi}\right)+i \xi_{1} v_{p}^{1} \widehat{w}^{n}=0, \\
\left.\widehat{w}\right|_{x_{n}=0,1}=0
\end{gathered}
$$


for $t>s \geq 0$, and

$$
\left.\widehat{u}\right|_{t=s}=\widehat{u}_{0}={ }^{T}\left(\widehat{\phi}_{0}, \widehat{w}_{0}\right) .
$$

Here $\widehat{\phi}=\widehat{\phi}\left(\xi^{\prime}, x_{n}, t\right)$ and $\widehat{w}=\widehat{w}\left(\xi^{\prime}, x_{n}, t\right)$ are the Fourier transform of $\phi=\phi\left(x^{\prime}, x_{n}, t\right)$ and $w=w\left(x^{\prime}, x_{n}, t\right)$ in $x^{\prime} \in \mathbb{R}^{n-1}$ with $\xi^{\prime} \in \mathbb{R}^{n-1}$ being the dual variable and $\boldsymbol{e}_{1}^{\prime}=(1,0, \ldots, 0) \in$ $\mathbb{R}^{n-1}$. We thus arrive at the following problem

$$
\frac{d}{d t} u+\widehat{L}_{\xi^{\prime}}(t) u=0,\left.\quad u\right|_{t=s}=u_{0}
$$

on $X \equiv H^{1}(0,1) \times L^{2}(0,1)$. Here, for each $t, \widehat{L}_{\xi^{\prime}}(t)$ is an operator on $X$ with domain $D\left(\widehat{L}_{\xi^{\prime}}(t)\right)=D \equiv H^{1}(0,1) \times\left(H^{2}(0,1) \cap H_{0}^{1}(0,1)\right)$ with a parameter $\xi^{\prime} \in \mathbb{R}^{n-1}$. Here $u=$ ${ }^{T}\left(\phi\left(x_{n}, t\right), w\left(x_{n}, t\right)\right)\left(x_{n} \in[0,1], t \geq s \geq 0\right)$ and $\widehat{L}_{\xi^{\prime}}(t)$ is the operator of the form

$$
\widehat{L}_{\xi^{\prime}}(t)=\widehat{A}_{\xi^{\prime}}+\widehat{B}_{\xi^{\prime}}(t)+\widehat{C}_{0}(t)
$$

where

$$
\widehat{A}_{\xi^{\prime}}=\left(\begin{array}{ccc}
0 & 0 & 0 \\
0 & \nu \mathscr{A}_{\xi^{\prime}} I_{n-1}+\frac{\widetilde{\nu}}{\rho_{p}} \xi^{\prime T} \xi^{\prime} & -i \frac{\widetilde{\nu}}{\rho_{p}} \xi^{\prime} \partial_{x_{n}} \\
0 & -i \frac{\widetilde{\nu} T}{\rho_{p}} \xi^{\prime} \partial_{x_{n}} & \nu \mathscr{A}_{\xi^{\prime}}-\frac{\widetilde{\nu}}{\rho_{p}} \partial_{x_{n}}^{2}
\end{array}\right)
$$

with

$$
\begin{gathered}
\mathscr{A}_{\xi^{\prime}}=\frac{1}{\rho_{p}}\left(\left|\xi^{\prime}\right|^{2}-\partial_{x_{n}}^{2}\right), \\
\widehat{B}_{\xi^{\prime}}(t)=\left(\begin{array}{ccc}
i \xi_{1} v_{p}^{1}(t) & i \gamma^{2} \rho_{p}^{T} \xi^{\prime} & \gamma^{2} \partial_{x_{n}}\left(\rho_{p} \cdot\right) \\
i \xi^{\prime} \frac{\widetilde{P}^{\prime}\left(\rho_{p}\right)}{\gamma^{2} \rho_{p}} & i \xi_{1} v_{p}^{1}(t) I_{n-1} & 0 \\
\partial_{x_{n}}\left(\frac{\widetilde{P}^{\prime}\left(\rho_{p}\right)}{\gamma^{2} \rho_{p}} \cdot\right) & 0 & i \xi_{1} v_{p}^{1}(t)
\end{array}\right), \\
\widehat{C}_{0}(t)=\left(\begin{array}{ccc}
0 & 0 & 0 \\
\frac{\nu}{\gamma^{2} \rho_{p}^{2}}\left(\partial_{x_{n}}^{2} v_{p}^{1}(t)\right) \boldsymbol{e}_{1}^{\prime} & 0 & \left(\partial_{x_{n}} v_{p}^{1}(t)\right) \boldsymbol{e}_{1}^{\prime} \\
0 & 0 & 0
\end{array}\right)
\end{gathered}
$$

For each fixed $\xi^{\prime}, \widehat{A}_{\xi^{\prime}}+\widehat{B}_{0}$ is a closed operator on $X$ with domain $D\left(\widehat{A}_{\xi^{\prime}}+\widehat{B}_{0}\right)=D$; and $-\left(\widehat{A}_{\xi^{\prime}}+\widehat{B}_{0}\right)$ generates an analytic semigroup in $X$ (see [2]). Since $v_{p}^{1}$ is bounded smooth function of $x_{n}, t, \widehat{L}_{\xi^{\prime}}(t)$ can be seen as a lower order perturbation of $\widehat{A}_{\xi^{\prime}}+\widehat{B}_{0}$. Therefore, we can show that

$$
(|\lambda|+1)\left|\left(\lambda+\widehat{L}_{\xi^{\prime}}(t)\right)^{-1}\right|_{L(X)} \leq C
$$

and

$$
\left|\left(\widehat{L}_{\xi^{\prime}}(t)-\widehat{L}_{\xi^{\prime}}(s)\right)\left(\Lambda+\widehat{L}_{\xi^{\prime}}(0)\right)^{-1}\right|_{L(X)} \leq C|t-s|^{\alpha},
$$

where $\lambda \in \Sigma(\Lambda, \theta)$ with some $\Lambda>0, \theta \in\left(\frac{\pi}{2}, \pi\right)$ and $\alpha>0$. It then follows from [9, Theorem 5.2.1] that, for each $\xi^{\prime} \in \mathbb{R}^{n-1}$, there exists a unique solution $\widehat{u}_{s}(t)=\widehat{U}_{\xi^{\prime}}(t, s) \widehat{u}_{0}$ of problem 
(4.1)-(4.5) satisfying $\widehat{u}_{s} \in C_{l o c}([s, \infty) ; X) \cap C^{1}((s, \infty) ; X) \cap C((s, \infty) ; D)$. Moreover, $\widehat{U}_{\xi^{\prime}}(t, s)$ satisfies $\left|\widehat{U}_{\xi^{\prime}}(t, s)\right|_{L(X)} \leq C_{t_{1}}$ and $\left|\widehat{L}_{\xi^{\prime}}(t) \widehat{U}_{\xi^{\prime}}(t, s)\right|_{L(X)} \leq C_{t_{1}}(t-s)^{-1}$ with $0 \leq s \leq t \leq t_{1}$ for all $t_{1} \geq 0$, which implies that $\left|\partial_{x_{n}} \widetilde{Q} \widehat{U}_{\xi^{\prime}}(t, s) u_{0}\right|_{2} \leq C_{t_{1}}(t-s)^{-\frac{1}{2}}\left|u_{0}\right|_{H^{1} \times L^{2}}$. The solution $\mathscr{U}(t, s) u_{0}$ of $(3.1)$ is then given by $\mathscr{U}(t, s) u_{0}=\mathscr{F}^{-1}\left(\widehat{U}_{\xi^{\prime}}(t, s) \widehat{u}_{0}\right)$ for all $t-s \geq 0, s \geq 0$.

We decompose $\mathscr{U}(t, s) u_{0}$ in the following way. Let $0<r<R$. Define $\chi^{(0)}\left(\xi^{\prime}\right), \chi^{(1)}\left(\xi^{\prime}\right)$ and $\chi^{(\infty)}\left(\xi^{\prime}\right)$ by

$$
\begin{gathered}
\chi^{(0)}\left(\xi^{\prime}\right)=1 \text { if }\left|\xi^{\prime}\right| \leq r, \chi^{(0)}\left(\xi^{\prime}\right)=0 \text { if }\left|\xi^{\prime}\right|>r, \\
\chi^{(\infty)}\left(\xi^{\prime}\right)=1 \text { if }\left|\xi^{\prime}\right| \geq R, \chi^{(\infty)}\left(\xi^{\prime}\right)=0 \text { if }\left|\xi^{\prime}\right|<R,
\end{gathered}
$$

and

$$
\chi^{(1)}=\left(1-\chi^{(0)}\right)\left(1-\chi^{(\infty)}\right)
$$

We decompose $\mathscr{U}(t, s) u_{0}$ as

$$
\mathscr{U}(t, s) u_{0}=\mathscr{V}_{0}(t, s) u_{0}+\mathscr{V}_{1}(t, s) u_{0}+\mathscr{V}_{\infty}(t, s) u_{0}
$$

where

$$
\mathscr{V}_{j}(t, s) u_{0}=\mathscr{F}^{-1}\left(\chi^{(j)} \widehat{U}_{\xi^{\prime}}(t, s) \widehat{u}_{0}\right), \quad j=0,1, \infty .
$$

In this section we will show that $\mathscr{V}_{j}(t, s) u_{0}(j=1, \infty)$ decay exponentially in time. The low frequency part $\mathscr{V}_{0}(t, s) u_{0}$ will be investigated in Section 5 , which all together gives estimates (3.2) and (3.3).

Proposition 4.1. There exist constants $R_{0}>1, \nu_{0}>0$ and $\gamma_{0}>0$ such that if $\nu \geq \nu_{0}$ and $\gamma^{2} /(\nu+\widetilde{\nu}) \geq \gamma_{0}^{2}$ then there exists a constant $d>0$ such that the estimate

$$
\left\|\mathscr{V}_{\infty}(t, s) u_{0}\right\|_{H^{1}} \leq C e^{-d(t-s-4 T)}\left(\left\|u_{0}\right\|_{H^{1} \times L^{2}}+\left\|\partial_{x^{\prime}} w_{0}\right\|_{2}\right)
$$

holds uniformly in $t-s \geq 4 T, s \geq 0$.

Proposition 4.2. There exist constants $\nu_{0}>0$ and $\gamma_{0}>0$ such that if $\nu \geq \nu_{0}$ and $\gamma^{2} /(\nu+\widetilde{\nu}) \geq \gamma_{0}^{2}$ then for any $0<r<R_{0}$ there exists a constant $d(r)>0$ such that the estimate

$$
\left\|\mathscr{V}_{1}(t, s) u_{0}\right\|_{H^{1}} \leq C e^{-d(t-s-4 T)}\left(\left\|u_{0}\right\|_{H^{1} \times L^{2}}+\left\|\partial_{x^{\prime}} w_{0}\right\|_{2}\right),
$$

holds uniformly in $t-s \geq 4 T, s \geq 0$.

Proof of Proposition 4.1. We investigate problem (4.6) for $\left|\xi^{\prime}\right| \geq R>0$ and prove Proposition 4.1. We also give a proof of the estimate given in Theorem 3.1.

We introduce following notation. For $v={ }^{T}(\phi, w)$, or $v=w$ we define $D_{\xi^{\prime}}(v)$ by

$$
D_{\xi^{\prime}}(v)=\left|\xi^{\prime}\right|^{2}|v|_{2}^{2}+\left|\partial_{x_{n}} v\right|_{2}^{2}
$$

First, we state the exponential decay estimate for large $t-s$. 
Lemma 4.3. There exist constants $R_{0}>1, \nu_{0}>0$ and $\gamma_{0}>0$ such that if $\nu \geq \nu_{0}$ and $\gamma^{2} /(\nu+\widetilde{\nu}) \geq \gamma_{0}^{2}$ then there exists a constant $d>0$ such that the estimate

$$
|\widehat{\phi}(t)|_{2}^{2}+|\widehat{w}(t)|_{2}^{2}+D_{\xi^{\prime}}\left(\widehat{u}_{s}\right)(t) \leq C e^{-d(t-s-4 T)}\left\{|\widehat{\phi}(4 T)|_{2}^{2}+|\widehat{w}(4 T)|_{2}^{2}+D_{\xi^{\prime}}\left(\widehat{u}_{s}\right)(4 T)\right\},
$$

holds uniformly for $t-s \geq 4 T, s \geq 0$ provided that $\left|\xi^{\prime}\right| \geq R_{0}$.

Proof of Lemma 4.3 is similar to the proof of [3, Proposition 6.1]. In our case in calculations appear new disturbance terms thanks to the $\rho_{p} \not \equiv 1$ and term $\frac{\nu}{\gamma^{2} \rho_{p}^{2}}\left(\partial_{x_{n}}^{2} v_{p}^{1}\right) \widehat{\phi} \boldsymbol{e}_{1}^{\prime}$, but those can be handled in standard way by smallness assumptions on Mach and Reynolds numbers, so we omit the proof. See also [4].

Second, we state estimates for $t-s$ near 0 .

Lemma 4.4. There holds the following estimate uniformly for $0<t-s \leq 4 T, s \geq 0$ and $\xi^{\prime} \in \mathbf{R}^{n-1}$ :

$$
\left(1+\left|\xi^{\prime}\right|^{2}\right)\left(|\widehat{\phi}(t)|_{2}^{2}+|\widehat{w}(t)|_{2}^{2}\right)+\left|\partial_{x_{n}} \widehat{\phi}(t)\right|_{2}^{2}+(t-s)\left|\partial_{x_{n}} \widehat{w}(t)\right|_{2}^{2} \leq C\left\{\left(1+\left|\xi^{\prime}\right|^{2}\right)\left|\widehat{u}_{0}\right|_{2}^{2}+\left|\partial_{x_{n}} \widehat{\phi}_{0}\right|_{2}^{2}\right\} \text {. }
$$

Proposition 4.1 is an immediate consequence of Lemma 4.3 and 4.4.

The estimate in Theorem 3.1 also follows from Lemma 4.4. It remains to prove Lemma 4.4. To prove Lemma 4.4 we employ the following estimates.

Lemma 4.5. For all $0 \leq t-s \leq 4 T, s \geq 0$ there hold the estimates

$$
\left(1+\left|\xi^{\prime}\right|^{2}\right)\left(\frac{1}{\gamma^{2}}|\widehat{\phi}(t)|_{2}^{2}+|\widehat{w}(t)|_{2}^{2}\right)+\nu \int_{s}^{t}\left(1+\left|\xi^{\prime}\right|^{2}\right) D_{\xi^{\prime}}(\widehat{w}) d s \leq C\left(1+\left|\xi^{\prime}\right|^{2}\right)\left(\frac{1}{\gamma^{2}}\left|\widehat{\phi}_{0}\right|_{2}^{2}+\left|\widehat{w}_{0}\right|_{2}^{2}\right)
$$

and

$$
\left|\partial_{x_{n}} \widehat{\phi}(t)\right|_{2}^{2} \leq C\left\{\left(1+\left|\xi^{\prime}\right|^{2}\right)\left|\widehat{u}_{0}\right|_{2}^{2}+\left|\partial_{x_{n}} \widehat{\phi}_{0}\right|_{2}^{2}\right\}
$$

Proof of Lemma 4.5 is similar to the proof of [3, Proposition 6.11], so we omit the proof.

Proof of Lemma 4.4. We write (4.2) and (4.3) as

$$
\partial_{t} w+\widehat{T}_{\xi^{\prime}} w=-\widehat{E}_{\xi^{\prime}}(t) w-\widehat{F}_{\xi^{\prime}}(t) \phi .
$$

Here $\widehat{T}_{\xi^{\prime}}$ is the operator on $L^{2}(0,1)$ of the form

$$
\widehat{T}_{\xi^{\prime}}=\left(\begin{array}{cc}
\frac{\nu}{\rho_{p}}\left(\left|\xi^{\prime}\right|^{2}-\partial_{x_{n}}^{2}\right) I_{n-1}+\frac{\widetilde{\nu}}{\rho_{p}} \xi^{T} \xi^{\prime} & -i \frac{\widetilde{\nu}}{\rho_{p}} \xi^{\prime} \partial_{x_{n}} \\
-i \frac{\widetilde{\nu}}{\rho_{p}} T \xi^{\prime} \partial_{x_{n}} & \frac{\nu}{\rho_{p}}\left(\left|\xi^{\prime}\right|^{2}-\partial_{x_{n}}^{2}\right)-\frac{\widetilde{\nu}}{\rho_{p}} \partial_{x_{n}}^{2}
\end{array}\right),
$$

with domain $D\left(\widehat{T}_{\xi^{\prime}}\right)=H^{2}(0,1) \cap H_{0}^{1}(0,1)$,

$$
\widehat{E}_{\xi^{\prime}}(t)=\left(\begin{array}{cc}
i \xi_{1} v_{p}^{1}(t) I_{n-1} & \left(\partial_{x_{n}} v_{p}^{1}(t)\right) \boldsymbol{e}_{1}^{\prime} \\
0 & i \xi_{1} v_{p}^{1}(t)
\end{array}\right)
$$


and

$$
\widehat{F}_{\xi^{\prime}}(t)=\left(\begin{array}{c}
i \xi^{\prime} \frac{\widetilde{P}^{\prime}\left(\rho_{p}\right)}{\gamma^{2} \rho_{p}}+\frac{\nu}{\gamma^{2} \rho_{p}^{2}}\left(\partial_{x_{n}}^{2} v_{p}^{1}(t)\right) \boldsymbol{e}_{1}^{\prime} \\
\partial_{x_{n}}\left(\frac{\widetilde{P}^{\prime}\left(\rho_{p}\right)}{\gamma^{2} \rho_{p}} \cdot\right)
\end{array}\right)
$$

Then $w$ is written as

$$
w(t)=e^{-(t-s) \widehat{T}_{\xi^{\prime}}} w_{0}-\int_{s}^{t} e^{-(t-z) \widehat{T}_{\xi^{\prime}}} \widehat{E}_{\xi^{\prime}}(z) w(z) d z-\int_{s}^{t} e^{-(t-z) \widehat{T}_{\xi^{\prime}}} \widehat{F}_{\xi^{\prime}}(z) \phi(z) d z .
$$

Using the equality

$$
\left(\widehat{T}_{\xi^{\prime}} w, \rho_{p} w\right)=\nu D_{\xi^{\prime}}(w)+\widetilde{\nu}\left|i \xi^{\prime} \cdot w^{\prime}+\partial_{x_{n}} w^{n}\right|_{2}^{2}
$$

one can see that

$$
\left|\partial_{x_{n}}^{l} e^{-t \widehat{T}_{\xi^{\prime}}} w_{0}\right|_{2} \leq C t^{-\frac{l}{2}}\left|w_{0}\right|_{2} \quad(l=0,1)
$$

for $0<t \leq 4 T$.

We have estimate

$$
\left|\widehat{E}_{\xi^{\prime}}(z) w(z)\right|_{2} \leq C\left(1+\left|\xi^{\prime}\right|\right)|w(z)|_{2} \leq C\left(1+\left|\xi^{\prime}\right|\right)\left|u_{0}\right|_{2}
$$

and moreover by (4.7) and (4.8) we have

$$
\begin{aligned}
\left|\widehat{F}_{\xi^{\prime}}(z) \phi(z)\right|_{2} & \leq C\left\{\left(1+\left|\xi^{\prime}\right|\right)|\phi(z)|_{2}+\left|\partial_{x_{n}} \phi(z)\right|_{2}\right\} \\
& \leq C\left\{\left(1+\left|\xi^{\prime}\right|\right)\left|u_{0}\right|_{2}+\left|\partial_{x_{n}} \phi_{0}\right|_{2}\right\}
\end{aligned}
$$

It follows from (4.9)-(4.12) that

$$
\begin{aligned}
(t-s)^{\frac{1}{2}}\left|\partial_{x_{n}} w(t)\right|_{2} & \leq C\left|w_{0}\right|_{2}+C(t-s)^{\frac{1}{2}} \int_{s}^{t}(t-z)^{-\frac{1}{2}}\left(\left|\widehat{E}_{\xi^{\prime}}(z) w(z)\right|_{2}+\left|\widehat{F}_{\xi^{\prime}}(z) \phi(z)\right|_{2}\right) d z \\
& \leq C\left|w_{0}\right|_{2}+C\left\{\left(1+\left|\xi^{\prime}\right|\right)\left|u_{0}\right|_{2}+\left|\partial_{x_{n}} \phi_{0}\right|_{2}\right\}
\end{aligned}
$$

for $0<t-s \leq 4 T$. This, together with (4.7) and (4.8) gives the desired estimate of Lemma 4.4 .

We next prove Proposition 4.2. To prove Proposition 4.2 we decompose $\chi^{(1)} \widehat{U}_{\xi^{\prime}}(t, s) \widehat{u}_{0}$ based on a spectral property of $\widehat{L}_{\xi^{\prime}}(t)$ with $\xi^{\prime}=0$, namely, $\widehat{L}_{0}(t)$.

We introduce the formal adjoint operator of $\widehat{L}_{\xi^{\prime}}(t)$ with respect to the weighted inner product $\langle\cdot, \cdot\rangle$. We define an operator $\widehat{L}_{\xi^{\prime}}^{*}(t)$ by

$$
\widehat{L}_{\xi^{\prime}}^{*}(t)=\widehat{A}_{\xi^{\prime}}^{*}+\widehat{B}_{\xi^{\prime}}^{*}(t)+\widehat{C}_{0}^{*}(t)
$$

with domain of definition $D\left(\widehat{L}_{\xi^{\prime}}^{*}(t)\right)=D$, where

$$
\widehat{A}_{\xi^{\prime}}^{*}=\widehat{A}_{\xi^{\prime}}, \quad \widehat{B}_{\xi^{\prime}}^{*}(t)=-\widehat{B}_{\xi^{\prime}}(t),
$$


and

We then have

$$
\widehat{C}_{0}^{*}(t)=\left(\begin{array}{ccc}
0 & \frac{\nu}{\gamma^{2} \rho_{p}^{2}}\left(\partial_{x_{n}}^{2} v_{p}^{1}\right)^{T} \boldsymbol{e}_{1}^{\prime} & 0 \\
0 & 0 & 0 \\
0 & \left(\partial_{x_{n}} v_{p}^{1}\right)^{T} \boldsymbol{e}_{1}^{\prime} & 0
\end{array}\right)
$$

$$
\begin{gathered}
\left\langle\widehat{A}_{\xi^{\prime}} u, v\right\rangle=\left\langle u, \widehat{A}_{\xi^{\prime}}^{*} v\right\rangle=\left\langle u, \widehat{A}_{\xi^{\prime}} v\right\rangle, \\
\left\langle\widehat{B}_{\xi^{\prime}}(t) u, v\right\rangle=\left\langle u, \widehat{B}_{\xi^{\prime}}^{*}(t) v\right\rangle=-\left\langle u, \widehat{B}_{\xi^{\prime}}(t) v\right\rangle, \\
\left\langle\widehat{C}_{0}(t) u, v\right\rangle=\left\langle u, \widehat{C}_{0}^{*}(t) v\right\rangle,
\end{gathered}
$$

and

$$
\left\langle\widehat{L}_{\xi^{\prime}}(t) u, v\right\rangle=\left\langle u, \widehat{L}_{\xi^{\prime}}^{*}(t) v\right\rangle
$$

for $u, v \in D$.

\section{Lemma 4.6.}

(i) There exists a T-time-periodic solution $u^{(0)}\left(x_{n}, t\right)$ of

$$
\begin{gathered}
\partial_{t} u+\widehat{L}_{0}(t) u=0, \\
\left.w\right|_{x_{n}=0,1}=0,
\end{gathered}
$$

with following properties:

$$
u^{(0)}\left(x_{n}, t\right)={ }^{T}\left(\phi^{(0)}, \frac{1}{\gamma^{2}} w^{(0), 1}\left(x_{n}, t\right) \boldsymbol{e}_{1}^{\prime}, 0\right),
$$

where

$$
\begin{gathered}
\phi^{(0)}\left(x_{n}\right)=\alpha_{0} \frac{\gamma^{2} \rho_{p}\left(x_{n}\right)}{\widetilde{P}^{\prime}\left(\rho_{p}\left(x_{n}\right)\right)}, \quad \alpha_{0}=\left[\frac{\gamma^{2} \rho_{p}}{\widetilde{P}^{\prime}\left(\rho_{p}\right)}\right]^{-1}, \\
w^{(0), 1}\left(x_{n}, t\right)=-\int_{-\infty}^{t} e^{-(t-s) \nu A} \nu \frac{\alpha_{0} \gamma^{2}}{\widetilde{P}^{\prime}\left(\rho_{p}\right) \rho_{p}}\left(\partial_{x_{n}}^{2} v_{p}^{1}(s)\right) d s .
\end{gathered}
$$

We also have following estimates

$$
\left|\partial_{t}^{k} w^{(0), 1}(t)\right|_{L^{2}} \leq C\left|\partial_{t}^{k} \partial_{x_{n}}^{2} v_{p}^{1}(t)\right|_{L^{2}}, \quad k \geq 0 .
$$

(ii) Let $u_{0} \in X$. Then there holds

$$
\left[Q_{0} \widehat{U}_{0}(t, s) u_{0}\right]=\left[Q_{0} u_{0}\right], \text { for all } t \geq s \geq 0,
$$

where $\widehat{U}_{0}(t, s)$ denotes the solution operator for (4.13) and (4.14).

Proof. Fact that $u^{(0)}\left(x_{n}, t\right)$ is T-time-periodic solution can be shown by direct computation. Estimates on $\partial_{t}^{k} w^{(0), 1}$ are obtained in similar way as those for $v_{p}^{1}$ in from Proposition 2.1. Let $\widehat{U}_{0}(t, s) u_{0}={ }^{T}(\phi(t), w(t))$. The first row of equation (4.13) is written as

$$
\begin{gathered}
\partial_{t} \phi+\gamma^{2} \partial_{x_{n}}\left(\rho_{p} w^{n}\right)=0 \\
16
\end{gathered}
$$


Taking mean value of this over $(0,1)$ we get

$$
\partial_{t}[\phi]+\gamma^{2}\left[\partial_{x_{n}}\left(\rho_{p} w^{n}\right)\right]=0 .
$$

Using integration by parts and boundary condition (4.14) we get $\left[\partial_{x_{n}}\left(\rho_{p} w^{n}\right)\right]=0$, and hence,

$$
\partial_{t}[\phi]=0 .
$$

This gives $[\phi(t)]=\left[\phi_{0}\right]$ for all $t \geq 0$.

\section{Definition 4.7.}

(i) Let us define projections $\widehat{\Pi}^{(0)}(t)$ and $\widehat{\Pi}^{(0) *}(t)$ as

$$
\begin{gathered}
\widehat{\Pi}^{(0)}(t) u=\left\langle u, u^{(0) *}\right\rangle u^{(0)}(t)=\left[Q_{0} u\right] u^{(0)}(t), \\
\widehat{\Pi}^{(0) *}(t) u=\left\langle u, u^{(0)}(t)\right\rangle u^{(0) *}
\end{gathered}
$$

respectively, with

$$
u^{(0) *}={ }^{T}\left(\frac{\gamma^{2}}{\alpha_{0}} \phi^{(0)}, 0,0\right)
$$

We also define $\widehat{\Pi}_{c}^{(0)}(t)$ by

$$
\widehat{\Pi}_{c}^{(0)}(t)=I-\widehat{\Pi}^{(0)}(t)
$$

(ii) Let $u^{(0)}(t)=u_{0}^{(0)}+u_{1}^{(0)}(t)$, where

$$
u_{0}^{(0)}={ }^{T}\left(\phi^{(0)}, 0,0\right), \quad u_{1}^{(0)}(t)={ }^{T}\left(0, \frac{1}{\gamma^{2}} w^{(0), 1}(t) \boldsymbol{e}_{1}^{\prime}, 0\right) .
$$

We make a simple observation which will be useful in the argument below.

Lemma 4.8. Let $u \in X$.

(i) $\frac{d}{d t} Q_{0} \widehat{\Pi}^{(0)}(t) u=0$, i.e., $\phi$-component of $\widehat{\Pi}^{(0)}(t) u$ is independent of $t$.

(ii) Let $u_{1}(t)={ }^{T}\left(\phi_{1}, w_{1}(t)\right)=\widehat{\Pi}_{c}^{(0)}(t) u$. Then

$$
\left|\phi_{1}\right|_{2} \leq\left|\partial_{x_{n}} \phi_{1}\right|_{2}
$$

Furthermore, if $\widetilde{Q} u \in H_{0}^{1}(0,1)$ then

$$
\left|w_{1}(t)\right|_{2} \leq\left|\partial_{x_{n}} w_{1}(t)\right|_{2}
$$

Proof. It is easy to see (i). As for (ii), since $\left[\phi_{1}\right]=0$, the Poincaré inequality gives $\left|\phi_{1}\right|_{2} \leq$ $\left|\partial_{x_{n}} \phi_{1}\right|_{2}$. Let $\widetilde{Q} u \in H_{0}^{1}(0,1)$. Since $w^{(0), 1}(t) \in H_{0}^{1}(0,1)$ for each $t$, we have $\widetilde{Q} \widehat{\Pi}^{(0)}(t) u \in$ $H_{0}^{1}(0,1)$. Therefore, $w_{1}(t) \in H_{0}^{1}(0,1)$ for each $t$, and hence, the Poincaré inequality gives $\left|w_{1}(t)\right|_{2} \leq\left|\partial_{x_{n}} w_{1}(t)\right|_{2}$ for each $t$. This concludes the proof.

Based on Definition 4.7 for each $t$ we decompose $u(t)$ into the parts of $\widehat{\Pi}^{(0)}(t)$ and $\widehat{\Pi}_{c}^{(0)}(t)$. 
Let $u(t)$ be a solution of (4.6). We decompose $u(t)$ as

$$
u(t)=\sigma(t) u^{(0)}(t)+u_{1}(t)
$$

where

$$
\begin{aligned}
& \sigma(t)=\left[Q_{0} u(t)\right]=\left\langle u(t), u^{(0) *}\right\rangle, \\
& u_{1}(t)=\widehat{\Pi}_{c}^{(0)}(t) u(t) .
\end{aligned}
$$

Using this decomposition we rewrite problem (4.6). To do so, we define some notation. We write

$$
\widetilde{M}_{\xi^{\prime}}(t)=\widehat{L}_{\xi^{\prime}}(t)-\widehat{L}_{0}(t)=\widetilde{A}_{\xi^{\prime}}+\widetilde{B}_{\xi^{\prime}}(t)
$$

where

$$
\begin{gathered}
\widetilde{A}_{\xi^{\prime}}=\widehat{A}_{\xi^{\prime}}-\widehat{A}_{0} \\
=\left(\begin{array}{ccc}
0 & 0 & 0 \\
0 & \frac{\nu}{\rho_{p}}\left|\xi^{\prime}\right|^{2} I_{n-1}+\frac{\widetilde{\nu}}{\rho_{p}} \xi^{\prime T} \xi^{\prime} & -i \frac{\widetilde{\nu}}{\rho_{p}} \xi^{\prime} \partial_{x_{n}} \\
0 & -i \frac{\widetilde{\nu}}{\rho_{p}} \xi^{\prime} \partial_{x_{n}} & \frac{\nu}{\rho_{p}}\left|\xi^{\prime}\right|^{2}
\end{array}\right), \\
\widetilde{B}_{\xi^{\prime}}(t)=\widehat{B}_{\xi^{\prime}}(t)-\widehat{B}_{0}(t)=\left(\begin{array}{ccc}
i \xi_{1} v_{p}^{1}(t) & i \gamma^{2} \rho_{p}^{T} \xi^{\prime} & 0 \\
i \frac{\widetilde{P}^{\prime}\left(\rho_{p}\right)}{\gamma^{2} \rho_{p}} \xi^{\prime} & i \xi_{1} v_{p}^{1}(t) I_{n-1} & 0 \\
0 & 0 & i \xi_{1} v_{p}^{1}(t)
\end{array}\right) .
\end{gathered}
$$

Substituting $u(t)=\sigma(t) u^{(0)}(t)+u_{1}(t)$ into (4.6), we have

$$
\frac{d}{d t}\left(\sigma(t) u^{(0)}(t)+u_{1}(t)\right)+\widehat{L}_{0}(t)\left(\sigma(t) u^{(0)}(t)+u_{1}(t)\right)+\widetilde{M}_{\xi^{\prime}}(t)\left(\sigma(t) u^{(0)}(t)+u_{1}(t)\right)=0 .
$$

Since $Q_{0} \frac{d}{d t} u^{(0)}(t)=0$, we have $\widehat{\Pi}^{(0)}(t) \frac{d}{d t} u^{(0)}(t)=\left[Q_{0} \frac{d}{d t} u^{(0)}(t)\right] u^{(0)}(t)=0$. Therefore, applying $\widehat{\Pi}^{(0)}(t)$ and $\widehat{\Pi}_{c}^{(0)}(t)$ to this equation, we have

$$
\left\{\begin{aligned}
\left(\frac{d}{d t} \sigma(t)\right) u^{(0)}(t) & +\widehat{\Pi}^{(0)}(t) \widetilde{M}_{\xi^{\prime}}(t)\left(\sigma(t) u^{(0)}(t)+u_{1}(t)\right)=0 \\
\sigma(t)\left(\frac{d}{d t} u^{(0)}(t)+\widehat{L}_{0}(t) u^{(0)}(t)\right) & +\frac{d}{d t} u_{1}(t)+\widehat{L}_{0}(t) u_{1}(t) \\
& +\widehat{\Pi}_{c}^{(0)}(t) \widetilde{M}_{\xi^{\prime}}(t)\left(\sigma(t) u^{(0)}(t)+u_{1}(t)\right)=0 .
\end{aligned}\right.
$$

Since $\widehat{\Pi}^{(0)}(t) \widetilde{M}_{\xi^{\prime}}(t) u=\left[Q_{0} \widetilde{M}_{\xi^{\prime}}(t) u\right] u^{(0)}(t), Q_{0} \widetilde{M}_{\xi^{\prime}}(t)=Q_{0} \widetilde{B}_{\xi^{\prime}}(t)$ and fact that $u^{(0)}(t)$ is solution to (4.13), (4.14) we arrive at

$$
\frac{d}{d t} \sigma(t)+\left[Q_{0} \widetilde{B}_{\xi^{\prime}}(t)\left(\sigma(t) u^{(0)}(t)+u_{1}(t)\right)\right]=0
$$




$$
\frac{d}{d t} u_{1}(t)+\widehat{L}_{0}(t) u_{1}(t)+\widetilde{M}_{\xi^{\prime}}(t)\left(\sigma(t) u^{(0)}(t)+u_{1}(t)\right)-\left[Q_{0} \widetilde{B}_{\xi^{\prime}}(t)\left(\sigma(t) u^{(0)}(t)+u_{1}(t)\right)\right] u^{(0)}(t)=0 .
$$

Proposition 4.2 can be proved by estimating solutions of (4.16)-(4.17). We will employ an energy method to obtain the necessary estimates on solutions of (4.16)-(4.17).

We introduce some notations. For $u={ }^{T}(\phi, w)$ we define $E_{0}(u)$ by

$$
E_{0}(u)=\frac{1}{\gamma^{2}}\left|\sqrt{\frac{\tilde{P}^{\prime}\left(\rho_{p}\right)}{\gamma^{2} \rho_{p}}} \phi\right|_{2}^{2}+\left|\sqrt{\rho_{p}} w\right|_{2}^{2} .
$$

For $v=\phi, v=w={ }^{T}\left(w_{1}, \ldots, w^{n}\right)$ or $v={ }^{T}(\phi, w)$, we define $D_{\xi^{\prime}}(v)$ by

$$
D_{\xi^{\prime}}(v)=\left|\xi^{\prime}\right|^{2}|v|_{2}^{2}+\left|\partial_{x_{n}} v\right|_{2}^{2},
$$

and, for $w={ }^{T}\left(w_{1}, \ldots, w^{n}\right)$, we define $\widetilde{D}_{\xi^{\prime}}(w)$ by

$$
\widetilde{D}_{\xi^{\prime}}(w)=\nu D_{\xi^{\prime}}(w)+\widetilde{\nu}\left|i \xi^{\prime} \cdot w^{\prime}+\partial_{x_{n}} w^{n}\right|_{2}^{2} .
$$

We define $J(t)(u)$ by

$$
J(t)(u)=-2 \operatorname{Re}\left\langle\sigma u^{(0)}(t)+\widehat{\Pi}_{c}^{(0)}(t) u, \widehat{B}_{\xi^{\prime}}(t) \widetilde{Q} \widehat{\Pi}_{c}^{(0)}(t) u\right\rangle,
$$

where $\sigma=\left[Q_{0} u\right]$. We note that there exists a constant $b_{0}>0$ such that

$$
|J(t)(u)| \leq \frac{b_{0} \gamma^{2}}{2 \nu}\left(\frac{\alpha_{0}}{\gamma^{2}}|\sigma|^{2}+E_{0}\left(\widehat{\Pi}_{c}^{(0)}(t) u\right)\right)+\frac{1}{2} \widetilde{D}_{\xi^{\prime}}\left(\widetilde{Q} \widehat{\Pi}_{c}^{(0)}(t) u\right) .
$$

Let $b_{1}$ be a positive constant (to be determined in Proposition 4.10 (ii) below) and define $E_{1}(t)(u)$ by

$$
E_{1}(t)(u)=\left(1+\frac{b_{1} \gamma^{2}}{\nu}\right)\left(\frac{\alpha_{0}}{\gamma^{2}}|\sigma|^{2}+E_{0}\left(\widehat{\Pi}_{c}^{(0)}(t) u\right)\right)+\widetilde{D}_{\xi^{\prime}}\left(\widetilde{Q} \widehat{\Pi}_{c}^{(0)}(t) u\right)+J(t)(u),
$$

where $\sigma=\left[Q_{0} u\right]$.

In what follows we denote the solution $u(t)$ of (4.6) by $u(t)=\sigma(t) u^{(0)}(t)+u_{1}(t)$ with

$$
\sigma(t)=\left[Q_{0} u(t)\right], u_{1}(t)={ }^{T}\left(\phi_{1}(t), w_{1}(t)\right)=\widehat{\Pi}_{c}^{(0)}(t) u
$$

and we often omit " $t$ " of $u(t), \sigma(t)$ and $u_{1}(t)={ }^{T}\left(\phi_{1}(t), w_{1}(t)\right)$ if no confusion occurs.

Proposition 4.9. There exist constants $\gamma_{0}>0$ and $\nu_{0}>0$ such that if $\gamma \geq \gamma_{0}$ and $\nu \geq \nu_{0}$ following estimates hold true:

(i)

$$
\frac{d}{d t}\left(\frac{\alpha_{0}}{\gamma^{2}}|\sigma|^{2}+E_{0}\left(u_{1}\right)\right)+\widetilde{D}_{\xi^{\prime}}\left(w_{1}\right) \leq C\left\{\left(\frac{\nu}{\gamma^{4}}+\frac{1}{\gamma^{2}}\right)\left|\phi_{1}\right|_{2}^{2}+\left(\frac{\nu+\tilde{\nu}}{\gamma^{4}}+\frac{1}{\gamma^{2}}\right)\left|\xi^{\prime}\right|^{2}|\sigma|^{2}\right\},
$$

(ii) there exists constant $b_{1} \geq b_{0}$ such that

$$
\begin{aligned}
& \frac{d}{d t} E_{1}(u)+\left(1+\frac{b_{1} \gamma^{2}}{\nu}\right) \widetilde{D}_{\xi^{\prime}}\left(w_{1}\right)+\left|\sqrt{\rho_{p}} \partial_{t} w_{1}\right|_{2}^{2} \\
& \quad \leq C\left\{\left(\frac{1}{\gamma^{2}}+\frac{1}{\nu}+\frac{\nu^{2}}{\gamma^{4}}\right)\left|\phi_{1}\right|_{2}^{2}+\frac{1}{\gamma^{2}}\left|\xi^{\prime}\right|^{2}\left|\phi_{1}\right|_{2}^{2}+\left(\frac{\nu+\tilde{\nu}}{\gamma^{2}}+\frac{1}{\nu}+\frac{1}{\gamma^{2}}\right)\left|\xi^{\prime}\right|^{2}|\sigma|^{2}+\frac{(\nu+\tilde{\nu})^{2}}{\gamma^{4}}\left|\xi^{\prime}\right|^{4}|\sigma|^{2}\right\},
\end{aligned}
$$


(iii)

$$
\begin{aligned}
\frac{d}{d t} \frac{1}{\gamma^{2}} & \left|\sqrt{\frac{\widetilde{P}^{\prime}\left(\rho_{p}\right)}{\gamma^{2} \rho_{p}}} \partial_{x_{n}} \phi_{1}\right|_{2}^{2}+\frac{1}{\nu+\tilde{\nu}}\left|\frac{\widetilde{P}^{\prime}\left(\rho_{p}\right)}{\gamma^{2}} \partial_{x_{n}} \phi_{1}\right|_{2}^{2} \\
& \leq C\left\{\left(\frac{1}{\nu}\left(\frac{1}{\gamma^{2}}+\frac{1}{(\nu+\tilde{\nu})}\right)+\left|\xi^{\prime}\right|^{2}\right) \widetilde{D}_{\xi^{\prime}}\left(w_{1}\right)+\frac{1}{\nu+\tilde{\nu}}\left|\sqrt{\rho_{p}} \partial_{t} w_{1}\right|_{2}^{2}+\frac{\nu+\tilde{\nu}}{\gamma^{4}}\left|\xi^{\prime}\right|^{2}\left(|\sigma|^{2}+\left|\phi_{1}\right|_{2}^{2}\right)\right\},
\end{aligned}
$$

(iv) if $\frac{\nu+\tilde{\nu}}{\gamma^{2}} \leq \frac{1}{4}$ then

$$
\begin{aligned}
& \frac{d}{d t}|\sigma|^{2}+\frac{\tilde{\alpha}_{0} \gamma^{2}}{\nu} \min \left\{1,\left|\xi^{\prime}\right|^{2}\right\}|\sigma|^{2} \\
& \quad \leq C\left\{\frac{\nu+\tilde{\nu}}{\gamma^{2}}|\sigma|^{2}\left|\xi^{\prime}\right|^{2}+\left(\frac{\nu}{\gamma^{2}}+\frac{\gamma^{2}}{\nu}\right)\left(1+\left|\xi^{\prime}\right|^{2}\right)\left|\phi_{1}\right|_{2}^{2}+\frac{\gamma^{2}}{\nu}\left|\sqrt{\rho_{p}} \partial_{t} w_{1}\right|_{2}^{2}+\frac{\gamma^{2}}{\nu}\left(\frac{1}{\nu}+\widetilde{\nu}\right) \widetilde{D}_{\xi^{\prime}}\left(w_{1}\right)\right\},
\end{aligned}
$$

with some constant $\tilde{\alpha}_{0}>0$.

Proof. Proofs of (i), (iii) and (iv) are same as proofs of [6, Proposition 4.7, 4.9 and 4.10], respectively, so we omit them. We prove (ii) for reader's convenience. In the proof we will often use following lemma.

Lemma 4.10. For each $t$ there hold the following assertions with $C>0$ independent of $t$.

(i) $\left\langle u^{(0)}(t), u_{1}(t)\right\rangle=\left\langle u_{1}^{(0)}(t), u_{1}(t)\right\rangle$,

(ii) $\left|\left[Q_{0} \widetilde{B}_{\xi^{\prime}}(t) u_{0}^{(0)}\right]\right|+\left|\left[Q_{0} \widetilde{B}_{\xi^{\prime}}(t) u_{1}^{(0)}(t)\right]\right| \leq C\left|\xi^{\prime}\right|$

(iii) $\left|\left[Q_{0} \widetilde{B}_{\xi^{\prime}}(t) u_{1}(t)\right]\right| \leq C\left|\xi^{\prime}\right|\left(\left|\phi_{1}(t)\right|_{2}+\gamma^{2}\left|w_{1}^{\prime}(t)\right|_{2}\right)$.

The proof of Lemma 4.10 is straightforward, so we omit it.

Let us proof (ii) from Proposition 4.9. We recall that $u(t)=\sigma(t) u^{(0)}(t)+u_{1}(t)$ satisfies

$$
\frac{d}{d t} u+\widehat{L}_{\xi^{\prime}}(t) u=0
$$

which implies

$$
\left\langle\partial_{t} u, \partial_{t} \widetilde{Q} u_{1}\right\rangle+\left\langle\widehat{L}_{\xi^{\prime}}(t) u, \partial_{t} \widetilde{Q} u_{1}\right\rangle=0 .
$$

Since

$$
\partial_{t} \sigma=-\left[Q_{0} \widetilde{B}_{\xi^{\prime}}\left(\sigma u^{(0)}(t)+u_{1}\right)\right]
$$

and

$$
\left\langle u^{(0)}(t), \partial_{t} \widetilde{Q} u_{1}\right\rangle=\left\langle u_{1}^{(0)}(t), \partial_{t} \widetilde{Q} u_{1}\right\rangle,
$$

by using Lemma 4.10, we have

$$
\begin{aligned}
& \operatorname{Re}\left\langle\partial_{t} u, \partial_{t} \widetilde{Q} u_{1}\right\rangle \\
& =\operatorname{Re}\left\{\left\langle\partial_{t}\left(\sigma u^{(0)}(t)\right), \partial_{t} \widetilde{Q} u_{1}\right\rangle+\left\langle\partial_{t} u_{1}, \partial_{t} \widetilde{Q} u_{1}\right\rangle\right\} \\
& =\operatorname{Re}\left\{-\left[Q_{0} \widetilde{B}_{\xi^{\prime}}\left(\sigma u^{(0)}(t)+u_{1}\right)\right]\left\langle u_{1}^{(0)}(t), \partial_{t} \widetilde{Q} u_{1}\right\rangle+\left|\sqrt{\rho_{p}} \partial_{t} w_{1}\right|_{2}^{2}+\left\langle\sigma \partial_{t} u^{(0)}(t), \partial_{t} \widetilde{Q} u_{1}\right\rangle\right\} \\
& \quad \geq \frac{3}{4}\left|\sqrt{\rho_{p}} \partial_{t} w_{1}\right|_{2}^{2}-C\left\{\frac{\left|\xi^{\prime}\right|^{2}}{\gamma^{4}}\left(|\sigma|^{2}+\left|\phi_{1}\right|_{2}^{2}\right)+\frac{1}{\nu} \widetilde{D}_{\xi^{\prime}}\left(w_{1}\right)\right\}+\operatorname{Re}\left\langle\sigma \partial_{t} u^{(0)}(t), \partial_{t} \widetilde{Q} u_{1}\right\rangle .
\end{aligned}
$$


Since $\widehat{L}_{0}(t) u^{(0)}=-\partial_{t} u^{(0)}$ and $\widehat{B}_{0}(t) u^{(0)}=0$, we see that

$$
\begin{aligned}
\left\langle\widehat{L}_{\xi^{\prime}}(t) u, \partial_{t} \widetilde{Q} u_{1}\right\rangle & \\
= & \left\langle\widetilde{M}_{\xi^{\prime}}(t)\left(\sigma u^{(0)}(t)\right), \partial_{t} \widetilde{Q} u_{1}\right\rangle+\left\langle\widehat{L}_{\xi^{\prime}}(t) u_{1}, \partial_{t} \widetilde{Q} u_{1}\right\rangle+\left\langle\sigma \widehat{L}_{0}(t) u^{(0)}(t), \partial_{t} \widetilde{Q} u_{1}\right\rangle \\
= & \left\langle\widetilde{A}_{\xi^{\prime}}\left(\sigma u^{(0)}(t)\right), \partial_{t} \widetilde{Q} u_{1}\right\rangle+\left\langle\widetilde{B}_{\xi^{\prime}}(t)\left(\sigma u^{(0)}(t)\right), \partial_{t} \widetilde{Q} u_{1}\right\rangle \\
& +\left\langle\widehat{A}_{\xi^{\prime}} u_{1}, \partial_{t} \widetilde{Q} u_{1}\right\rangle+\left\langle\widehat{B}_{\xi^{\prime}}(t) u_{1}, \partial_{t} \widetilde{Q} u_{1}\right\rangle+\left\langle\widehat{C}_{0}(t) u_{1}, \partial_{t} \widetilde{Q} u_{1}\right\rangle+\left\langle\sigma \widehat{L}_{0}(t) u^{(0)}(t), \partial_{t} \widetilde{Q} u_{1}\right\rangle \\
= & \left\langle\widetilde{A}_{\xi^{\prime}}\left(\sigma u^{(0)}(t)\right), \partial_{t} \widetilde{Q} u_{1}\right\rangle+\left\langle\widehat{B}_{\xi^{\prime}}(t)\left(\sigma u^{(0)}(t)+u_{1}\right), \partial_{t} \widetilde{Q} u_{1}\right\rangle \\
& +\left\langle\widehat{A}_{\xi^{\prime}} u_{1}, \partial_{t} \widetilde{Q} u_{1}\right\rangle+\left\langle\widehat{C}_{0}(t) u_{1}, \partial_{t} \widetilde{Q} u_{1}\right\rangle-\left\langle\sigma \partial_{t} u^{(0)}(t), \partial_{t} \widetilde{Q} u_{1}\right\rangle .
\end{aligned}
$$

We will show

$$
\begin{aligned}
\operatorname{Re} & \left\{\left\langle\widehat{B}_{\xi^{\prime}}(t)\left(\sigma u^{(0)}(t)+u_{1}\right), \partial_{t} \widetilde{Q} u_{1}\right\rangle+\left\langle\widehat{A}_{\xi^{\prime}} u_{1}, \partial_{t} \widetilde{Q} u_{1}\right\rangle\right\} \\
\geq & \frac{1}{2} \frac{d}{d t}\left(\widetilde{D}_{\xi^{\prime}}\left(w_{1}\right)+J(t)(u)\right)-\varepsilon\left|\sqrt{\rho_{p}} \partial_{t} w_{1}\right|_{2}^{2} \\
& \quad-C\left\{\frac{\left|\xi^{\prime}\right|^{2}}{\gamma^{2}}\left(|\sigma|^{2}+\left|\phi_{1}\right|_{2}^{2}\right)+\frac{\gamma^{2}}{\nu} \widetilde{D}_{\xi^{\prime}}\left(w_{1}\right)+\frac{1}{\varepsilon \nu} \widetilde{D}_{\xi^{\prime}}\left(w_{1}\right)\right\}
\end{aligned}
$$

for any $\varepsilon>0$.

It is easy to see that

$$
\operatorname{Re}\left\langle\widehat{A}_{\xi^{\prime}} u_{1}, \partial_{t} \widetilde{Q} u_{1}\right\rangle=\frac{1}{2} \frac{d}{d t} \widetilde{D}_{\xi^{\prime}}\left(w_{1}\right) .
$$

Since $\widehat{B}_{\xi^{\prime}}^{*}(t)=-\widehat{B}_{\xi^{\prime}}(t)$, we have

$$
\begin{aligned}
\left\langle\widehat{B}_{\xi^{\prime}}(t)\left(\sigma u^{(0)}(t)\right), \partial_{t} \widetilde{Q} u_{1}\right\rangle= & -\frac{d}{d t}\left\langle\sigma u^{(0)}(t), \widehat{B}_{\xi^{\prime}}(t) \widetilde{Q} u_{1}\right\rangle+\left\langle\partial_{t} \sigma u^{(0)}(t), \widehat{B}_{\xi^{\prime}}(t) \widetilde{Q} u_{1}\right\rangle \\
& +\left\langle\sigma \partial_{t} u_{1}^{(0)}(t), \widehat{B}_{\xi^{\prime}}(t) \widetilde{Q} u_{1}\right\rangle+\left\langle\sigma u^{(0)}(t),\left(\partial_{t} \widehat{B}_{\xi^{\prime}}(t)\right) \widetilde{Q} u_{1}\right\rangle .
\end{aligned}
$$

By (4.16), we have

$$
\partial_{t} \sigma=-\left[Q_{0} \widetilde{B}_{\xi^{\prime}}(t)\left(\sigma u^{(0)}(t)+u_{1}\right)\right] .
$$

Lemma 4.10 then implies

$$
\begin{aligned}
& \left|\left\langle\partial_{t} \sigma u^{(0)}(t), \widehat{B}_{\xi^{\prime}}(t) \widetilde{Q} u_{1}\right\rangle\right| \\
& \quad \leq\left|\left[Q_{0} \widetilde{B}_{\xi^{\prime}}(t)\left(\sigma u^{(0)}(t)+u_{1}\right)\right]\right|\left|\left\langle u^{(0)}(t), \widehat{B}_{\xi^{\prime}}(t) \widetilde{Q} u_{1}\right\rangle\right| \leq C\left\{\frac{\left|\xi^{\prime}\right|^{2}}{\gamma^{2}}\left(|\sigma|^{2}+\left|\phi_{1}\right|_{2}^{2}\right)+\frac{\gamma^{2}}{\nu} \widetilde{D}_{\xi^{\prime}}\left(w_{1}\right)\right\} .
\end{aligned}
$$

In the same way we get

$$
\left|\left\langle\sigma \partial_{t} u_{1}^{(0)}(t), \widehat{B}_{\xi^{\prime}}(t) \widetilde{Q} u_{1}\right\rangle\right| \leq C\left\{\frac{\left|\xi^{\prime}\right|^{2}}{\gamma^{2}}|\sigma|^{2}+\frac{1}{\gamma^{2} \nu} \widetilde{D}_{\xi^{\prime}}\left(w_{1}\right)\right\},
$$

and

$$
\left|\left\langle\sigma u^{(0)}(t),\left(\partial_{t} \widehat{B}_{\xi^{\prime}}(t)\right) \widetilde{Q} u_{1}\right\rangle\right| \leq \underset{21}{C}\left\{\frac{\left|\xi^{\prime}\right|^{2}}{\gamma^{2}}|\sigma|^{2}+\frac{1}{\gamma^{2} \nu} \widetilde{D}_{\xi^{\prime}}\left(w_{1}\right)\right\} .
$$


Similarly,

$$
\begin{aligned}
& \left\langle\widehat{B}_{\xi^{\prime}}(t) u_{1}, \partial_{t} \widetilde{Q} u_{1}\right\rangle \\
& \quad=-\frac{d}{d t}\left\langle u_{1}, \widehat{B}_{\xi^{\prime}}(t) \widetilde{Q} u_{1}\right\rangle+\left\langle\partial_{t} u_{1}, \widehat{B}_{\xi^{\prime}}(t) \widetilde{Q} u_{1}\right\rangle+\left\langle u_{1},\left(\partial_{t} \widehat{B}_{\xi^{\prime}}(t)\right) \widetilde{Q} u_{1}\right\rangle \\
& \quad=-\frac{d}{d t}\left\langle u_{1}, \widehat{B}_{\xi^{\prime}}(t) \widetilde{Q} u_{1}\right\rangle+\left\langle\partial_{t} Q_{0} u_{1}, \widehat{B} \widehat{\xi}^{\prime}(t) \widetilde{Q} u_{1}\right\rangle+\left\langle\partial_{t} \widetilde{Q} u_{1}, \widehat{B}_{\xi^{\prime}}(t) \widetilde{Q} u_{1}\right\rangle+\left\langle u_{1},\left(\partial_{t} \widehat{B_{\xi^{\prime}}}(t)\right) \widetilde{Q} u_{1}\right\rangle .
\end{aligned}
$$

We first estimate the second term on the right of (4.28). By (4.17), we have

$$
\begin{aligned}
\partial_{t} Q_{0} u_{1} & =-Q_{0}\left\{\widehat{L}_{\xi^{\prime}}(t) u_{1}+\widetilde{M}_{\xi^{\prime}}(t)\left(\sigma u^{(0)}(t)\right)-\left[Q_{0} \widetilde{B}_{\xi^{\prime}}(t)\left(\sigma u^{(0)}(t)+u_{1}\right)\right] u^{(0)}\right\} \\
& =-\left\{Q_{0} \widehat{B}_{\xi^{\prime}}(t) u_{1}+Q_{0} \widetilde{B}_{\xi^{\prime}}(t)\left(\sigma u^{(0)}(t)\right)-\left[Q_{0} \widetilde{B}_{\xi^{\prime}}(t)\left(\sigma u^{(0)}(t)+u_{1}\right)\right] u_{0}^{(0)}\right\} .
\end{aligned}
$$

Since $\left\langle\partial_{t} Q_{0} u_{1}, \widehat{B}_{\xi^{\prime}}(t) \widetilde{Q} u_{1}\right\rangle=\left\langle\partial_{t} Q_{0} u_{1}, Q_{0} \widehat{B}_{\xi^{\prime}}(t) \widetilde{Q} u_{1}\right\rangle$, we see from Lemma 4.10 that

$$
\begin{aligned}
\left|\left\langle\partial_{t} Q_{0} u_{1}, \widehat{B}_{\xi^{\prime}}(t) \widetilde{Q} u_{1}\right\rangle\right| & \\
\leq & C\left\{\left|Q_{0} \widehat{B}_{\xi^{\prime}}(t) u_{1}\right|_{2}+\left|Q_{0} \widetilde{B}_{\xi^{\prime}}(t)\left(\sigma u^{(0)}(t)\right)\right|_{2}\right. \\
& \left.+\left|\left[Q_{0} \widetilde{B}_{\xi^{\prime}}(t)\left(\sigma u^{(0)}(t)+u_{1}\right)\right]\right|\left|u_{0}^{(0)}\right|_{2}\right\} \times \frac{1}{\gamma^{2}}\left|Q_{0} \widehat{B}_{\xi^{\prime}}(t) \widetilde{Q} u_{1}\right|_{2} \\
\leq & C\left\{\frac{\left|\xi^{\prime}\right|^{2}}{\gamma^{2}}\left(|\sigma|^{2}+\left|\phi_{1}\right|_{2}^{2}\right)+\frac{\gamma^{2}}{\nu} \widetilde{D}_{\xi^{\prime}}\left(w_{1}\right)\right\} .
\end{aligned}
$$

The third term on the right of (4.28) is estimated as

$$
\left|\left\langle\partial_{t} \widetilde{Q} u_{1}, \widehat{B}_{\xi^{\prime}}(t) \widetilde{Q} u_{1}\right\rangle\right| \leq C\left|\partial_{t} w_{1}\right|_{2}\left|\xi^{\prime}\right|\left|w_{1}\right|_{2} \leq \varepsilon\left|\sqrt{\rho_{p}} \partial_{t} w_{1}\right|_{2}^{2}+\frac{C}{\varepsilon \nu} \widetilde{D}_{\xi^{\prime}}\left(w_{1}\right)
$$

for any $\varepsilon>0$. The fourth term on the right of (4.28) is estimated as

$$
\left|\left\langle u_{1},\left(\partial_{t} \widehat{B}_{\xi^{\prime}}(t)\right) \widetilde{Q} u_{1}\right\rangle\right| \leq C\left|\xi^{\prime}\right|\left|w_{1}\right|_{2}^{2} \leq C \frac{1}{\nu} \widetilde{D}_{\xi^{\prime}}\left(w_{1}\right) .
$$

This, together with (4.29) and (4.30), gives

$$
\begin{aligned}
& \operatorname{Re}\left\langle\widehat{B}_{\xi^{\prime}}(t) u_{1}, \partial_{t} \widetilde{Q} u_{1}\right\rangle \\
& \quad \geq-\frac{d}{d t}\left\langle u_{1}, \widehat{B}_{\xi^{\prime}}(t) \widetilde{Q} u_{1}\right\rangle-\varepsilon\left|\sqrt{\rho_{p}} \partial_{t} w_{1}\right|_{2}^{2}-C\left\{\frac{\left|\xi^{\prime}\right|^{2}}{\gamma^{2}}\left(|\sigma|^{2}+\left|\phi_{1}\right|_{2}^{2}\right)+\left(\frac{\gamma^{2}}{\nu}+\frac{1}{\varepsilon \nu}\right) \widetilde{D}_{\xi^{\prime}}\left(w_{1}\right)\right\},
\end{aligned}
$$

for any $\varepsilon>0$. We thus obtain (4.22) from (4.23)-(4.27) and (4.31).

A straightforward computation gives

$$
\left|\left\langle\widetilde{A}_{\xi^{\prime}}\left(\sigma u^{(0)}(t)\right), \partial_{t} \widetilde{Q} u_{1}\right\rangle\right| \leq \varepsilon\left|\sqrt{\rho_{p}} \partial_{t} w_{1}\right|_{2}^{2}+\frac{C}{\varepsilon} \frac{(\nu+\tilde{\nu})^{2}}{\gamma^{4}}\left|\xi^{\prime}\right|^{2}\left(1+\left|\xi^{\prime}\right|^{2}\right)|\sigma|^{2},
$$

and

$$
\left|\left\langle\widehat{C}_{0}(t) u_{1}, \partial_{t} \widetilde{Q} u_{1}\right\rangle\right| \leq \varepsilon\left|\sqrt{\rho_{p}} \partial_{t} w_{1}\right|_{2}^{2}+\frac{C}{\varepsilon}\left\{\frac{\nu^{2}}{\gamma^{4}}\left|\phi_{1}\right|_{2}^{2}+\frac{1}{\nu} \widetilde{D}_{\xi^{\prime}}\left(w_{1}\right)\right\},
$$


for any $\varepsilon>0$.

Taking $\varepsilon>0$ suitably small, we see from (4.19)-(4.22), (4.32) and (4.33) that

$$
\begin{aligned}
& \frac{1}{2} \frac{d}{d t}\left(\widetilde{D}_{\xi^{\prime}}\left(w_{1}\right)+J(t)(u)\right)+\frac{1}{2}\left|\sqrt{\rho_{p}} \partial_{t} w_{1}\right|_{2}^{2} \\
& \quad \leq C\left\{\frac{\nu^{2}}{\gamma^{4}}\left|\phi_{1}\right|_{2}^{2}+\frac{\left|\xi^{\prime}\right|^{2}}{\gamma^{2}}\left(|\sigma|^{2}+\left|\phi_{1}\right|_{2}^{2}\right)+\frac{(\nu+\tilde{\nu})^{2}}{\gamma^{4}}\left|\xi^{\prime}\right|^{2}\left(1+\left|\xi^{\prime}\right|^{2}\right)|\sigma|^{2}+\left(\frac{\gamma^{2}}{\nu}+\frac{1}{\nu}\right) \widetilde{D}_{\xi^{\prime}}\left(w_{1}\right)\right\} .
\end{aligned}
$$

Adding $2 \times(4.34)$ to $\left(1+\frac{b_{1} \gamma^{2}}{\nu}\right) \times(4.18)$ with suitably large $b_{1} \geq b_{0}$, we obtain the desired estimate. This completes the proof.

Proof of Proposition 4.2. We assume $\left|\xi^{\prime}\right| \leq R_{0}$. We fix $b_{1}$ in $E_{1}(t)(u)$ as in Proposition 4.9 (ii). As in the proof of [6, Proposition 4.2], by making a suitable linear combination of inequalities (i)-(iv) of Proposition 4.9 one can show, that there exist constants $\nu_{0}>0$ and $\gamma_{0}>0$ such that if $\nu \geq \nu_{0}$ and $\gamma^{2} /(\nu+\widetilde{\nu}) \geq \gamma_{0}^{2}$ then

$$
\frac{d}{d t} E_{3}(u)+c_{1} \widetilde{D}_{\xi^{\prime}}\left(w_{1}\right)+c_{2}\left|\sqrt{\rho_{p}} \partial_{t} w_{1}\right|_{2}^{2}+c_{3}\left|\frac{\widetilde{P}^{\prime}\left(\rho_{p}\right)}{\gamma^{2}} \partial_{x_{n}} \phi_{1}\right|_{2}^{2}+c_{4}\left|\xi^{\prime}\right|^{2}|\sigma|_{2}^{2} \leq 0
$$

for $\xi^{\prime}$ with $\left|\xi^{\prime}\right| \leq R_{0}$, where $c_{1}, c_{2}, c_{3}, c_{4}$ are some positive numbers and $E_{3}(u)$ is a quantity equivalent to $E_{0}(u)+D_{\xi^{\prime}}(u)$.

Since $\left|\xi^{\prime}\right| \leq R_{0}$, we see that

$$
\widetilde{D}_{\xi^{\prime}}\left(w_{1}\right)+\left|\frac{\widetilde{P}^{\prime}\left(\rho_{p}\right)}{\gamma^{2}} \partial_{x_{n}} \phi_{1}\right|_{2}^{2}+\left|\xi^{\prime}\right|^{2}|\sigma|_{2}^{2} \geq c_{0}\left|\xi^{\prime}\right|^{2} E_{3}(u)
$$

for some constant $c_{0}>0$. It then follows from (4.35) and (4.36) that

$$
E_{3}(u(t)) \leq e^{-c_{0}\left|\xi^{\prime}\right|^{2}(t-s-4 T)} E_{3}(u(4 T))
$$

for $t-s \geq 4 T,\left|\xi^{\prime}\right| \leq R_{0}$

Let $\widehat{u}_{s}={ }^{T}(\widehat{\phi}, \widehat{w})$ be the solution of problem (4.1)-(4.5). There exist constants $\nu_{0}>0$ and $\gamma_{0}>0$ such that if $\nu \geq \nu_{0}$ and $\gamma^{2} /(\nu+\widetilde{\nu}) \geq \gamma_{0}^{2}$ then we deduce from (4.37) that

$$
\begin{aligned}
& \left|\xi^{\prime}\right|^{2 k}\left|\widehat{u}_{s}\left(\xi^{\prime}, \cdot, t\right)\right|_{H^{1}}^{2} \\
& \quad \leq C\left|\xi^{\prime}\right|^{2 k} e^{-c_{0}\left|\xi^{\prime}\right|^{2(t-s-4 T)} E_{3}\left(\widehat{u}_{s}\left(\xi^{\prime}, \cdot, s+4 T\right)\right),}
\end{aligned}
$$

for $\xi^{\prime}$ with $\left|\xi^{\prime}\right| \leq R_{0}$.

If we integrate (4.38) over $0<r \leq\left|\xi^{\prime}\right|<R_{0}$, then

$$
\left\|\mathscr{V}_{1}(t, s) u_{0}\right\|_{H^{1}} \leq C e^{-d(t-s-4 T)}\left\|\mathscr{U}(s+4 T, s) u_{0}\right\|_{H^{1}},
$$

with $d=\frac{1}{2} c_{0} r^{2}>0$. Applying Theorem 3.1 to estimate $\left\|\mathscr{U}(s+4 T, s) u_{0}\right\|_{H^{1}}$, we have the desired estimate in Proposition 4.2. 


\section{Proof of Theorem 3.2 - 2. Asymptotic behavior}

In this section we prove the asymptotic behaviour as given in (3.2) and (3.3).

Theorem 5.1. There exist $\nu_{0}>0$ and $\gamma_{0}>0$ such that if $\nu \geq \nu_{0}$ and $\gamma^{2} /(\nu+\tilde{\nu}) \geq \gamma_{0}^{2}$ then there exists $r_{0}>0$ such that $\mathscr{V}_{0}(t, s) u_{0}$ can be written as

$$
\mathscr{V}_{0}(t, s) u_{0}=\mathscr{U}^{(0)}(t, s) u_{0}+\mathscr{R}^{(0)}(t, s) u_{0},
$$

where $\mathscr{U}^{(0)}(t, s) u_{0}$ has the properties in Theorem 3.2 (i) and $\mathscr{R}^{(0)}(t, s) u_{0}$ satisfies the estimate (3.4) in Theorem 3.2 (ii) with $\mathscr{U}^{(\infty)}(t, s) u_{0}$ replaced by $\mathscr{R}^{(0)}(t, s) u_{0}$.

Theorem 3.2 immediately follows from Proposition 4.1, Proposition 4.2 and Theorem 5.1 with $r=r_{0}$ and $R=R_{0}$ by setting $\mathscr{U}^{(\infty)}(t, s) u_{0}=\mathscr{R}^{(0)}(t, s) u_{0}+\mathscr{V}_{1}(t, s) u_{0}+\mathscr{V}_{\infty}(t, s) u_{0}$.

To prove Theorem 5.1 we will investigate spectral properties of the operator $\widehat{L}_{\xi^{\prime}}(t)$ in more detail. Since $\widehat{L}_{\xi^{\prime}}(t)$ is periodic in $t$, we will use theory of periodic solutions, namely monodromy operators. Let us introduce some notation. We decompose $\widehat{L}_{\xi^{\prime}}(t)$ as

$$
\widehat{L}_{\xi^{\prime}}(t)=\widehat{L}_{1}+\widehat{M}_{\xi^{\prime}}(t)
$$

where $\xi^{\prime}={ }^{T}\left(\xi_{1}, \cdots, \xi_{n-1}\right)$, and

$$
\widehat{L}_{1}=\widehat{L}_{0}(t)-\widehat{C}_{0}(t)=\left(\begin{array}{ccc}
0 & 0 & \gamma^{2} \partial_{x_{n}}\left(\rho_{p} \cdot\right) \\
0 & -\frac{\nu}{\rho_{p}} \partial_{x_{n}}^{2} I_{n-1} & 0 \\
\partial_{x_{n}}\left(\frac{\widetilde{P}^{\prime}\left(\rho_{p}\right)}{\gamma^{2} \rho_{p}} \cdot\right) & 0 & -\frac{\nu+\tilde{\nu}}{\rho_{p}} \partial_{x_{n}}^{2}
\end{array}\right)
$$

$\widehat{M}_{\xi^{\prime}}(t)=$

$$
\left(\begin{array}{ccc}
i \xi_{1} v_{p}^{1}(t) & i \gamma^{2} \rho_{p} \xi^{\prime} & 0 \\
\frac{\nu}{\gamma^{2} \rho_{p}^{2}}\left(\partial_{x_{n}}^{2} v_{p}^{1}(t)\right) \boldsymbol{e}_{1}^{\prime}+i \frac{\widetilde{P}^{\prime}\left(\rho_{p}\right)}{\gamma^{2} \rho_{p}} \xi^{\prime} & i v_{p}^{1}(t) \xi_{1} I_{n-1}+\frac{\nu}{\rho_{p}}\left|\xi^{\prime}\right|^{2} I_{n-1}+\frac{\widetilde{\nu}}{\rho_{p}} \xi^{\prime T} \xi^{\prime} & \left(\partial_{x_{n}} v_{p}^{1}(t)\right) \boldsymbol{e}_{1}^{\prime}-i \frac{\widetilde{\nu}}{\rho_{p}} \xi^{\prime} \partial_{x_{n}} \\
0 & -i \frac{\widetilde{\nu}}{\rho_{p}} \xi^{\prime} \partial_{x_{n}} & i \xi_{1} v_{p}^{1}(t)+\frac{\nu}{\rho_{p}}\left|\xi^{\prime}\right|^{2}
\end{array}\right) .
$$

We note that

$$
\widehat{M}_{0}(t)=\widehat{C}_{0}(t)=\left(\begin{array}{ccc}
0 & 0 & 0 \\
\frac{\nu}{\gamma^{2} \rho_{p}^{2}}\left(\partial_{x_{n}}^{2} v_{p}^{1}(t)\right) \boldsymbol{e}_{1}^{\prime} & 0 & \left(\partial_{x_{n}} v_{p}^{1}(t)\right) \boldsymbol{e}_{1}^{\prime} \\
0 & 0 & 0
\end{array}\right)
$$

First we investigate properties of solution operator $\widehat{U}_{0}(t, s)$ for

$$
\begin{gathered}
\partial_{t} u+\widehat{L}_{0}(t) u=0, \\
\left.w\right|_{x_{n}=0,1}=\underset{24}{0,\left.u\right|_{t=s}=u_{0},}
\end{gathered}
$$


and, then, by perturbation argument for $\left|\xi^{\prime}\right| \ll 1$, we analyze properties of solution operator $\widehat{U}_{\xi^{\prime}}(t, s)$ for

$$
\begin{gathered}
\partial_{t} u+\widehat{L}_{\xi^{\prime}}(t) u=0, \\
\left.w\right|_{x_{n}=0,1}=0,\left.u\right|_{t=s}=u_{0},
\end{gathered}
$$

where $u={ }^{T}\left(\phi, w^{\prime}, w^{n}\right)$ is an unknown function of $x_{n} \in[0,1]$ and $t-s \geq 0, s \geq 0$, with $w=\left(w^{\prime}, w^{n}\right)$. Let us first focus on the case $\xi^{\prime}=0$. Since (5.1) is also written as

$$
\partial_{t} u+\widehat{L}_{1} u=-\widehat{M}_{0}(t) u
$$

the solution operator $\widehat{U}_{0}(t, s)$ for $(5.1)$ can be written as

$$
\widehat{U}_{0}(t, s) u_{0}=e^{-(t-s) L_{1}} u_{0}-\int_{s}^{t} e^{-(t-z) L_{1}} \widehat{M}_{0}(z) \widehat{U}_{0}(z, s) u_{0} d z .
$$

We state simple, but useful lemma.

Lemma 5.2. Let $u^{(0)}$ be a function defined as

$$
u^{(0)}=\left.u^{(0)}(t)\right|_{t=0} .
$$

Then

$$
u^{(0)}(t)=\widehat{U}_{0}(t, 0) u^{(0)}, \text { for all } t \geq 0 .
$$

Proof. Proof follows easily from Lemma 4.6 and the uniqueness of the solution.

In what follows we will denote $\widehat{U}_{0}(T)=\widehat{U}_{0}(T, 0)$ corresponding monodromy operator.

Definition 5.3. Let us define projections $\widehat{\Pi}^{(0)}$, $\widehat{\Pi}_{c}^{(0)}$ and $\widehat{\Pi}^{(0) *}$ as

$$
\begin{gathered}
\widehat{\Pi}^{(0)} u=\left\langle u, u^{(0) *}\right\rangle u^{(0)}=\left[Q_{0} u\right] u^{(0)}, u \in X, \\
\widehat{\Pi}_{c}^{(0)} u=\left(I-\widehat{\Pi}^{(0)}\right) u, u \in X, \\
\widehat{\Pi}^{(0) *} u=\left\langle u, u^{(0)}\right\rangle u^{(0) *}, u \in X,
\end{gathered}
$$

respectively, with $u^{(0)}$ given in Lemma 5.2 and

$$
u^{(0) *}={ }^{T}\left(\frac{\gamma^{2}}{\alpha_{0}} \phi^{(0)}, 0,0\right) .
$$

Proposition 5.4. Operator $\widehat{\Pi}^{(0)}$ is an eigen-projection for monodromy operator $\widehat{U}_{0}(T)$ for eigenvalue 1 with commuting property

$$
\widehat{U}_{0}(T) \widehat{\Pi}^{(0)} u_{0}=\widehat{\Pi}^{(0)} \widehat{U}_{0}(T) u_{0}=\widehat{\Pi}^{(0)} u_{0}, u_{0} \in X .
$$

Moreover there exists constant $\gamma_{0}>0$ such that if $\gamma \geq \gamma_{0}$ then eigenvalue 1 is simple and spectrum of monodromy operator $\widehat{U}_{0}(T)$ can be decomposed as

$$
\sigma\left(\widehat{U}_{0}(T)\right)=\{1\} \cup\left\{\lambda:|\lambda| \leq \delta_{0}\right\}
$$


where $\delta_{0}$ is a constant satisfying $0<\delta_{0}<1$.

To prove Proposition 5.4 we first obtain decay estimates on $\widehat{U}_{0}(t, 0) \widehat{\Pi}_{c}^{(0)} u_{0}$.

Lemma 5.5. Let $u_{0} \in X$. Then $\widehat{U}_{0}(t, s) \widehat{\Pi}_{c}^{(0)} u_{0}$ which is solution of equation (5.1), is also a solution of

$$
\begin{gathered}
\partial_{t} \widehat{\Pi}_{c}^{(0)} v+\widehat{L}_{1} \widehat{\Pi}_{c}^{(0)} v=-\widehat{M}_{0}(t) \widehat{\Pi}_{c}^{(0)} v \\
\left.\widehat{\Pi}_{c}^{(0)} v\right|_{t=s}=\widehat{\Pi}_{c}^{(0)} u_{0} .
\end{gathered}
$$

Proof. Lemma 5.5 is easily concluded out of following facts:

$$
\begin{gathered}
\widehat{L}_{1}: \widehat{\Pi}_{c}^{(0)} X \rightarrow \widehat{\Pi}_{c}^{(0)} X, \\
\widehat{\Pi}_{c}^{(0)} \widehat{U}_{0}(t, s) \widehat{\Pi}_{c}^{(0)} u_{0}=\widehat{U}_{0}(t, s) \widehat{\Pi}_{c}^{(0)} u_{0}, \\
\widehat{\Pi}_{c}^{(0)} \widehat{U}_{0}(t, s) \widehat{\Pi}^{(0)} u_{0}=0 .
\end{gathered}
$$

In light of Lemma 5.5 we see that to obtain decay estimates on $\widehat{U}_{0}(t, s) \widehat{\Pi}_{c}^{(0)} u_{0}$ it is enough to obtain decay estimates on solution of equation (5.4). To do so, we investigate spectral properties of $\widehat{L}_{1}$ on $\widehat{\Pi}_{c}^{(0)} X$.

Lemma 5.6. There exists a constant $\gamma_{0}>0$ such that if $\gamma \geq \gamma_{0}$ then there exist positive numbers $\eta_{0}$ and $\theta_{0}$ with $\theta_{0} \in\left(\frac{\pi}{2}, \pi\right)$ such that the following estimates hold uniformly for $\lambda \in \rho\left(-\widehat{L}_{1}\right) \cap \Sigma\left(-\eta_{0}, \theta_{0}\right):$

$$
\begin{gathered}
\left|\left(\lambda+\widehat{L}_{1}\right)^{-1} f\right|_{H^{l} \times L^{2}} \leq \frac{C}{1+|\lambda|}|f|_{H^{1} \times L^{2}}, l=0,1, \\
\left|\partial_{x_{n}}^{l} \widetilde{Q}\left(\lambda+\widehat{L}_{1}\right)^{-1} f\right|_{2} \leq \frac{C}{(|\lambda|+1)^{1-\frac{l}{2}}}|f|_{H^{1} \times L^{2}}, \quad l=1,2,
\end{gathered}
$$

for $f \in \widehat{\Pi}_{c}^{(0)} X$.

Proof. Lemma 5.6 is proved in similar way as [6, Lemma 5.2].

We can see that $\widehat{L}_{1}$ depends on $\frac{1}{\gamma^{2}}$ through $\rho_{p}$ (recall $\left.\left|\rho_{p}-1\right| \leq C \frac{1}{\gamma^{2}}\right)$. Let us introduce operator $\widehat{L}_{1,0}$,

$$
\widehat{L}_{1,0}:=\left(\begin{array}{ccc}
0 & 0 & \gamma^{2} \partial_{x_{n}} \\
0 & -\nu \partial_{x_{n}}^{2} I_{n-1} & 0 \\
\partial_{x_{n}} & 0 & -(\nu+\tilde{\nu}) \partial_{x_{n}}^{2}
\end{array}\right)
$$

which naturally arises in case $\gamma=\infty$. We regard $\widehat{L}_{1}$ as a perturbation from $\widehat{L}_{1,0}$ to estimate $\left(\lambda+\widehat{L}_{1}\right)^{-1}$. As for $\widehat{L}_{1,0}$ we have following result. 
Lemma 5.7. There exist positive numbers $\eta_{0}$ and $\theta_{0}$ with $\theta_{0} \in\left(\frac{\pi}{2}, \pi\right)$ such that the following estimates hold uniformly for $\lambda \in \rho\left(-\widehat{L}_{1,0}\right) \cap \Sigma\left(-\eta_{0}, \theta_{0}\right)$ :

$$
\begin{gathered}
\left|\left(\lambda+\widehat{L}_{1,0}\right)^{-1} f\right|_{H^{l} \times L^{2}} \leq \frac{C}{1+|\lambda|}|f|_{H^{l} \times L^{2}}, \quad l=0,1, \\
\left|\partial_{x_{n}}^{l} \widetilde{Q}\left(\lambda+\widehat{L}_{1,0}\right)^{-1} f\right|_{2} \leq \frac{C}{(|\lambda|+1)^{1-\frac{l}{2}}}|f|_{H^{l-1} \times L^{2}}, \quad l=1,2,
\end{gathered}
$$

for $f \in \widehat{\Pi}_{c}^{(0)} X$.

Proof of Lemma 5.7 is similar to the proof of [2, Lemma 3.1 (ii)], so we omit the proof.

We continue the proof of Lemma 5.6. Since $\rho_{p}$ is smooth, strictly positive and $\rho_{p}=$ $1+O\left(\frac{1}{\gamma^{2}}\right)$, we have

$$
\left|\left(\widehat{L}_{1}-\widehat{L}_{1,0}\right) f\right|_{H^{\ell} \times L^{2}} \leq C\left(1+\frac{\nu+\widetilde{\nu}}{\gamma^{2}}\right) \frac{1}{\gamma^{2}}|f|_{H^{1} \times H^{2}}, \quad \ell=0,1
$$

This, together with Lemma 5.7, implies that if $\frac{1}{\gamma^{2}} \ll 1$, then $\Sigma\left(-\frac{\eta_{0}}{2}, \theta_{0}\right) \subset \rho\left(-\widehat{L}_{1}\right)$ and we get the desired estimates.

From Lemma 5.6 we can conclude that for $\gamma \geq \gamma_{0}$ the $-\widehat{L}_{1}$ is sectorial operator on $\widehat{\Pi}_{c}^{(0)} X$ and we can write

$$
e^{-(t-s) \widehat{L}_{1}}=\frac{1}{2 \pi i} \int_{\Gamma} e^{\lambda(t-s)}\left(\lambda+\widehat{L}_{1}\right)^{-1} d \lambda \text { on } \widehat{\Pi}_{c}^{(0)} X
$$

where contour $\Gamma$ is in $\rho\left(-\left.\widehat{L}_{1}\right|_{\widehat{\Pi}_{c}^{(0)} X}\right) \cap \Sigma\left(-\eta_{0}, \theta_{0}\right)$. Using this identity and estimates in Lemma 5.6 we get by standard calculation that

$$
\begin{gathered}
\left|e^{-(t-s) \widehat{L}_{1}} \widehat{\Pi}_{c}^{(0)} u_{0}\right|_{H^{\ell} \times L^{2}} \leq C e^{-d(t-s)}\left|\widehat{\Pi}_{c}^{(0)} u_{0}\right|_{H^{1} \times L^{2}}, \quad \ell=0,1, \\
\left|\partial_{x_{n}} \widetilde{Q} e^{-(t-s) \widehat{L}_{1}} \widehat{\Pi}_{c}^{(0)} u_{0}\right|_{L^{2}} \leq \frac{C}{(t-s)^{\frac{1}{2}}} e^{-d(t-s)}\left|\widehat{\Pi}_{c}^{(0)} u_{0}\right|_{H^{1} \times L^{2}},
\end{gathered}
$$

for some $d$ positive number. Finally we get following decay estimates on $\widehat{U}_{0}(t, s) \widehat{\Pi}_{c}^{(0)} u_{0}$.

Lemma 5.8. There exists a constant $\gamma_{0}>0$ such that if $\gamma \geq \gamma_{0}$ then there exists positive number $d$ such that following decay estimates hold true,

$$
\begin{gathered}
\left|\widehat{U}_{0}(t, s) \widehat{\Pi}_{c}^{(0)} u_{0}\right|_{H^{\ell} \times L^{2}} \leq C e^{-d(t-s)}\left|\widehat{\Pi}_{c}^{(0)} u_{0}\right|_{H^{1} \times L^{2}}, \quad \ell=0,1, \\
\left|\partial_{x_{n}} \widetilde{Q}_{U_{0}}(t, s) \widehat{\Pi}_{c}^{(0)} u_{0}\right|_{L^{2}} \leq C\left((t-s)^{-\frac{1}{2}}+(t-s)^{\frac{1}{2}}\right) e^{-d(t-s)}\left|\widehat{\Pi}_{c}^{(0)} u_{0}\right|_{H^{1} \times L^{2}}
\end{gathered}
$$

Proof. Using identity (5.3)

$$
\widehat{U}_{0}(t, s) \widehat{\Pi}_{c}^{(0)} u_{0}=e^{-(t-s) L_{1}} \widehat{\Pi}_{c}^{(0)} u_{0}-\int_{s}^{t} e^{-(t-z) L_{1}} \widehat{M}_{0}(z) \widehat{U}_{0}(z, s) \widehat{\Pi}_{c}^{(0)} u_{0} d z
$$


we have $\left|\widehat{U}_{0}(t, s) \widehat{\Pi}_{c}^{(0)} u_{0}\right|_{H^{\ell} \times L^{2}} \leq\left|e^{-(t-s) L_{1}} \widehat{\Pi}_{c}^{(0)} u_{0}\right|_{H^{\ell} \times L^{2}}+\left|\int_{s}^{t} e^{-(t-z) L_{1}} \widehat{M}_{0}(z) \widehat{U}_{0}(z, s) \widehat{\Pi}_{c}^{(0)} u_{0} d z\right|_{H^{\ell} \times L^{2}}$.

From the form of $\widehat{M}_{0}(z)$ we see that $\widehat{M}_{0}(z)=Q^{\prime} \widehat{M}_{0}(z)\left(I-Q^{\prime}\right)$ and we can rewrite above right-hand side as

$$
=\left|e^{-(t-s) L_{1}} \widehat{\Pi}_{c}^{(0)} u_{0}\right|_{H^{\ell} \times L^{2}}+\left|\int_{s}^{t} e^{-(t-z) L_{1}} Q^{\prime} \widehat{M}_{0}(z)\left(I-Q^{\prime}\right) \widehat{U}_{0}(z, s) \widehat{\Pi}_{c}^{(0)} u_{0} d z\right|_{H^{\ell} \times L^{2}}
$$

It is easy to observe that

$$
e^{-(t-z) L_{1}} Q^{\prime}=e^{-(t-z) \nu A} Q^{\prime},
$$

and from (5.3) and form of $\widehat{M}_{0}(z)$ we see that

$$
\left(I-Q^{\prime}\right) \widehat{U}_{0}(z, s)=\left(I-Q^{\prime}\right) e^{-(z-s) L_{1}} .
$$

Using these two equations and (5.5) we get

$$
\begin{aligned}
\left|\widehat{U}_{0}(t, s) \widehat{\Pi}_{c}^{(0)} u_{0}\right|_{H^{\ell} \times L^{2}} & \\
& \leq\left|e^{-(t-s) L_{1}} \widehat{\Pi}_{c}^{(0)} u_{0}\right|_{H^{\ell} \times L^{2}}+\left|\int_{s}^{t} e^{-(t-z) \nu A} \widehat{M}_{0}(z)\left(I-Q^{\prime}\right) e^{-(z-s) \widehat{L}_{1}} \widehat{\Pi}_{c}^{(0)} u_{0} d z\right|_{L^{2}} \\
& \leq C e^{-d(t-s)}\left|\widehat{\Pi}_{c}^{(0)} u_{0}\right|_{H^{1} \times L^{2}}+C \int_{s}^{t} e^{-(t-z) \frac{\nu}{2}} \widehat{M}_{0}^{\infty}\left|e^{-(z-s) \widehat{L}_{1}} \widehat{\Pi}_{c}^{(0)} u_{0}\right|_{L^{2} \times L^{2}} d z
\end{aligned}
$$

where $\widehat{M}_{0}^{\infty}:=\sup _{z}\left|\widehat{M}_{0}(z)\right|_{L^{2}} \leq C\left(1+\frac{\nu}{\gamma^{2}}\right)$. Thanks to (5.5), we have

$$
\int_{s}^{t} e^{-(t-z) \frac{\nu}{2}}\left|e^{-(z-s) \widehat{L}_{1}} \widehat{\Pi}_{c}^{(0)} u_{0}\right|_{L^{2} \times L^{2}} d z \leq \int_{s}^{t} e^{-\left[(t-z) \frac{\nu}{2}+(z-s) d\right]} d z\left|\widehat{\Pi}_{c}^{(0)} u_{0}\right|_{H^{1} \times L^{2}} .
$$

Therefore, with $\widetilde{d}=\min \left\{d, \frac{\nu}{2}\right\}$, we finally get

$$
\left|\widehat{U}_{0}(t, s) \widehat{\Pi}_{c}^{(0)} u_{0}\right|_{H^{\ell} \times L^{2}} \leq C e^{-\widetilde{d}(t-s)}\left|\widehat{\Pi}_{c}^{(0)} u_{0}\right|_{H^{1} \times L^{2}} .
$$

As for second inequality, we follow calculations above using (5.6) to get

$$
\begin{aligned}
\left|\partial_{x_{n}} \widetilde{Q} \widehat{U}_{0}(t, s) \widehat{\Pi}_{c}^{(0)} u_{0}\right|_{L^{2}} \\
\quad \leq\left|\partial_{x_{n}} \widetilde{Q} e^{-(t-s) L_{1}} \widehat{\Pi}_{c}^{(0)} u_{0}\right|_{L^{2}}+\left|\int_{s}^{t} \partial_{x_{n}} \widetilde{Q} e^{-(t-z) L_{1}} \widehat{M}_{0}(z) \widehat{U}_{0}(z, s) \widehat{\Pi}_{c}^{(0)} u_{0} d z\right|_{L^{2}} \\
\quad \leq \frac{C}{(t-s)^{\frac{1}{2}}} e^{-d(t-s)}\left|\widehat{\Pi}_{c}^{(0)} u_{0}\right|_{H^{1} \times L^{2}}+C \int_{s}^{t} \frac{1}{(t-z)^{\frac{1}{2}}} e^{-(t-z) \frac{\nu}{2}} \widehat{M}_{0}^{\infty}\left|e^{-(z-s) \widehat{L}_{1}} \widehat{\Pi}_{c}^{(0)} u_{0}\right|_{L^{2}} d z \\
\quad \leq C\left((t-s)^{-\frac{1}{2}}+(t-s)^{\frac{1}{2}}\right) e^{-\widetilde{d}(t-s)}\left|\widehat{\Pi}_{c}^{(0)} u_{0}\right|_{H^{1} \times L^{2}} .
\end{aligned}
$$


Proof of Proposition 5.4. We already know that $\widehat{U}_{0}(t, 0) \widehat{\Pi}^{(0)} u_{0}$ is a periodic solution of (5.1) which is equivalent to the fact that $\widehat{U}_{0}(T) \widehat{\Pi}^{(0)} u_{0}=\widehat{\Pi}^{(0)} u_{0}$ which is equivalent to the fact that 1 is an eigenvalue of monodromy operator $\widehat{U}_{0}(T)$. To prove that 1 is simple we first show that if

$$
\left(\widehat{U}_{0}(T)-1\right) u=0, \quad u \neq 0,
$$

then $u=C u^{(0)}$ for some $C \in \mathbb{C}$. Let us decompose $u$ using projections $\widehat{\Pi}^{(0)}$ and $\widehat{\Pi}_{c}^{(0)}$,

$$
u=C u^{(0)}+u_{1}, \quad \widehat{\Pi}_{c}^{(0)} u_{1}=u_{1} .
$$

Since $(5.8)$ is equivalent to the fact that $\widehat{U}_{0}(t, 0) u$ is a periodic solution of $(5.1), \widehat{U}_{0}(t, 0) u_{1}$ must also be a periodic solution of (5.1). But combining the decay estimate (5.7) and periodicity of $\widehat{U}_{0}(t, 0) u_{1}$ we get $u_{1}=0$.

Furthermore, we apply $\widehat{\Pi}^{(0)}$ to

$$
\left(\widehat{U}_{0}(T)-1\right) u=u^{(0)}
$$

and we get

$$
\left[Q_{0}\left(\widehat{U}_{0}(T)-1\right) u\right]=\left[Q_{0} u^{(0)}\right]=1,
$$

which yields $0=[\phi(T)]-\left[\phi_{0}\right]=1$. We conclude that $u$ satisfying (5.9) cannot exist. So 1 is simple.

Let us compute a spectral radius of $\widehat{U}_{0}(T)$ on complementary space $\widehat{\Pi}_{c}^{(0)} X$. Using the decay estimate (5.7) we have

$$
\begin{gathered}
r\left(\left.\widehat{U}_{0}(T)\right|_{\widehat{\Pi}_{c}^{(0)} X}\right)=r\left(\widehat{\Pi}_{c}^{(0)} \widehat{U}_{0}(T) \widehat{\Pi}_{c}^{(0)}\right)=\lim _{n \rightarrow \infty}\left|\left(\widehat{\Pi}_{c}^{(0)} \widehat{U}_{0}(T, 0) \widehat{\Pi}_{c}^{(0)}\right)^{n}\right|_{L(X)}^{\frac{1}{n}} \\
=\lim _{n \rightarrow \infty}\left|\widehat{\Pi}_{c}^{(0)} \widehat{U}_{0}(n T, 0) \widehat{\Pi}_{c}^{(0)}\right|_{L(X)}^{\frac{1}{n}} \leq \lim _{n \rightarrow \infty} C^{\frac{1}{n}} e^{-d T} \leq e^{-d T}<1 .
\end{gathered}
$$

Thus we proved the decomposition for $\sigma\left(\widehat{U}_{0}(T)\right)$.

So we can see that solution $u$ to $(5.1)$ with $\left.u\right|_{t=s}=u_{0}$ can be decomposed into $\widehat{U}_{0}(t, s) \widehat{\Pi}^{(0)} u_{0}$ and $\widehat{U}_{0}(t, s) \widehat{\Pi}_{c}^{(0)} u_{0}$, where $\widehat{\Pi}^{(0)} u_{0}$ gives periodic solution $\widehat{U}_{0}(t, s) \widehat{\Pi}^{(0)} u_{0}$ to $(5.1)$ and $\widehat{U}_{0}(t, s) \widehat{\Pi}_{c}^{(0)} u_{0}$ decays exponentially.

In what follows we will need estimates on the whole solution of (5.1).

Lemma 5.9. There exists a constant $\gamma_{0}>0$ such that if $\gamma \geq \gamma_{0}$ then there exists positive number $d$ such that following estimates hold true for $\widehat{U}_{0}(t, s) \widehat{u}_{0}$,

$$
\begin{gathered}
\left|\widehat{U}_{0}(t, s) \widehat{u}_{0}\right|_{H^{\ell} \times L^{2}} \leq C e^{-d(t-s)}\left|\widehat{u}_{0}\right|_{H^{1} \times L^{2}}+C\left|\widehat{\phi}_{0}\right|_{L^{2}}, \quad l=0,1, \\
\left|\partial_{x_{n}} \widetilde{Q} \widehat{U}_{0}(t, s) \widehat{u}_{0}\right|_{L^{2}} \leq C e^{-d(t-s)}\left((t-s)^{-\frac{1}{2}}+(t-s)^{\frac{1}{2}}\right)\left|\widehat{u}_{0}\right|_{H^{1} \times L^{2}}+C\left|\widehat{\phi}_{0}\right|_{L^{2}} .
\end{gathered}
$$

Using decomposition

$$
\widehat{U}_{0}(t, s) \widehat{u}_{0}=\left[\widehat{\phi}_{0}\right] u^{(0)}+\widehat{U}_{0}(t, s) \widehat{\Pi}_{c}^{(0)} \widehat{u}_{0}
$$


we can obtain estimates in Lemma 5.9 by straightforward calculation, so we omit the proof.

We are in position to start investigation of $\widehat{U}_{\xi^{\prime}}(t, s)$. Since $(5.2)$ can be written as

$$
\begin{aligned}
\partial_{t} u+\widehat{L}_{1} u & =-\widehat{M}_{\xi^{\prime}}(t) u, \\
\left.u\right|_{t=s} & =u_{0},
\end{aligned}
$$

we can write $\widehat{U}_{\xi^{\prime}}(t, s)$ in a form of perturbation of $e^{-(t-s) L_{1}}$ as

$$
\widehat{U}_{\xi^{\prime}}(t, s) u_{0}=e^{-(t-s) L_{1}} u_{0}-\int_{s}^{t} e^{-(t-z) L_{1}} \widehat{M}_{\xi^{\prime}}(z) \widehat{U}_{\xi^{\prime}}(z, s) u_{0} d z .
$$

By (5.3) we already have

$$
\widehat{U}_{0}(t, s) u_{0}=e^{-(t-s) L_{1}} u_{0}-\int_{s}^{t} e^{-(t-z) L_{1}} \widehat{M}_{0}(z) \widehat{U}_{0}(z, s) u_{0} d z
$$

Let us define difference operator $\widehat{V}_{\xi^{\prime}}$ as

$$
\widehat{V}_{\xi^{\prime}}(t, s)=\widehat{U}_{\xi^{\prime}}(t, s)-\widehat{U}_{0}(t, s)
$$

Then we can write

$$
\widehat{V}_{\xi^{\prime}}(t, s) u_{0}=-\int_{s}^{t} e^{-(t-z) L_{1}}\left\{\left(\widehat{M}_{\xi^{\prime}}(z)-\widehat{M}_{0}(z)\right) \widehat{U}_{\xi^{\prime}}(z, s)+\widehat{M}_{0}(z) \widehat{V}_{\xi^{\prime}}(z, s)\right\} u_{0} d z
$$

It follows that $v=\widehat{V}_{\xi^{\prime}}(t, s) u_{0}$ is a solution of the following problem:

$$
\partial_{t} v+\widehat{L}_{1} v=-\widehat{M}_{0}(t) v-\left(\widehat{M}_{\xi^{\prime}}(t)-\widehat{M}_{0}(t)\right) u,\left.\quad v\right|_{t=s}=0
$$

with $u=\widehat{U}_{\xi^{\prime}}(t, s) u_{0}$, namely,

$$
\partial_{t} v+\widehat{L}_{0}(t) v=-\left(\widehat{M}_{\xi^{\prime}}(t)-\widehat{M}_{0}(t)\right) u,\left.\quad v\right|_{t=s}=0 .
$$

Therefore, in terms of $\widehat{U}_{0}(t, s)$, a solution of (5.10) can be written as

$$
v(t, s)=\widehat{V}_{\xi^{\prime}}(t, s) u_{0}=-\int_{s}^{t} \widehat{U}_{0}(t, z)\left(\widehat{M}_{\xi^{\prime}}(z)-\widehat{M}_{0}(z)\right) \widehat{U}_{\xi^{\prime}}(z, s) u_{0} d z .
$$

Finally we get following equation for $\widehat{V}_{\xi^{\prime}}$,

$$
\begin{aligned}
\widehat{V}_{\xi^{\prime}}(t, s) u_{0}= & -\int_{s}^{t} \widehat{U}_{0}(t, z)\left(\widehat{M}_{\xi^{\prime}}(z)-\widehat{M}_{0}(z)\right) \widehat{U}_{\xi^{\prime}}(z, s) u_{0} d z \\
= & -\int_{s}^{t} \widehat{U}_{0}(t, z)\left(\widehat{M}_{\xi^{\prime}}(z)-\widehat{M}_{0}(z)\right) \widehat{U}_{0}(z, s) u_{0} d z \\
& -\int_{s}^{t} \widehat{U}_{0}(t, z)\left(\widehat{M}_{\xi^{\prime}}(z)-\widehat{M}_{0}(z)\right) \widehat{V}_{\xi^{\prime}}(z, s) u_{0} d z
\end{aligned}
$$


Let us define operator $\widehat{S}_{\xi^{\prime}}$ as

$$
\left(\widehat{S}_{\xi^{\prime}} V\right)(t) u_{0}=-\int_{0}^{t} \widehat{U}_{0}(t, s)\left(\widehat{M}_{\xi^{\prime}}(s)-\widehat{M}_{0}(s)\right) V(s) u_{0} d s,
$$

where $V \in L(X, Y)$ is defined as

$$
V: u_{0} \in X \mapsto V(s) u_{0} \in Y
$$

with

$$
Y=\left\{u={ }^{T}(\phi, w) \in C([0, T]: X): \partial_{x_{n}} w \in C\left((0, T]: L^{2}(0,1)\right),|u|_{Y}<\infty\right\}
$$

and

$$
|u|_{Y}=\sup _{z \in[0, T]}\left(|u(z)|_{H^{1} \times L^{2}}+z^{\frac{1}{2}}\left|\partial_{x_{n}} w(z)\right|_{L^{2}}\right)
$$

We see that

$$
\widehat{S}_{\xi^{\prime}}: L(X, Y) \rightarrow L(X, Y) .
$$

We note that norm on $L(X, Y)$ is defined as

$$
|V|_{L(X, Y)}=\sup _{u_{0} \in X,\left|u_{0}\right|_{X} \leq 1}\left|V(\cdot) u_{0}\right|_{Y}
$$

Denoting $\widehat{V}_{\xi^{\prime}}(\cdot, 0)$ and $\widehat{U}_{\xi^{\prime}}(\cdot, 0)$ as $\widehat{V}_{\xi^{\prime}}$ and $\widehat{U}_{\xi^{\prime}}$, respectively, we immediately see that

$$
\widehat{V}_{\xi^{\prime}}, \widehat{U}_{\xi^{\prime}} \in L(X, Y)
$$

We rewrite (5.12) in operator form as

$$
\left(I-\widehat{S}_{\xi^{\prime}}\right) \widehat{V}_{\xi^{\prime}}=\widehat{S}_{\xi^{\prime}} \widehat{U}_{0} .
$$

Now we see that if we have $\left|\widehat{S}_{\xi^{\prime}}\right|_{L(L(X, Y))}<1$ for Banach space $L(X, Y)$ we get equation

$$
\widehat{V}_{\xi^{\prime}}=\left(I-\widehat{S}_{\xi^{\prime}}\right)^{-1} \widehat{S}_{\xi^{\prime}} \widehat{U}_{0}=\sum_{n=1}^{\infty} \widehat{S}_{\xi^{\prime}}^{n} \widehat{U}_{0}
$$

which immediately implies formula for $\widehat{U}_{\xi^{\prime}}$,

$$
\widehat{U}_{\xi^{\prime}}=\widehat{U}_{0}+\sum_{n=1}^{\infty} \widehat{S}_{\xi^{\prime}}^{n} \widehat{U}_{0}
$$

Proposition 5.10. There exists a constant $\gamma_{0}>0$ such that if $\gamma \geq \gamma_{0}$ then there exists $r_{0}>0\left(r_{0}=\frac{C}{\gamma^{2}+\nu+\widetilde{\nu}}\right)$ such that if $\left|\xi^{\prime}\right|<r_{0}$ then $\left|\widehat{S}_{\xi^{\prime}}\right| L(L(X, Y))<1$ and therefore $\left(I-\widehat{S}_{\xi^{\prime}}\right)^{-1}$ exists and associated Neumann series converges, so we can write

$$
\widehat{U}_{\xi^{\prime}}=\widehat{U}_{0}+\sum_{n=1}^{\infty} \widehat{S}_{\xi^{\prime}}^{n} \widehat{U}_{0}
$$


Proof. Let $u_{0} \in X$. We expand $\widehat{S}_{\xi^{\prime}}$ as

$$
\widehat{S}_{\xi^{\prime}}=\sum_{j=1}^{n-1} \xi_{j} \widehat{S}_{1}^{(1)}+\sum_{j, k=1}^{n-1} \xi_{j} \xi_{k} \widehat{S}_{j k}^{(2)},
$$

with

$$
\begin{aligned}
& \left(\widehat{S}_{j}^{(1)} V\right)(t) u_{0}=-\int_{0}^{t} \widehat{U}_{0}(t, s) \widehat{M}_{j}^{(1)}(s) V(s) u_{0} d s \\
& \left(\widehat{S}_{j k}^{(2)} V\right)(t) u_{0}=-\int_{0}^{t} \widehat{U}_{0}(t, s) \widehat{M}_{j k}^{(2)}(s) V(s) u_{0} d s
\end{aligned}
$$

where

$$
\begin{gathered}
\widehat{M}_{1}^{(1)}(s)=\left(\begin{array}{ccc}
i v_{p}^{1}(s) & i \gamma^{2} \rho_{p} \boldsymbol{e}_{1}^{\prime} & 0 \\
i \frac{\widetilde{P}^{\prime}\left(\rho_{p}\right)}{\gamma^{2} \rho_{p}} \boldsymbol{e}_{1}^{\prime} & i v_{p}^{1}(s) I_{n-1} & -i \frac{\tilde{\nu}}{\rho_{p}} \partial_{x_{n}} \boldsymbol{e}_{1}^{\prime} \\
0 & -i \frac{\tilde{\nu}}{\rho_{p}} \partial_{x_{n}}{ }^{T} \boldsymbol{e}_{1}^{\prime} & i v_{p}^{1}(s)
\end{array}\right), \\
\widehat{M}_{j}^{(1)}(s)=\left(\begin{array}{ccc}
0 & i \gamma^{2} \rho_{p} \boldsymbol{e}_{j}^{\prime} & 0 \\
i \frac{\tilde{P}^{\prime}\left(\rho_{p}\right)}{\gamma^{2} \rho_{p}} \boldsymbol{e}_{j}^{\prime} & 0 & -i \frac{\tilde{\nu}}{\rho_{p}} \partial_{x_{n}} \boldsymbol{e}_{j}^{\prime} \\
0 & -i \frac{\tilde{\nu}}{\rho_{p}} \partial_{x_{n}}{ }^{T} \boldsymbol{e}_{j}^{\prime} & 0
\end{array}\right), \text { for } j=2, \ldots, n-1, \\
\widehat{M}_{j k}^{(2)}(s)=\left(\begin{array}{ccc}
0 & 0 & 0 \\
0 & \frac{\nu}{\rho_{p}} \delta_{j k} I_{n-1}+\frac{\widetilde{\nu}}{\rho_{p}}{ }^{T} \boldsymbol{e}_{j}^{\prime} \boldsymbol{e}_{k}^{\prime} & 0 \\
0 & 0 & \frac{\nu}{\rho_{p}} \delta_{j k}
\end{array}\right), \text { for } j, k=1, \ldots, n-1,
\end{gathered}
$$

and $\boldsymbol{e}_{j}^{\prime}=(0, \ldots, 0, \underbrace{1}_{j-t h}, 0, \ldots, 0), j=1, \ldots, n-1$.

We compute separately estimates on $\left(\widehat{S}_{j}^{(1)} V\right)(t) u_{0}$ and $\left(\widehat{S}_{j k}^{(2)} V\right)(t) u_{0}$. We denote components of $V(\cdot) u_{0}$ as $V(\cdot) u_{0}={ }^{T}(\phi, w)$. For $j=2, \ldots, n-1$ we have

$$
\left|\widehat{S}_{j}^{(1)} V(t) u_{0}\right|_{H^{1} \times L^{2}} \leq \int_{0}^{t}\left|\widehat{U}_{0}(t, s)\left(\begin{array}{c}
i \gamma^{2} \rho_{p} w^{j} \\
i\left(\frac{\widetilde{P}^{\prime}\left(\rho_{p}\right)}{\gamma^{2} \rho_{p}} \phi-\frac{\widetilde{\nu}}{\rho_{p}} \partial_{x_{n}} w^{n}\right) \boldsymbol{e}_{j}^{\prime} \\
-i \frac{\widetilde{\nu}}{\rho_{p}} \partial_{x_{n}} w^{j}
\end{array}\right)\right|_{H^{1} \times L^{2}} d s
$$

Using estimates from Lemma 5.9 we get

$$
\begin{gathered}
\left|\widehat{S}_{j}^{(1)} V(t) u_{0}\right|_{H^{1} \times L^{2}} \leq \int_{0}^{t} C e^{-d(t-s)}\left[\gamma^{2}\left|w^{j}\right|_{H^{1}}+|\phi|_{L^{2}}+\widetilde{\nu}\left(\left|\partial_{x_{n}} w^{n}\right|_{L^{2}}+\left|\partial_{x_{n}} w^{j}\right|_{L^{2}}\right)\right]+C \gamma^{2}\left|w^{j}\right|_{L^{2}} d s \\
\leq \int_{0}^{t} C e^{-d(t-s)}\left[\gamma^{2}\left(1+s^{-\frac{1}{2}}\right)+1+\widetilde{\nu} s^{-\frac{1}{2}}\right]\left|V(\cdot) u_{0}\right|_{Y}+C \gamma^{2}\left|V(\cdot) u_{0}\right|_{Y} d s
\end{gathered}
$$




$$
\leq C\left|V(\cdot) u_{0}\right|_{Y}\left(\gamma^{2}+\widetilde{\nu}\right)(1+t)
$$

By similar computation we obtain estimates on derivative

$$
\begin{gathered}
t^{\frac{1}{2}}\left|\partial_{x_{n}} \widetilde{Q} \widehat{S}_{j}^{(1)} V(t) u_{0}\right|_{L^{2}} \leq t^{\frac{1}{2}} \int_{0}^{t}\left|\partial_{x_{n}} \widetilde{Q} \widehat{U}_{0}(t, s)\left(\begin{array}{c}
i \gamma^{2} \rho_{p} w^{j} \\
i\left(\frac{\widetilde{P}^{\prime}\left(\rho_{p}\right)}{\gamma^{2} \rho_{p}} \phi-\frac{\widetilde{\nu}}{\rho_{p}} \partial_{x_{n}} w^{n}\right) \boldsymbol{e}_{j}^{\prime} \\
-i \frac{\widetilde{\nu}}{\rho_{p}} \partial_{x_{n}} w^{j}
\end{array}\right)\right|_{L^{2}} d s \\
\leq C\left|V(\cdot) u_{0}\right|_{Y}\left(\gamma^{2}+\widetilde{\nu}\right)\left(1+t^{2}\right) .
\end{gathered}
$$

In case $j=1$ we get

$$
\begin{gathered}
\left|\widehat{S}_{1}^{(1)} V(t) u_{0}\right|_{H^{1} \times L^{2}} \leq \int_{0}^{t}\left|\widehat{U}_{0}(t, s)\left(\begin{array}{c}
i v_{p}^{1} \phi+i \gamma^{2} \rho_{p} w^{1} \\
i\left(\frac{\widetilde{P}^{\prime}\left(\rho_{p}\right)}{\gamma^{2} \rho_{p}} \phi-\frac{\widetilde{\nu}}{\rho_{p}} \partial_{x_{n}} w^{n}\right) \boldsymbol{e}_{1}^{\prime}+i v_{p}^{1} w^{\prime} \\
-i \frac{\widetilde{\nu}}{\rho_{p}} \partial_{x_{n}} w^{1}+i v_{p}^{1} w^{n}
\end{array}\right)\right|_{H^{1} \times L^{2}} d s \\
\leq C\left|V(\cdot) u_{0}\right|_{Y}\left(\gamma^{2}+\widetilde{\nu}\right)(1+t),
\end{gathered}
$$

and for derivative we have

$$
t^{\frac{1}{2}}\left|\partial_{x_{n}} \widetilde{Q} \widehat{S}_{1}^{(1)} V(t) u_{0}\right|_{L^{2}} \leq C\left|V(\cdot) u_{0}\right|_{Y}\left(\gamma^{2}+\widetilde{\nu}\right)\left(1+t^{2}\right) .
$$

For $j, k=1, \ldots, n-1$ we have

$$
\left|\widehat{S}_{j k}^{(2)} V(t) u_{0}\right|_{H^{1} \times L^{2}} \leq \int_{0}^{t}\left|\widehat{U}_{0}(t, s)\left(\begin{array}{c}
0 \\
\frac{\nu}{\rho_{p}} \delta_{j k} w^{\prime}+{\frac{\widetilde{\nu}}{\rho_{p}}}^{T} \boldsymbol{e}_{j}^{\prime} w^{k} \\
\frac{\nu}{\rho_{p}} \delta_{j k} w^{n}
\end{array}\right)\right|_{H^{1} \times L^{2}} d s \leq C\left|V(\cdot) u_{0}\right|_{Y}\left(\nu \delta_{j k}+\widetilde{\nu}\right),
$$

and for derivative we obtain

$$
t^{\frac{1}{2}}\left|\partial_{x_{n}} \widetilde{Q} \widehat{S}_{j k}^{(2)} V(t) u_{0}\right|_{H^{1} \times L^{2}} \leq C\left|V(\cdot) u_{0}\right|_{Y}\left(\nu \delta_{j k}+\widetilde{\nu}\right) t(1+t) .
$$

Now we put all estimates together to get

$$
\begin{aligned}
\left|\widehat{S}_{\xi^{\prime}} V(\cdot) u_{0}\right|_{Y} & \leq \sum_{j=1}^{n-1}\left|\xi_{j}\right|\left|\widehat{S}_{1}^{(1)} V(\cdot) u_{0}\right|_{Y}+\sum_{j, k=1}^{n-1}\left|\xi_{j} \xi_{k}\right|\left|\widehat{S}_{j k}^{(2)} V(\cdot) u_{0}\right|_{Y} \\
\leq & C(T)\left|V(\cdot) u_{0}\right|_{Y}\left(\sum_{j=1}^{n-1}\left|\xi_{j}\right|+\sum_{j, k=1}^{n-1}\left|\xi_{j} \xi_{k}\right|\right) .
\end{aligned}
$$

Thus we conclude that there exist $r_{0}>0$ and $0<q<1$ such that if $\left|\xi^{\prime}\right| \leq r_{0}$ then $\left|\widehat{S}_{\xi^{\prime}} V(\cdot) u_{0}\right|_{Y}<q\left|V(\cdot) u_{0}\right|_{Y}$. Therefore, we have

$$
\left|\widehat{S}_{\xi^{\prime}} V\right|_{L(X, Y)}=\sup _{u_{0} \in X,\left|u_{0}\right|_{X} \leq 1}\left|\widehat{S}_{\xi^{\prime}} V(\cdot) u_{0}\right|_{Y} \leq \sup _{u_{0} \in X,\left|u_{0}\right|_{X} \leq 1} q\left|V(\cdot) u_{0}\right|_{Y}=q|V|_{L(X, Y)}
$$


which concludes the proof.

Assuming that $\gamma \geq \gamma_{0}$ and $\left|\xi^{\prime}\right|<r_{0}$ from Proposition 5.10 we can use the expansion (5.13) and rewrite $\widehat{U}_{\xi^{\prime}}(T, 0)=\widehat{U}_{\xi^{\prime}}(T)$ more precisely as

$$
\widehat{U}_{\xi^{\prime}}(T)=\widehat{U}_{0}(T)+\sum_{j=1}^{n-1} \xi_{j} \widehat{S}_{j}^{(1)} \widehat{U}_{0}(T)+\sum_{j, k=1}^{n-1} \xi_{j} \xi_{k}\left(\widehat{S}_{j k}^{(2)}+\widehat{S}_{j}^{(1)} \widehat{S}_{k}^{(1)}\right) \widehat{U}_{0}(T)+o\left(\left|\xi^{\prime}\right|^{2}\right)
$$

Theorem 5.11. There exist positive numbers $\nu_{0}$ and $\gamma_{0}$ such that if $\nu \geq \nu_{0}$ and $\gamma^{2} /(\nu+\tilde{\nu}) \geq$ $\gamma_{0}^{2}$ then there exists $r_{0}>0$ such that for each $\xi^{\prime}$ with $\left|\xi^{\prime}\right| \leq r_{0}$ it holds that

$$
\sigma\left(\widehat{U}_{\xi^{\prime}}(T)\right) \cap\left\{\lambda ;|\lambda-1|<\frac{\eta_{0}}{2}\right\}=\left\{\widehat{\mu}\left(\xi^{\prime}\right)\right\}
$$

where $\widehat{\mu}\left(\xi^{\prime}\right)$ is a simple eigenvalue of $\widehat{U}_{\xi^{\prime}}(T)$ that has the form

$$
\widehat{\mu}\left(\xi^{\prime}\right)=1-i \kappa_{0} \xi_{1} T-\kappa_{1}\left|\xi^{\prime}\right|^{2} T+O\left(\left|\xi^{\prime}\right|^{3}\right) T
$$

as $\left|\xi^{\prime}\right| \rightarrow 0$. Here $\kappa_{0} \in \mathbb{R}, \kappa_{1}>0$ are some constants having the properties given in Theorem $3.2(\mathrm{i})$.

Proof. Theorem 5.11 is proved by applying the analytic perturbation theory from Kato [7]. We here derive the asymptotic of $\widehat{\mu}\left(\xi^{\prime}\right)$ only.

We proceed as in [3, Theorem 5.3]. Since $\widehat{\mu}^{(0)}=1$ is simple, we can see that $\widehat{\mu}\left(\xi^{\prime}\right)$ is simple and $\widehat{\mu}\left(\xi^{\prime}\right)$ is expanded as

$$
\widehat{\mu}_{0}\left(\xi^{\prime}\right)=\widehat{\mu}^{(0)}+\sum_{j=1}^{n-1} \xi_{j} \widehat{\mu}_{j}^{(1)}+\sum_{j, k=1}^{n-1} \xi_{j} \xi_{k} \widehat{\mu}_{j k}^{(2)}+O\left(\left|\xi^{\prime}\right|^{3}\right)
$$

with

$$
\begin{gathered}
\widehat{\mu}_{j}^{(1)}=\left\langle\widehat{T}_{j}^{(1)} u^{(0)}, u^{(0) *}\right\rangle, \\
\widehat{\mu}_{j k}^{(2)}=\left\langle\left(\widehat{T}_{j k}^{(2)}+\widehat{T}_{j k}^{(3)}\right) u^{(0)}, u^{(0) *}\right\rangle-\left\langle\widehat{T}_{j}^{(1)} \widehat{S} \widehat{T}_{k}^{(1)} u^{(0)}, u^{(0) *}\right\rangle,
\end{gathered}
$$

where

$$
\begin{gathered}
\widehat{T}_{j}^{(1)}=\widehat{S}_{j}^{(1)}\left(\widehat{U}_{0}\right)(T)=-\int_{0}^{T} \widehat{U}_{0}(T, s) \widehat{M}_{j}^{(1)}(s) \widehat{U}_{0}(s, 0) d s \\
\widehat{T}_{j k}^{(2)}=\widehat{S}_{j k}^{(2)}\left(\widehat{U}_{0}\right)(T)=-\int_{0}^{T} \widehat{U}_{0}(T, s) \widehat{M}_{j k}^{(2)}(s) \widehat{U}_{0}(s, 0) d s \\
\widehat{T}_{j k}^{(3)}=\widehat{S}_{j}^{(1)}\left(\widehat{S}_{k}^{(1)}\left(\widehat{U}_{0}\right)\right)(T)=\int_{0}^{T} \int_{0}^{s} \widehat{U}_{0}(T, s) \widehat{M}_{j}^{(1)}(s) \widehat{U}_{0}\left(s, s_{1}\right) \widehat{M}_{k}^{(1)}\left(s_{1}\right) \widehat{U}_{0}\left(s_{1}, 0\right) d s_{1} d s, \\
\widehat{S}=\widehat{\Pi}_{c}^{(0)}\left(\left.\left(\widehat{U}_{0}-I\right)\right|_{\widehat{\Pi}_{c}^{(0)} X}\right)^{-1} \widehat{\Pi}_{c}^{(0)} .
\end{gathered}
$$




\section{Proposition 5.12.}

$$
\widehat{\mu}_{j}^{(1)}= \begin{cases}0 & \text { for } j \neq 1, \\ -i \int_{0}^{T} \int_{0}^{1} \phi^{(0)}\left(x_{n}\right) v_{p}^{1}\left(x_{n}, s\right)+\rho_{p}\left(x_{n}\right) w^{(0), 1}\left(x_{n}, s\right) d x_{n} d s & \text { for } j=1 .\end{cases}
$$

Proof of Proposition 5.12. Since

$$
\widehat{U}_{0}(t, 0) u^{(0)}=\left(\begin{array}{c}
\phi^{(0)} \\
-\frac{1}{\gamma^{2}} \int_{-\infty}^{t} e^{-(t-s) \nu A} \frac{\gamma^{2} \alpha_{0}}{\widetilde{P}^{\prime}\left(\rho_{p}\right) \rho_{p}}\left(\partial_{x_{n}}^{2} v_{p}^{1}(s)\right) d s \boldsymbol{e}_{1}^{\prime} \\
0
\end{array}\right)=\left(\begin{array}{c}
\phi^{(0)} \\
\frac{1}{\gamma^{2}} w^{(0), 1}\left(x_{n}, t\right) \boldsymbol{e}_{1}^{\prime} \\
0
\end{array}\right)
$$

and

$$
\widehat{T}_{j}^{(1)} u^{(0)}=-i \int_{0}^{T} \widehat{U}_{0}(T, s)\left(\begin{array}{c}
\left(\phi^{(0)} v_{p}^{1}(s)+\rho_{p} w^{(0), 1}(s)\right) \delta_{1 j} \\
\alpha_{0} \boldsymbol{e}_{j}^{\prime}+v_{p}^{1}(s) \frac{1}{\gamma^{2}} w^{(0), 1}(s) \boldsymbol{e}_{1}^{\prime} \delta_{1 j} \\
-\frac{\widetilde{\nu}}{\gamma^{2} \rho_{p}} \partial_{x_{n}} w^{(0), 1}(s) \delta_{1 j}
\end{array}\right) d s
$$

we can immediately see the relation for $\widehat{\mu}_{j}^{(1)}$.

Let us next compute $\widehat{\mu}_{j k}^{(2)}$. One can easily see that

$$
\begin{gathered}
\left\langle\widehat{T}_{j k}^{(2)} u^{(0)}, u^{(0) *}\right\rangle=0 \text { for all } j, k=1, \ldots, n-1, \\
\left\langle\widehat{T}_{j k}^{(3)} u^{(0)}, u^{(0) *}\right\rangle=0 \text { for } j \neq k, \\
\left\langle\widehat{T}_{j}^{(1)} \widehat{S} \widehat{T}_{k}^{(1)} u^{(0)}, u^{(0) *}\right\rangle=0 \text { for } j \neq k .
\end{gathered}
$$

We thus obtain the following proposition.

Proposition 5.13. For $j \neq k$,

$$
\widehat{\mu}_{j k}^{(2)}=0 .
$$

We next consider $\widehat{\mu}_{j j}^{(2)}$ for $j=2, \ldots, n-1$.

Proposition 5.14. For $j=2, \ldots, n-1, \widehat{\mu}_{j j}^{(2)}$ satisfies

$$
\widehat{\mu}_{j j}^{(2)}=-\frac{\gamma^{2}}{\nu} T\left(K+O\left(\frac{1}{\nu}\right)\right)
$$

with some positive constant $K$.

Proof of Proposition 5.14. For $j=2, \ldots, n-1$ we get

$$
\left\langle\widehat{T}_{j j}^{(3)} u^{(0)}, u^{(0) *}\right\rangle=\alpha_{0}\left\langle\int_{0}^{T} \int_{0}^{s} \widehat{U}_{0}(T, s)\left(\begin{array}{c}
-\gamma^{2} \rho_{p} e^{-\left(s-s_{1}\right) \nu A} \cdot 1 \\
0 \\
\frac{\widetilde{\nu}}{\rho_{p}} \partial_{x_{n}} e^{-\left(s-s_{1}\right) \nu A} \cdot 1
\end{array}\right) d s_{1} d s, u^{(0) *}\right\rangle .
$$


Let us estimate $\left\langle\widehat{T}_{j j}^{(3)} u^{(0)}, u^{(0) *}\right\rangle$ for $j=2, \ldots, n-1$. We first recall that $\left[Q_{0} \widehat{U}_{0}(t, s) u_{0}\right]=\left[\phi_{0}\right]$. Using this fact we can see from (5.15)

$$
\left\langle\widehat{T}_{j j}^{(3)} u^{(0)}, u^{(0) *}\right\rangle=-\gamma^{2} \int_{0}^{T} \int_{0}^{s} \int_{0}^{1} \alpha_{0} \rho_{p} e^{-\left(s-s_{1}\right) \nu A} \cdot 1 d x_{n} d s_{1} d s .
$$

First we estimate from above,

$$
\int_{0}^{T} \int_{0}^{s} \int_{0}^{1} \rho_{p} e^{-\left(s-s_{1}\right) \nu A} \cdot 1 d x_{n} d s_{1} d s \leq \int_{0}^{T} \int_{0}^{s}\left|\rho_{p}\right|_{\infty} C e^{-\left(s-s_{1}\right) \frac{\nu}{2}} d s_{1} d s \leq \frac{1}{\nu} T C .
$$

Second we estimate from below,

$$
\begin{gathered}
\int_{0}^{T} \int_{0}^{s} \int_{0}^{1} \rho_{p} e^{-\left(s-s_{1}\right) \nu A} \cdot 1 d x_{n} d s_{1} d s \stackrel{\nu s=s}{=} \frac{1}{\nu} \int_{0}^{\nu T} \int_{0}^{\frac{s}{\nu}} \int_{0}^{1} \rho_{p} e^{-\left(s-\nu s_{1}\right) A} \cdot 1 d x_{n} d s_{1} d s \\
\stackrel{\nu s_{1}=s_{1}}{=} \frac{1}{\nu^{2}} \int_{0}^{\nu T} \int_{0}^{s} \int_{0}^{1} \rho_{p} e^{-\left(s-s_{1}\right) A} \cdot 1 d x_{n} d s_{1} d s \stackrel{\text { Fubini }}{=} \frac{1}{\nu^{2}} \int_{0}^{\nu T} \int_{s_{1}}^{\nu T} \int_{0}^{1} \rho_{p} e^{-\left(s-s_{1}\right) A} \cdot 1 d x_{n} d s d s_{1} \\
\stackrel{s-s_{1}=\widetilde{s}}{=} \frac{1}{\nu^{2}} \int_{0}^{\nu T} \int_{0}^{\nu T-s_{1}} \int_{0}^{1} \rho_{p} e^{-\widetilde{s} A} \cdot 1 d x_{n} d \widetilde{s} d s_{1} .
\end{gathered}
$$

By the maximum principle, we have $e^{-\widetilde{s} A} \cdot 1 \geq 0$. It then follows

$$
\frac{1}{\nu^{2}} \int_{0}^{\nu T} \int_{0}^{\nu T-s_{1}} \int_{0}^{1} \underbrace{\rho_{p} e^{-\widetilde{s} A} \cdot 1}_{\geq 0} d x_{n} d \widetilde{s} d s_{1} \geq \frac{1}{\nu^{2}} \int_{0}^{\frac{\nu T}{2}} \int_{0}^{\frac{\nu T}{2}} \int_{0}^{1} \rho_{p} e^{-\widetilde{s} A} \cdot 1 d x_{n} d \widetilde{s} d s_{1} .
$$

Supposing $\nu T>2$, we obtain

$$
\begin{gathered}
\frac{1}{\nu^{2}} \int_{0}^{\frac{\nu T}{2}} \int_{0}^{\frac{\nu T}{2}} \int_{0}^{1} \rho_{p} e^{-\widetilde{s} A} \cdot 1 d x_{n} d \widetilde{s} d s_{1} \geq \frac{1}{\nu^{2}} \int_{0}^{\frac{\nu T}{2}} \int_{0}^{1} \int_{0}^{1} \rho_{p} e^{-\widetilde{s} A} \cdot 1 d x_{n} d \widetilde{s} d s_{1} \\
=\frac{T}{2 \nu} \int_{0}^{1} \int_{0}^{1} \rho_{p} e^{-\widetilde{s} A} \cdot 1 d x_{n} d \widetilde{s}
\end{gathered}
$$

Since $\int_{0}^{1} \int_{0}^{1} \rho_{p} e^{-\widetilde{s} A} \cdot 1 d x_{n} d \widetilde{s}>0$, we conclude that

$$
-\frac{\gamma^{2}}{\nu} T C_{1} \leq\left\langle\widehat{T}_{j j}^{(3)} u^{(0)}, u^{(0) *}\right\rangle \leq-\frac{\gamma^{2}}{\nu} T C_{2}
$$

for some positive constants $C_{1}$ and $C_{2}$.

Let us estimate $\left\langle\widehat{T}_{j}^{(1)} \widehat{S} \widehat{T}_{j}^{(1)} u^{(0)}, u^{(0) *}\right\rangle$ for $j=2, \ldots, n-1$. We see from (5.14)

$$
\widehat{S} \widehat{T}_{j}^{(1)} u^{(0)}=-i \alpha_{0}\left(\begin{array}{c}
0 \\
\left(e^{-T \nu A}-1\right)^{-1} \int_{0}^{T} e^{-(T-s) \nu A} \cdot 1 d s \boldsymbol{e}_{j}^{\prime} \\
0
\end{array}\right)
$$


for $j=2, \ldots, n-1$. Therefore, we get

$$
\begin{aligned}
& \left\langle\widehat{T}_{j}^{(1)} \widehat{S} \widehat{T}_{j}^{(1)} u^{(0)}, u^{(0) *}\right\rangle \\
& =\alpha_{0}\left\langle\int_{0}^{T} \widehat{U}_{0}(T, s)\left(\begin{array}{c}
-\gamma^{2} \rho_{p} e^{-s \nu A}\left(e^{-T \nu A}-1\right)^{-1} \int_{0}^{T} e^{-\left(T-s_{1}\right) \nu A} \cdot 1 d s_{1} \\
0 \\
\frac{\widetilde{\nu}}{\rho_{p}} \partial_{x_{n}} e^{-s \nu A}\left(e^{-T \nu A}-1\right)^{-1} \int_{0}^{T} e^{-\left(T-s_{1}\right) \nu A} \cdot 1 d s_{1}
\end{array}\right) d s, u^{(0) *}\right\rangle .
\end{aligned}
$$

From (5.16) we can easily see that $\left\langle\widehat{T}_{j}^{(1)} \widehat{S} \widehat{T}_{j}^{(1)} u^{(0)}, u^{(0) *}\right\rangle$ is of order $\frac{\gamma^{2}}{\nu^{2}}$.

We finally consider $\widehat{\mu}_{11}^{(2)}$.

Proposition 5.15. There holds

$$
\widehat{\mu}_{11}^{(2)}=-\frac{\gamma^{2}}{\nu} T\left(K+O\left(\frac{1}{\gamma}\right)+O\left(\frac{1}{\nu}\right)+O\left(\frac{\nu+\widetilde{\nu}}{\gamma^{2}}\right)\right) .
$$

Proof of Proposition 5.15. We get

$$
\begin{aligned}
& \left\langle\widehat{T}_{11}^{(3)} u^{(0)}, u^{(0) *}\right\rangle \\
& =\left\langle\int_{0}^{T} \int_{0}^{s} \widehat{U}_{0}(T, s) \widehat{M}_{1}^{(1)}(s) \widehat{U}_{0}\left(s, s_{1}\right) i\left(\begin{array}{c}
\phi^{(0)} v_{p}^{1}\left(s_{1}\right)+\rho_{p} w^{(0), 1}\left(s_{1}\right) \\
\left(\alpha_{0}+v_{p}^{1}\left(s_{1}\right) \frac{1}{\gamma^{2}} w^{(0), 1}\left(s_{1}\right)\right) \boldsymbol{e}_{1}^{\prime} \\
-\frac{\widetilde{\nu}}{\gamma^{2} \rho_{p}} \partial_{x_{n}} w^{(0), 1}\left(s_{1}\right)
\end{array}\right) d s_{1} d s, u^{(0) *}\right\rangle,
\end{aligned}
$$

and

$$
\left\langle\widehat{T}_{1}^{(1)} \widehat{S} \widehat{T}_{1}^{(1)} u^{(0)}, u^{(0) *}\right\rangle=\left\langle\widehat{T}_{1}^{(1)} \widehat{S} \int_{0}^{T} \widehat{U}_{0}(T, s) i\left(\begin{array}{c}
\phi^{(0)} v_{p}^{1}(s)+\rho_{p} w^{(0), 1}(s) \\
\left(\alpha_{0}+v_{p}^{1}(s) \frac{1}{\gamma^{2}} w^{(0), 1}(s)\right) \boldsymbol{e}_{1}^{\prime} \\
-\frac{\widetilde{\nu}}{\gamma^{2} \rho_{p}} \partial_{x_{n}} w^{(0), 1}(s)
\end{array}\right) d s, u^{(0) *}\right\rangle
$$

To estimate the right-hand side of (5.17) and (5.18) it is convenient to transform the whole problem and consider newly obtained monodromy operator $\widetilde{U}_{\xi^{\prime}}(T)$

$$
\widehat{U}_{\xi^{\prime}}(T)=Q_{\gamma} \widetilde{U}_{\xi^{\prime}}(T) Q_{\gamma}^{-1}
$$

where

$$
Q_{\gamma}=\left(\begin{array}{rr}
\gamma & 0 \\
0 & I_{n}
\end{array}\right),
$$


which leads to the fact that spectrum of these operators is the same. Furthermore, we have

$$
\widehat{U}_{0}(T)=Q_{\gamma} \widetilde{U}_{0}(T) Q_{\gamma}^{-1}
$$

We set $\widetilde{u}=Q_{\gamma}^{-1} u$. Then $\widetilde{u}={ }^{T}(\widetilde{\phi}, \widetilde{w})=\widetilde{U}_{0}(t, s) \widetilde{u}_{0}$ is a solution of the problem

$$
\begin{gathered}
\partial_{t} \widetilde{\phi}+\gamma \partial_{x_{n}}\left(\rho_{p} \widetilde{w}^{n}\right)=0, \\
\partial_{t} \widetilde{w}^{\prime}-\frac{\nu}{\rho_{p}} \partial_{x_{n}}^{2} \widetilde{w}^{\prime}+\left(\partial_{x_{n}} v_{p}^{1}\right) \widetilde{w}^{n} \boldsymbol{e}_{1}^{\prime}+\frac{\nu}{\gamma \rho_{p}^{2}}\left(\partial_{x_{n}}^{2} v_{p}^{1}\right) \widetilde{\phi} \boldsymbol{e}_{1}^{\prime}=0, \\
\partial_{t} \widetilde{w}^{n}-\frac{\nu+\widetilde{\nu}}{\rho_{p}} \partial_{x_{n}}^{2} \widetilde{w}^{n}+\gamma \partial_{x_{n}}\left(\frac{\widetilde{P}^{\prime}\left(\rho_{p}\right)}{\gamma^{2} \rho_{p}} \widetilde{\phi}\right)=0, \\
\left.\widetilde{w}\right|_{x_{n}=0,1}=0,
\end{gathered}
$$

for $t>s \geq 0$, and

$$
\left.\widetilde{u}\right|_{t=s}=\widetilde{u}_{0}={ }^{T}\left(\widetilde{\phi}_{0}, \widetilde{w}_{0}\right) \text {. }
$$

One can show that

$$
\begin{gathered}
|\widetilde{\phi}(t)|_{2}+\left|\widetilde{w}^{n}(t)\right|_{2} \leq C\left(\left|\widetilde{\phi}_{0}\right|_{2}+\left|\widetilde{w}_{0}^{n}\right|_{2}\right), \\
\int_{0}^{t}\left|\widetilde{w}^{n}\right|_{2} d s \leq \int_{0}^{t}\left|\partial_{x_{n}} \widetilde{w}^{n}\right|_{2} d s \leq C \sqrt{t} \frac{1}{\sqrt{\nu+\widetilde{\nu}}}\left(\left|\widetilde{\phi}_{0}\right|_{2}+\left|\widetilde{w}_{0}^{n}\right|_{2}\right), \\
|\widetilde{\phi}(t)|_{H^{1}} \leq C\left|\widetilde{\phi}_{0}\right|_{H^{1}}+C\left(\frac{\gamma}{\nu+\widetilde{\nu}}+\frac{1}{\gamma^{2}}+\sqrt{t} \frac{1}{\gamma \sqrt{\nu+\widetilde{\nu}}}\right)\left(\left|\widetilde{\phi}_{0}\right|_{2}+\left|\widetilde{w}_{0}^{n}\right|_{2}\right), \\
|\widetilde{S}|_{L(X)}=\left|Q_{\gamma}^{-1} \widehat{S} Q_{\gamma}\right|_{L(X)} \leq C .
\end{gathered}
$$

Furthermore, (5.17) can be written as

$$
\begin{aligned}
& \left\langle\widehat{T}_{11}^{(3)} u^{(0)}, u^{(0) *}\right\rangle \\
& =\left\langle\int_{0}^{T} \int_{0}^{s} \widetilde{U}_{0}(T, s) \widetilde{M}_{1}^{(1)}(s) \widetilde{U}_{0}\left(s, s_{1}\right) i\left(\begin{array}{c}
\phi^{(0)} v_{p}^{1}\left(s_{1}\right)+\rho_{p} w^{(0), 1}\left(s_{1}\right) \\
\gamma\left(\alpha_{0}+v_{p}^{1}\left(s_{1}\right) \frac{1}{\gamma^{2}} w^{(0), 1}\left(s_{1}\right)\right) \boldsymbol{e}_{1}^{\prime} \\
-\frac{\widetilde{\nu}}{\gamma \rho_{p}} \partial_{x_{n}} w^{(0), 1}\left(s_{1}\right)
\end{array}\right) d s_{1} d s, u^{(0) *}\right\rangle,
\end{aligned}
$$

where

$$
\widetilde{M}_{1}^{(1)}(s)=Q_{\gamma}^{-1} \widehat{M}_{1}^{(1)}(s) Q_{\gamma}=\left(\begin{array}{ccc}
i v_{p}^{1}(s) & i \gamma \rho_{p} \boldsymbol{e}_{1}^{\prime} & 0 \\
i \gamma \frac{\widetilde{P}^{\prime}\left(\rho_{p}\right)}{\gamma^{2} \rho_{p}} \boldsymbol{e}_{1}^{\prime} & i v_{p}^{1}(s) I_{n-1} & -i \frac{\tilde{\nu}}{\rho_{p}} \partial_{x_{n}} \boldsymbol{e}_{1}^{\prime} \\
0 & -i \frac{\tilde{\nu}}{\rho_{p}} \partial_{x_{n}}{ }^{T} \boldsymbol{e}_{1}^{\prime} & i v_{p}^{1}(s)
\end{array}\right)
$$


From (5.15) and (5.20) it is easy to see that for $j \neq 1$

$$
\begin{aligned}
& \left\langle\widehat{T}_{11}^{(3)} u^{(0)}, u^{(0) *}\right\rangle=\left\langle\widehat{T}_{j j}^{(3)} u^{(0)}, u^{(0) *}\right\rangle \\
& \quad+\left\langle\int_{0}^{T} \int_{0}^{s} \widetilde{U}_{0}(T, s) \widetilde{M}_{1}^{(1)}(s) \widetilde{U}_{0}\left(s, s_{1}\right) i\left(\begin{array}{c}
\phi^{(0)} v_{p}^{1}\left(s_{1}\right)+\rho_{p} w^{(0), 1}\left(s_{1}\right) \\
v_{p}^{1}\left(s_{1}\right) \frac{1}{\gamma} w^{(0), 1}\left(s_{1}\right) \boldsymbol{e}_{1}^{\prime} \\
-\frac{\widetilde{\nu}}{\gamma \rho_{p}} \partial_{x_{n}} w^{(0), 1}\left(s_{1}\right)
\end{array}\right) d s_{1} d s, u^{(0) *}\right\rangle .
\end{aligned}
$$

Using the estimates (5.19) we get

$$
\left|\left\langle\widehat{T}_{11}^{(3)} u^{(0)}, u^{(0) *}\right\rangle-\left\langle\widehat{T}_{j j}^{(3)} u^{(0)}, u^{(0) *}\right\rangle\right| \leq C \frac{\gamma^{2}}{\nu}\left(\frac{1}{\gamma}+\frac{\nu+\widetilde{\nu}}{\gamma^{2}}\right) .
$$

Similarly, we can estimate the right-hand side of (5.18) as

$$
\left|\left\langle\widehat{T}_{1}^{(1)} \widehat{S} \widehat{T}_{1}^{(1)} u^{(0)}, u^{(0) *}\right\rangle\right| \leq C \frac{\gamma^{2}}{\nu}\left(\frac{1}{\gamma}+\frac{1}{\nu}+\frac{\nu+\widetilde{\nu}}{\gamma^{2}}\right)
$$

We thus obtain

$$
\widehat{\mu}_{11}^{(2)}=-\frac{\gamma^{2}}{\nu} T\left(K+O\left(\frac{1}{\gamma}\right)+O\left(\frac{1}{\nu}\right)+O\left(\frac{\nu+\widetilde{\nu}}{\gamma^{2}}\right)\right) .
$$

This completes the proof.

We now turn to the proof of Theorem 5.11. One can see from Propositions 5.13, 5.14 and 5.15 that $\widehat{\mu}_{j j}^{(2)}<0$ if $\frac{\nu+\tilde{\nu}}{\gamma^{2}}, \frac{1}{\nu}$ and $\frac{1}{\gamma}$ are sufficiently small. Therefore, the desired asymptotic of $\widehat{\mu}\left(\xi^{\prime}\right)$ is obtained from Propositions 5.12-5.15. This completes the proof of Theorem 5.11.

Theorem 5.16. There exist constants $\nu_{0}>0$ and $\gamma_{0}>0$ such that if $\nu \geq \nu_{0}$ and $\gamma^{2} /(\nu+\widetilde{\nu}) \geq$ $\gamma_{0}^{2}$ then the following statements hold true:

(i) Let $\widehat{\Pi}\left(\xi^{\prime}\right)$ be the eigen-projection associated with $\widehat{\mu}\left(\xi^{\prime}\right)$. Then there exists a positive number $r_{0}$ such that for any $\xi^{\prime}$ with $\left|\xi^{\prime}\right| \leq r_{0}$ the projection $\widehat{\Pi}\left(\xi^{\prime}\right)$ is written in the form

$$
\widehat{\Pi}\left(\xi^{\prime}\right)=\widehat{\Pi}^{(0)}+\widehat{\Pi}^{(1)}\left(\xi^{\prime}\right),
$$

where

$$
\widehat{\Pi}^{(1)}\left(\xi^{\prime}\right)=\sum_{j=1}^{n-1} \xi_{j} \widehat{\Pi}_{j}^{(1)}+\widehat{\Pi}^{(2)}\left(\xi^{\prime}\right) \text { and } \widehat{\Pi}_{j}^{(1)}=\widehat{\Pi}^{(0)} \widehat{T}_{j}^{(1)} \widehat{S}+\widehat{S}_{j}^{(1)} \widehat{\Pi}^{(0)} .
$$

Furthermore, we have estimates

$$
\begin{gathered}
\left|\widehat{\Pi}_{j}^{(1)} u_{0}\right|_{H^{1} \times L^{2}} \leq C\left|u_{0}\right|_{H^{1} \times L^{2}}, \\
\left|\widehat{\Pi}^{(2)}\left(\xi^{\prime}\right) u_{0}\right|_{H^{1} \times L^{2}} \leq C\left|\xi^{\prime}\right|^{2}\left|u_{0}\right|_{H^{1} \times L^{2}} .
\end{gathered}
$$


(ii) There exists a positive number $r_{0}$ such that for any $\xi^{\prime}$ with $\left|\xi^{\prime}\right| \leq r_{0}$ the spectral radius of $\widehat{U}_{\xi^{\prime}}(T)$ on $\left(I-\widehat{\Pi}\left(\xi^{\prime}\right)\right) X$ satisfies estimate

$$
r\left(\left.\widehat{U}_{\xi^{\prime}}(T)\right|_{\left(I-\widehat{\Pi}\left(\xi^{\prime}\right)\right) X}\right) \leq \delta_{0}<1
$$

To prove Theorem 5.16 and Theorem 5.1 we use the following estimates.

Lemma 5.17. There exists a constant $\gamma_{0}>0$ such that if $\gamma \geq \gamma_{0}$ then for $0<t-s \leq 2 T$ and $\left|\xi^{\prime}\right|<1$ hold true the following estimates:

$$
\begin{gathered}
\left|\widehat{U}_{\xi^{\prime}}(t, s) \widehat{u}_{0}\right|_{H^{1} \times L^{2}} \leq C\left|\widehat{u}_{0}\right|_{H^{1} \times L^{2}}, \\
\left|\partial_{x_{n}} \widetilde{Q} \widehat{U}_{\xi^{\prime}}(t, s) \widehat{u}_{0}\right|_{L^{2}} \leq C(t-s)^{-\frac{1}{2}}\left|\widehat{u}_{0}\right|_{H^{1} \times L^{2}} .
\end{gathered}
$$

Proof of Lemma 5.17. Since $\widehat{U}_{\xi^{\prime}}(t, s)=\widehat{U}_{0}(t, s)+\widehat{V}_{\xi^{\prime}}(t, s)$, we see from $(5.11)$

$$
\widehat{U}_{\xi^{\prime}}(t, s) u_{0}=\widehat{U}_{0}(t, s) u_{0}-\int_{s}^{t} \widehat{U}_{0}(t, z)\left(\widehat{M}_{\xi^{\prime}}-\widehat{M}_{0}\right)(z) \widehat{U}_{\xi^{\prime}}(z, s) u_{0} d z
$$

First let us estimate $\left|\widehat{U}_{\xi^{\prime}}(t, s) u_{0}\right|_{H^{1} \times L^{2}}$. We have

$$
\left|\widehat{U}_{\xi^{\prime}}(t, s) u_{0}\right|_{H^{1} \times L^{2}} \leq\left|\widehat{U}_{0}(t, s) u_{0}\right|_{H^{1} \times L^{2}}+\int_{s}^{t}\left|\widehat{U}_{0}(t, z)\left(\widehat{M}_{\xi^{\prime}}-\widehat{M}_{0}\right)(z) \widehat{U}_{\xi^{\prime}}(z, s) u_{0}\right|_{H^{1} \times L^{2}} d z .
$$

Using estimate from Lemma 5.9 we have

$$
\begin{aligned}
& \left|\widehat{U}_{\xi^{\prime}}(t, s) u_{0}\right|_{H^{1} \times L^{2}} \leq C\left|u_{0}\right|_{H^{1} \times L^{2}}+C \int_{s}^{t}\left|\left(\widehat{M}_{\xi^{\prime}}-\widehat{M}_{0}\right)(z) \widehat{U}_{\xi^{\prime}}(z, s) u_{0}\right|_{H^{1} \times L^{2}} d z \\
& \quad \leq C\left|u_{0}\right|_{H^{1} \times L^{2}}+C \int_{s}^{t}\left|\xi^{\prime}\right|\left(\left|\widehat{U}_{\xi^{\prime}}(z, s) u_{0}\right|_{H^{1} \times L^{2}}+\left|\partial_{x_{n}} \widetilde{Q} \widehat{U}_{\xi^{\prime}}(z, s) u_{0}\right|_{L^{2}}\right) d z .
\end{aligned}
$$

Using Gronwall inequality we obtain

$$
\left|\widehat{U}_{\xi^{\prime}}(t, s) u_{0}\right|_{H^{1} \times L^{2}} \leq C e^{c_{1}(t-s)}\left(\left|u_{0}\right|_{H^{1} \times L^{2}}+\int_{s}^{t}\left|\partial_{x_{n}} \widetilde{Q} \widehat{U}_{\xi^{\prime}}(z, s) u_{0}\right|_{2} d z\right) .
$$

Now we estimate $\left|\partial_{x_{n}} \widetilde{Q} \widehat{U}_{\xi^{\prime}}(t, s) u_{0}\right|_{2}$ analogously to above estimates by using Lemma 5.9 and (5.26), to obtain

$$
\left|\partial_{x_{n}} \widetilde{Q} \widehat{U}_{\xi^{\prime}}(t, s) u_{0}\right|_{2} \leq C(t-s)^{-\frac{1}{2}}\left|u_{0}\right|_{H^{1} \times L^{2}},
$$

for $0<t-s \leq 2 T$. This together with (5.26) concludes the proof.

Proof of Theorem 5.16. Expansion of $\widehat{\Pi}\left(\xi^{\prime}\right)$ and (5.23) are obtained using results in [7]. Estimate (5.22) is obtained as follows. If $j \neq 1$, then 


$$
\begin{gathered}
\widehat{\Pi}^{(0)} \widehat{T}_{j}^{(1)} \widehat{S} u_{0}=\left[Q_{0} \widehat{T}_{j}^{(1)} \widehat{S} u_{0}\right] u^{(0)}=\int_{0}^{1} \int_{0}^{T} Q_{0} \widehat{U}_{0}(T, s) \widehat{M}_{j}^{(1)}(s) \widehat{U}_{0}(s, 0) \widehat{S} u_{0} d s d x_{n} u^{(0)} \\
=\int_{0}^{T} \int_{0}^{1} i \gamma^{2} \rho_{p} Q_{j} \widehat{U}_{0}(s, 0) \widehat{S} u_{0} d x_{n} d s u^{(0)}=\int_{0}^{T} \int_{0}^{1} i \gamma^{2} \rho_{p} e^{-s \nu A}\left(e^{-T \nu A}-1\right)^{-1} w_{0}^{j} d x_{n} d s u^{(0)},
\end{gathered}
$$

and therefore,

$$
\left|\widehat{\Pi}^{(0)} \widehat{T}_{j}^{(1)} \widehat{S} u_{0}\right|_{H^{1} \times L^{2}} \leq C \frac{\gamma^{2}}{\nu}\left|w_{0}^{j}\right|_{2} \text { for } j \neq 1
$$

If $j=1$, then

$$
\widehat{\Pi}^{(0)} \widehat{T}_{1}^{(1)} \widehat{S} u_{0}=\int_{0}^{T} \int_{0}^{1} i v_{p}^{1} Q_{0} \widehat{U}_{0}(s, 0) \widehat{S} u_{0}+i \gamma^{2} \rho_{p} Q_{1} \widehat{U}_{0}(s, 0) \widehat{S} u_{0} d x_{n} d s u^{(0)} .
$$

Therefore, we get

$$
\left|\widehat{\Pi}^{(0)} \widehat{T}_{1}^{(1)} \widehat{S} u_{0}\right|_{H^{1} \times L^{2}} \leq C\left(1+\gamma^{2}\right)\left|u_{0}\right|_{H^{1} \times L^{2}} .
$$

We next estimate $\widehat{S} \widehat{T}_{j}^{(1)} \widehat{\Pi}^{(0)} u_{0}$. If $j \neq 1$, then

$$
\begin{gathered}
\widehat{S} \widehat{T}_{j}^{(1)} \widehat{\Pi}^{(0)} u_{0}=\widehat{S} \widehat{T}_{j}^{(1)}\left[\phi_{0}\right] u^{(0)}=\left[\phi_{0}\right] \widehat{S} \int_{0}^{T} \widehat{U}_{0}(T, s) \widehat{M}_{j}^{(1)}(s) \widehat{U}_{0}(s, 0) u^{(0)} d s \\
=\left[\phi_{0}\right] \widehat{S} \int_{0}^{T} \widehat{U}_{0}(T, s) \widehat{M}_{j}^{(1)}(s)\left(\begin{array}{c}
\phi^{(0)} \\
\frac{1}{\gamma^{2}} w^{(0), 1}\left(x_{n}, t\right) \boldsymbol{e}_{1}^{\prime} \\
0
\end{array}\right) d s \\
=\left[\phi_{0}\right] \widehat{S} \int_{0}^{T} \widehat{U}_{0}(T, s)\left(\begin{array}{c}
0 \\
i \alpha_{0} \boldsymbol{e}_{j}^{\prime} \\
0
\end{array}\right) d s=i\left[\phi_{0}\right] \int_{0}^{T}\left(e^{-T \nu A}-1\right)^{-1} e^{-(T-s) \nu A} d s\left(\begin{array}{c}
0 \\
\alpha_{0} \boldsymbol{e}_{j}^{\prime} \\
0
\end{array}\right) .
\end{gathered}
$$

Therefore, we have

$$
\left|\widehat{S} \widehat{T}_{j}^{(1)} \widehat{\Pi}^{(0)} u_{0}\right|_{H^{1} \times L^{2}} \leq \frac{C}{\nu}\left|\phi_{0}\right|_{1} \text { for } j \neq 1
$$

In case $j=1$,

$$
\widehat{S} \widehat{T}_{1}^{(1)} \widehat{\Pi}^{(0)} u_{0}=i\left[\phi_{0}\right] \widehat{S} \int_{0}^{T} \widehat{U}_{0}(T, s)\left(\begin{array}{c}
\phi^{(0)} v_{p}^{1}+\rho_{p} w^{(0), 1}\left(x_{n}, t\right) \\
\left(\alpha_{0}+v_{p}^{1} \frac{1}{\gamma^{2}} w^{(0), 1}\left(x_{n}, t\right)\right) \boldsymbol{e}_{1}^{\prime} \\
-\frac{\widetilde{\nu}}{\gamma^{2} \rho_{p}} \partial_{x_{n}} w^{(0), 1}\left(x_{n}, t\right)
\end{array}\right) d s
$$

Therefore, we get following estimate

$$
\left|\widehat{S} \widehat{T}_{1}^{(1)} \widehat{\Pi}^{(0)} u_{0}\right|_{H^{1} \times L^{2}} \leq C\left(1+\frac{1}{\gamma^{2}}+\frac{\widetilde{\nu}}{\gamma^{2}}\right)\left|\phi_{0}\right|_{1} .
$$


Thus we proved (5.22). From (5.22) and (5.23) we immediately get following estimate

$$
\left|\left(I-\widehat{\Pi}\left(\xi^{\prime}\right)\right) u_{0}\right|_{H^{1} \times L^{2}} \leq C\left[1+\left|\xi^{\prime}\right|\left(1+\gamma^{2}\right)+O\left(\left|\xi^{\prime}\right|^{2}\right)\right]\left|u_{0}\right|_{H^{1} \times L^{2}}
$$

Now we concentrate on proving the estimate on spectral radius. From (4.38) we see that there exist constants $\nu_{0}>0$ and $\gamma_{0}>0$ such that if $\nu \geq \nu_{0}$ and $\gamma^{2} /(\nu+\widetilde{\nu}) \geq \gamma_{0}^{2}$ then we have

$$
\left|\widehat{U}_{\xi^{\prime}}(t, s) u_{0}\right|_{H^{1}}^{2} \leq C e^{-c_{0}\left|\xi^{\prime}\right|^{2}(t-s-4 T)} E_{3}\left(\widehat{u}_{s}\left(\xi^{\prime}, \cdot, s+4 T\right)\right),
$$

for $\left|\xi^{\prime}\right| \leq R_{0}$ and $t-s \geq 4 T, s \geq 0$. Using Lemma 4.4 on $E_{3}\left(\widehat{u}_{s}\left(\xi^{\prime}, \cdot, s+4 T\right)\right)$ we get following estimate,

$$
\left|\widehat{U}_{\xi^{\prime}}(t, s) u_{0}\right|_{H^{1} \times L^{2}}^{2} \leq C\left|u_{0}\right|_{H^{1} \times L^{2}}^{2} .
$$

Using (5.21), (5.22), (5.23) and (5.25) we get

$$
\begin{gathered}
\left|\left\{\left(I-\widehat{\Pi}\left(\xi^{\prime}\right)\right) \widehat{U}_{\xi^{\prime}}(T)\right\}^{m}\right|_{L(X)}=\left|\left(I-\widehat{\Pi}\left(\xi^{\prime}\right)\right) \widehat{U}_{\xi^{\prime}}(m T, 0)\right|_{L(X)} \\
\leq\left|\widehat{\Pi}_{c}^{(0)} \widehat{U}_{\xi^{\prime}}(m T, 0)\right|_{L(X)}+\left|\widehat{\Pi}^{(1)}\left(\xi^{\prime}\right) \widehat{U}_{\xi^{\prime}}(m T, 0)\right|_{L(X)} \leq\left|\widehat{\Pi}_{c}^{(0)} \widehat{U}_{0}(m T, 0)\right|_{L(X)} \\
+\left|\int_{0}^{m T} \widehat{\Pi}_{c}^{(0)} \widehat{U}_{0}(m T, z)\left(\widehat{M}_{\xi^{\prime}}-\widehat{M}_{0}\right)(z) \widehat{U}_{\xi^{\prime}}(z, 0) d z\right|_{L(X)}+C\left|\xi^{\prime}\right|\left|\widehat{U}_{\xi^{\prime}}(m T, 0)\right|_{L(X)}
\end{gathered}
$$

Combining this with (5.7) and (5.28) we obtain

$$
\left|\left\{\left(I-\widehat{\Pi}\left(\xi^{\prime}\right)\right) \widehat{U}_{\xi^{\prime}}(T)\right\}^{m}\right|_{L(X)} \leq C e^{-d m T}+C\left|\xi^{\prime}\right|
$$

for any $m \in N$ and some $d>0$. Let us compute a spectral radius of $\widehat{U}_{\xi^{\prime}}(T, 0)$ on complementary space $\left(I-\widehat{\Pi}\left(\xi^{\prime}\right)\right) X$,

$$
\begin{aligned}
& r\left(\left.\widehat{U}_{\xi^{\prime}}(T)\right|_{\left(I-\widehat{\Pi}\left(\xi^{\prime}\right)\right) X}\right)=r\left(\left(I-\widehat{\Pi}\left(\xi^{\prime}\right)\right) \widehat{U}_{\xi^{\prime}}(T)\left(I-\widehat{\Pi}\left(\xi^{\prime}\right)\right)\right) \\
\leq & \left|\left(\left(I-\widehat{\Pi}\left(\xi^{\prime}\right)\right) \widehat{U}_{\xi^{\prime}}(T)\left(I-\widehat{\Pi}\left(\xi^{\prime}\right)\right)\right)^{m}\right|_{L(X)}^{\frac{1}{m}}, \text { for any } m \in N
\end{aligned}
$$

Fix $m_{0} \in N$ large and $r_{0}>0$ small such that for $\left|\xi^{\prime}\right| \leq r_{0}$ it holds for (5.29) that

$$
C e^{-d m T}+C\left|\xi^{\prime}\right| \leq e^{-T}
$$

Then we immediately see that

$$
r\left(\left.\widehat{U}_{\xi^{\prime}}(T)\right|_{\left(I-\widehat{\Pi}\left(\xi^{\prime}\right)\right) X}\right) \leq e^{-\frac{T}{m_{0}}}<1
$$

for all $\left|\xi^{\prime}\right| \leq r_{0}$

Proof of Theorem 5.1. Theorem 5.1 now follows from Lemma 5.9, Theorem 5.11, 5.16 and Lemma 5.17 in the following way. Let us fix positive numbers $\nu_{0}, \gamma_{0}$ and $r_{0}<1$ such 
that if $\nu \geq \nu_{0}$ and $\gamma^{2} /(\nu+\tilde{\nu}) \geq \gamma_{0}^{2}$ then for each $\xi^{\prime}$ with $\left|\xi^{\prime}\right| \leq r_{0}$ all above mentioned statements 5.9-5.17 holds true. We also assume that $t-s \geq 4 T$ and $k, l=0,1$.

As an immediate consequence of Theorem 5.11 we have following decomposition,

$$
\widehat{U}_{\xi^{\prime}}(T)=\widehat{U}_{\xi^{\prime}}(T) \widehat{\Pi}\left(\xi^{\prime}\right)+\widehat{U}_{\xi^{\prime}}(T)\left(I-\widehat{\Pi}\left(\xi^{\prime}\right)\right)=\widehat{\mu}\left(\xi^{\prime}\right) \widehat{\Pi}\left(\xi^{\prime}\right)+\widehat{U}_{\xi^{\prime}}(T)\left(I-\widehat{\Pi}\left(\xi^{\prime}\right)\right),
$$

where

$$
\widehat{\mu}\left(\xi^{\prime}\right)=1+\lambda_{0}\left(\xi^{\prime}\right) T=e^{\lambda_{0}\left(\xi^{\prime}\right) T} .
$$

We further write $\mathscr{V}_{0}(t, s) u_{0}$ as

$$
\begin{aligned}
\mathscr{V}_{0}(t, s) u_{0}= & \sigma_{t, s} u^{(0)}(t)+\mathscr{U}_{1}^{(0)}(t, s) u_{0}+\mathscr{U}_{2}^{(0)}(t, s) u_{0} \\
& +\mathscr{U}_{3}^{(0)}(t, s) u_{0}+\mathscr{R}^{(0)}(t, s) u_{0},
\end{aligned}
$$

where

$$
\begin{aligned}
\sigma_{t, s} u^{(0)}(t) & =\mathscr{F}^{-1}\left(e^{-\left(i \kappa_{0} \xi_{1}+\kappa_{1}\left|\xi^{\prime}\right|^{2}\right)(t-s)} \widehat{\Pi}^{(0)}(t) \widehat{u}_{0}\right), \\
\mathscr{U}_{1}^{(0)}(t, s) u_{0} & =\mathscr{F}^{-1}\left(\left(\chi^{(0)}-1\right) e^{-\left(i \kappa_{0} \xi_{1}+\kappa_{1}\left|\xi^{\prime}\right|^{2}\right)(t-s)} \widehat{\Pi}^{(0)}(t) \widehat{u}_{0}\right), \\
\mathscr{U}_{2}^{(0)}(t, s) u_{0} & =\mathscr{F}^{-1}\left(\chi^{(0)}\left(e^{\lambda_{0}\left(\xi^{\prime}\right)(t-s)}-e^{-\left(i \kappa_{0} \xi_{1}+\kappa_{1}\left|\xi^{\prime}\right|^{2}\right)(t-s)}\right) \widehat{\Pi}^{(0)}(t) \widehat{u}_{0}\right), \\
\mathscr{U}_{3}^{(0)}(t, s) u_{0} & =\mathscr{F}^{-1}\left(\chi^{(0)}\left(\widehat{U}_{\xi^{\prime}}\left(t, t-\tau_{1}\right) \widehat{\Pi}\left(\xi^{\prime}\right) \widehat{U}_{\xi^{\prime}}(T)^{m} \widehat{U}_{\xi^{\prime}}\left(s+\tau_{2}, s\right)-e^{\lambda_{0}\left(\xi^{\prime}\right)(t-s)} \widehat{\Pi}^{(0)}(t)\right) \widehat{u}_{0}\right), \\
\mathscr{R}^{(0)}(t, s) u_{0} & =\mathscr{F}^{-1}\left(\chi^{(0)} \widehat{U}_{\xi^{\prime}}\left(t, t-\tau_{1}\right)\left(I-\widehat{\Pi}\left(\xi^{\prime}\right)\right) \widehat{U}_{\xi^{\prime}}(T)^{m} \widehat{U}_{\xi^{\prime}}\left(s+\tau_{2}, s\right) \widehat{u}_{0}\right) .
\end{aligned}
$$

Here $\mathscr{U}_{3}^{(0)}(t, s) u_{0}$ and $\mathscr{R}^{(0)}(t, s) \widehat{u}_{0}$ are given as follows. Since

$$
\widehat{U}_{\xi^{\prime}}(t+T, s+T)=\widehat{U}_{\xi^{\prime}}(t, s),
$$

if $\tau_{1}, \tau_{2}, m$ is defined in such a way that $t-s=\tau_{1}+m T+\tau_{2}$, where $t-\tau_{1}, s+\tau_{2}$ are integer multiples of $T$ and $\tau_{1}, \tau_{2} \in[T, 2 T)$, we have

$$
\begin{aligned}
& \mathscr{F}^{-1}\left(\chi^{(0)}\left(\widehat{U}_{\xi^{\prime}}(t, s)-e^{\lambda_{0}\left(\xi^{\prime}\right)(t-s)} \widehat{\Pi}^{(0)}(t)\right) \widehat{u}_{0}\right) \\
& =\mathscr{F}^{-1}\left(\chi^{(0)}\left(\widehat{U}_{\xi^{\prime}}\left(t, t-\tau_{1}\right) \widehat{U}_{\xi^{\prime}}(T)^{m} \widehat{U}_{\xi^{\prime}}\left(s+\tau_{2}, s\right)-e^{\lambda_{0}\left(\xi^{\prime}\right)(t-s)} \widehat{\Pi}^{(0)}(t)\right) \widehat{u}_{0}\right) \\
& =\mathscr{F}^{-1}\left(\chi^{(0)}\left(\widehat{U}_{\xi^{\prime}}\left(t, t-\tau_{1}\right) \widehat{\Pi}\left(\xi^{\prime}\right) \widehat{U}_{\xi^{\prime}}(T)^{m} \widehat{U}_{\xi^{\prime}}\left(s+\tau_{2}, s\right)-e^{\lambda_{0}\left(\xi^{\prime}\right)(t-s)} \widehat{\Pi}^{(0)}(t)\right) \widehat{u}_{0}\right) \\
& +\mathscr{F}^{-1}\left(\chi^{(0)} \widehat{U}_{\xi^{\prime}}\left(t, t-\tau_{1}\right)\left(I-\widehat{\Pi}\left(\xi^{\prime}\right)\right) \widehat{U}_{\xi^{\prime}}(T)^{m} \widehat{U}_{\xi^{\prime}}\left(s+\tau_{2}, s\right) \widehat{u}_{0}\right)=\mathscr{U}_{3}^{(0)}(t, s) u_{0}+\mathscr{R}^{(0)}(t, s) u_{0} .
\end{aligned}
$$

As for $\left\|\partial_{x^{\prime}}^{k} \partial_{x_{n}}^{l} \mathscr{U}_{3}^{(0)}(t, s) u_{0}\right\|_{2}^{2}$, we have following estimates. Let us first introduce estimates in $x_{n}$. Let

$$
I\left(\xi^{\prime} ; t, s\right)=\widehat{U}_{\xi^{\prime}}\left(t, t-\tau_{1}\right) \widehat{\Pi}\left(\xi^{\prime}\right) \widehat{U}_{\xi^{\prime}}(T)^{m} \widehat{U}_{\xi^{\prime}}\left(s+\tau_{2}, s\right) \widehat{u}_{0}-e^{\lambda_{0}\left(\xi^{\prime}\right)(t-s)} \widehat{\Pi}^{(0)}(t) \widehat{u}_{0} .
$$


Since $\widehat{\Pi}\left(\xi^{\prime}\right) \widehat{U}_{\xi^{\prime}}(T)=e^{\lambda_{0}\left(\xi^{\prime}\right) T} \widehat{\Pi}\left(\xi^{\prime}\right)$, we have

$$
\begin{gathered}
I\left(\xi^{\prime} ; t, s\right)=\widehat{U}_{\xi^{\prime}}\left(t, t-\tau_{1}\right) e^{\lambda_{0}\left(\xi^{\prime}\right) m T} \widehat{\Pi}\left(\xi^{\prime}\right) \widehat{U}_{\xi^{\prime}}\left(s+\tau_{2}, s\right) \widehat{u}_{0}-e^{\lambda_{0}\left(\xi^{\prime}\right)(t-s)} \widehat{\Pi}^{(0)}(t) \widehat{u}_{0} \\
=I_{1}\left(\xi^{\prime} ; t, s\right)+I_{2}\left(\xi^{\prime} ; t, s\right),
\end{gathered}
$$

where

$$
\begin{gathered}
I_{1}\left(\xi^{\prime} ; t, s\right)=\left(\widehat{U}_{\xi^{\prime}}\left(t, t-\tau_{1}\right) e^{\lambda_{0}\left(\xi^{\prime}\right) m T} \widehat{\Pi}^{(0)} \widehat{U}_{\xi^{\prime}}\left(s+\tau_{2}, s\right)-e^{\lambda_{0}\left(\xi^{\prime}\right)(t-s)} \widehat{\Pi}^{(0)}(t)\right) \widehat{u}_{0}, \\
I_{2}\left(\xi^{\prime} ; t, s\right)=\widehat{U}_{\xi^{\prime}}\left(t, t-\tau_{1}\right) e^{\lambda_{0}\left(\xi^{\prime}\right) m T} \widehat{\Pi}^{(1)}\left(\xi^{\prime}\right) \widehat{U}_{\xi^{\prime}}\left(s+\tau_{2}, s\right) \widehat{u}_{0} .
\end{gathered}
$$

Since $\left|\left(M_{\xi^{\prime}}-M_{0}\right)(z) u\right|_{H^{1} \times L^{2}} \leq C\left|\xi^{\prime}\right|\left(|u|_{H^{1} \times L^{2}}+\left|\partial_{x_{n}} w\right|_{2}\right)$, applying (5.25) for $\widehat{U}_{\xi^{\prime}}\left(t, t-\tau_{1}\right)$ and $\widehat{U}_{\xi^{\prime}}\left(s+\tau_{2}, s\right)$, we have

$$
\begin{gathered}
\left|I_{1}\left(\xi^{\prime} ; t, s\right)\right|_{H^{1}} \leq\left|\left(\widehat{U}_{0}\left(t, t-\tau_{1}\right) e^{\lambda_{0}\left(\xi^{\prime}\right) m T} \widehat{\Pi}^{(0)} \widehat{U}_{0}\left(s+\tau_{2}, s\right)-e^{\lambda_{0}\left(\xi^{\prime}\right)(t-s)} \widehat{\Pi}^{(0)}(t)\right) \widehat{u}_{0}\right|_{H^{1}} \\
+C\left|e^{\lambda_{0}\left(\xi^{\prime}\right) m T}\right|\left|\xi^{\prime}\right|\left|\widehat{u}_{0}\right|_{H^{1} \times L^{2}}
\end{gathered}
$$

Let us consider the first term on the right of (5.31). By Lemma 4.6 (ii) we have

$$
\widehat{\Pi}^{(0)} \widehat{U}_{0}\left(s+\tau_{2}, s\right) \widehat{u}_{0}=\left[Q_{0} \widehat{U}_{0}\left(s+\tau_{2}, s\right) \widehat{u}_{0}\right] u^{(0)}=\left[\widehat{\phi}_{0}\right] u^{(0)} .
$$

Since $t-\tau_{1}=m T+s+\tau_{2}=m^{\prime} T$ for some $m^{\prime} \in \mathbb{N}$, we see from Lemma 5.2

$$
\widehat{U}_{0}\left(t, t-\tau_{1}\right) u^{(0)}=\widehat{U}_{0}\left(\tau_{1}, 0\right) u^{(0)}=u^{(0)}\left(\tau_{1}\right) .
$$

On the other hand, since $u^{(0)}(t)=u^{(0)}\left(\tau_{1}\right)$, we have

$$
e^{\lambda_{0}\left(\xi^{\prime}\right)(t-s)} \widehat{\Pi}^{(0)}(t) \widehat{u}_{0}=e^{\lambda_{0}\left(\xi^{\prime}\right)(t-s)}\left[\widehat{\phi}_{0}\right] u^{(0)}(t)=e^{\lambda_{0}\left(\xi^{\prime}\right)(t-s)}\left[\widehat{\phi}_{0}\right] u^{(0)}\left(\tau_{1}\right) .
$$

We thus obtain

$$
\begin{gathered}
\left(\widehat{U}_{0}\left(t, t-\tau_{1}\right) e^{\lambda_{0}\left(\xi^{\prime}\right) m T} \widehat{\Pi}^{(0)} \widehat{U}_{0}\left(s+\tau_{2}, s\right)-e^{\lambda_{0}\left(\xi^{\prime}\right)(t-s)} \widehat{\Pi}^{(0)}(t)\right) \widehat{u}_{0} \\
=\left(e^{\lambda_{0}\left(\xi^{\prime}\right) m T}-e^{\lambda_{0}\left(\xi^{\prime}\right)(t-s)}\right)\left[\widehat{\phi}_{0}\right] u^{(0)}(t)
\end{gathered}
$$

Since

$$
\begin{aligned}
e^{\lambda_{0}\left(\xi^{\prime}\right) m T}-e^{\lambda_{0}\left(\xi^{\prime}\right)(t-s)} & =e^{\lambda_{0}\left(\xi^{\prime}\right) m T}\left(1-e^{\lambda_{0}\left(\xi^{\prime}\right)\left(\tau_{1}+\tau_{2}\right)}\right) \\
& =e^{\lambda_{0}\left(\xi^{\prime}\right) m T} \int_{0}^{1} e^{\theta \lambda_{0}\left(\xi^{\prime}\right)\left(\tau_{1}+\tau_{2}\right)} d \theta \lambda_{0}\left(\xi^{\prime}\right)\left(\tau_{1}+\tau_{2}\right),
\end{aligned}
$$

we see from (5.31) and (5.32)

$$
\left|I_{1}\left(\xi^{\prime} ; t, s\right)\right|_{H^{1}} \leq C\left|\xi^{\prime}\right|\left|e^{\lambda_{0}\left(\xi^{\prime}\right) m T}\right|\left(\left|\widehat{\phi}_{0}\right|_{1}+\left|\widehat{u}_{0}\right|_{H^{1} \times L^{2}}\right) .
$$

Using estimates (5.22), (5.23) and (5.24) we obtain

$$
\left|I_{2}\left(\xi^{\prime} ; t, s\right)\right|_{H^{1}} \leq C\left|\xi^{\prime}\right|\left|e^{\lambda_{0}\left(\xi^{\prime}\right) m T}\right|\left|\widehat{u}_{0}\right|_{H^{1} \times L^{2}}
$$


Therefore we get

$$
\left\|\partial_{x^{\prime}}^{k} \partial_{x_{n}}^{l} \mathscr{U}_{3}^{(0)}(t, s) u_{0}\right\|_{2}^{2} \leq C(t-s)^{-\frac{n+1}{2}-k}\left(\left\|\phi_{0}\right\|_{L^{1}}^{2}+\left\|u_{0}\right\|_{L^{1}\left(\mathbb{R}^{n-1} ; H^{1}(0,1) \times L^{2}(0,1)\right)}^{2}\right) .
$$

As for $\left\|\partial_{x^{\prime}}^{k} \partial_{x_{n}}^{l} \mathscr{U}_{1}^{(0)}(t, s) u_{0}\right\|_{2}^{2}$, since $\operatorname{supp}\left(\chi^{(0)}-1\right) \subset\left\{\left|\xi^{\prime}\right| \geq r_{0}\right\}$, it is easy to see that

$$
\left\|\partial_{x^{\prime}}^{k} \partial_{x_{n}}^{l} \mathscr{U}_{1}^{(0)}(t, s) u_{0}\right\|_{2}^{2} \leq C e^{-\kappa_{1} r_{0}^{2}(t-s)}(t-s)^{-\frac{n-1}{2}-k}\left\|\phi_{0}\right\|_{L^{1}\left(\mathbb{R}^{n}\right)}^{2}
$$

As for $\left\|\partial_{x^{\prime}}^{k} \partial_{x_{n}}^{l} \mathscr{U}_{2}^{(0)}(t, s) u_{0}\right\|_{2}^{2}$, using the mean value theorem $\left|1-e^{f}\right|=\int_{0}^{1}\left|e^{\theta f}\right| d \theta|f|$, we have

$$
\begin{gathered}
\left\|\partial_{x^{\prime}}^{k} \partial_{x_{n}}^{l} \mathscr{U}_{2}^{(0)}(t, s) u_{0}\right\|_{2}^{2} \leq\left\|\partial_{x^{\prime}}^{k} \partial_{x_{n}}^{l} \chi^{(0)} e^{-\left(i \kappa_{0} \xi_{1}+\kappa_{1}\left|\xi^{\prime}\right|^{2}\right)(t-s)}\left(e^{\left(\lambda_{0}\left(\xi^{\prime}\right)+i \kappa_{0} \xi_{1}+\kappa_{1}\left|\xi^{\prime}\right|^{2}\right)(t-s)}-1\right) \widehat{\Pi}^{(0)}(t) \widehat{u}_{0}\right\|_{2}^{2} \\
\leq C \int_{\left|\xi^{\prime}\right| \leq r_{0}} e^{-\kappa_{1}\left|\xi^{\prime}\right|^{2}(t-s)}(t-s)^{2}\left|\xi^{\prime}\right|^{6+2 k} d \xi^{\prime}\left\|\phi_{0}\right\|_{L^{1}\left(R^{n}\right)}^{2} \leq C(t-s)^{-\frac{n+1}{2}-k}\left\|\phi_{0}\right\|_{L^{1}\left(\mathbb{R}^{n}\right)}^{2} .
\end{gathered}
$$

As for $\left\|\partial_{x^{\prime}}^{k} \partial_{x_{n}}^{l} \sigma_{t, s} u^{(0)}(t)\right\|_{2}^{2}$, it is straightforward to see that

$$
\left\|\partial_{x^{\prime}}^{k} \partial_{x_{n}}^{l} \sigma_{t, s} u^{(0)}(t)\right\|_{2}^{2} \leq C(t-s)^{-\frac{n-1}{2}-k}\left\|\phi_{0}\right\|_{L^{1}\left(\mathbb{R}^{n}\right)}^{2} .
$$

So we have that

$$
\mathscr{U}^{(0)}(t, s) u_{0}=\sigma_{t, s} u^{(0)}(t)+\mathscr{U}_{1}^{(0)}(t, s) u_{0}+\mathscr{U}_{2}^{(0)}(t, s) u_{0}+\mathscr{U}_{3}^{(0)}(t, s) u_{0} .
$$

As for $\left\|\mathscr{R}^{(0)}(t, s) u_{0}\right\|_{H^{1}}^{2}$, it is estimated as follows. Let

$$
J\left(\xi^{\prime} ; t, s\right)=\widehat{U}_{\xi^{\prime}}\left(t, t-\tau_{1}\right)\left(I-\widehat{\Pi}\left(\xi^{\prime}\right)\right) \widehat{U}_{\xi^{\prime}}(T, 0)^{m} \widehat{U}_{\xi^{\prime}}\left(s+\tau_{2}, s\right) \widehat{u}_{0} .
$$

Using (5.24), we have

$$
\left|J\left(\xi^{\prime} ; t, s\right)\right|_{H^{1}} \leq C\left|\left(\left(I-\widehat{\Pi}\left(\xi^{\prime}\right)\right) \widehat{U}_{\xi^{\prime}}(T, 0)\right)^{m} \widehat{U}_{\xi^{\prime}}\left(s+\tau_{2}, s\right) \widehat{u}_{0}\right|_{H^{1} \times L^{2}} .
$$

Applying (5.29) with $r_{0}$ and $m_{0}$ from Theorem 5.16 we get

$$
\begin{gathered}
\left|J\left(\xi^{\prime} ; t, s\right)\right|_{H^{1}} \leq C\left|\left(\left(I-\widehat{\Pi}\left(\xi^{\prime}\right)\right) \widehat{U}_{\xi^{\prime}}(T, 0)\right)^{m_{0} \frac{m}{m_{0}}}\right|_{L(X)}\left|\widehat{U}_{\xi^{\prime}}\left(s+\tau_{2}, s\right) \widehat{u}_{0}\right|_{H^{1} \times L^{2}} \\
\leq C\left|\left(\left(I-\widehat{\Pi}\left(\xi^{\prime}\right)\right) \widehat{U}_{\xi^{\prime}}(T, 0)\right)^{m_{0}}\right|_{L(X)}^{\frac{m}{m_{0}}}\left|\widehat{U}_{\xi^{\prime}}\left(s+\tau_{2}, s\right) \widehat{u}_{0}\right|_{H^{1} \times L^{2}} \\
\leq C e^{-\frac{1}{m_{0}} m T}\left|\widehat{U}_{\xi^{\prime}}\left(s+\tau_{2}, s\right) \widehat{u}_{0}\right|_{H^{1} \times L^{2}} .
\end{gathered}
$$

We employ (5.24) again to conclude

$$
\left|J\left(\xi^{\prime} ; t, s\right)\right|_{H^{1}} \leq C e^{-\frac{1}{m_{0}} m T}\left|\widehat{u}_{0}\right|_{H^{1} \times L^{2}} \leq C e^{-\frac{1}{m_{0}}(t-s)}\left|\widehat{u}_{0}\right|_{H^{1} \times L^{2}} .
$$

Finally integrating in $\xi^{\prime}$ we get

$$
\left\|\mathscr{R}^{(0)}(t, s) u_{0}\right\|_{H^{1}}^{2} \leq C e^{-2 d(t-s)} \int_{R^{n-1}}\left|\widehat{u}_{0}\right|_{H^{1} \times L^{2}}^{2} d \xi^{\prime}=C e^{-2 d(t-s)}\left\|u_{0}\right\|_{H^{1} \times L^{2}}^{2},
$$

where $d>0$, which concludes the proof. 


\section{References}

[1] T. Gallay, A. Scheel, Diffusive stability of oscillations in reaction-diffusion systems, ArXiv e-prints, arXiv 0806.4915, 2008.

[2] Y. Kagei, Asymptotic behavior of the semigroup associated with the linearized compressible Navier-Stokes equation in an infinite layer, Publ. Res. Inst. Math. Sci., 43 (2007), pp. 763-794.

[3] Y. Kagei, Asymptotic behavior of solutions of the compressible Navier-Stokes equation around the plane Couette flow, J. Math. Fluid Mech., vol.13 (2011), pp. 1-31.

[4] Y. Kagei, Global existence of solutions to the compressible Navier-Stokes equation around parallel flows, preprint.

[5] Y. Kagei, Asymptotic behavior of solutions of the compressible Navier-Stokes equation around parallel flows, in preparation.

[6] Y. Kagei, Yu Nagafuchi and T. Sudou, Decay estimates on solutions of the linearized compressible Navier-Stokes equation around a Poiseuille type flow, J. Math-for-Ind., 2A (2010), pp.39-56. Correction to "Decay estimates on solutions of the linearized compressible Navier-Stokes equation around a Poiseuille type flow" in J. Math-for-Ind., 2A (2010), pp.39-56 J. Math-for-Ind., 2B (2010), pp.235.

[7] T. Kato, Perturbation Theory for Linear Operators, Springer-Verlag, Berlin, Heidelberg, New York (1980).

[8] A. Matsumura and T. Nishida, Initial boundary value problems for the equations of motion of compressible viscous and heat-conductive fluids, Commun. Math. Phys., 89 (1983), pp. 445-464.

[9] H. Tanabe, Equations of evolution, Translated from Japanese by N. Mugibayashi and H. Haneda, Pitman, London, San Francisco (1979). 


\section{List of MI Preprint Series, Kyushu University}

The Global COE Program

Math-for-Industry Education \& Research Hub

MI

MI2008-1 Takahiro ITO, Shuichi INOKUCHI \& Yoshihiro MIZOGUCHI

Abstract collision systems simulated by cellular automata

MI2008-2 Eiji ONODERA

The intial value problem for a third-order dispersive flow into compact almost

Hermitian manifolds

MI2008-3 Hiroaki KIDO

On isosceles sets in the 4-dimensional Euclidean space

MI2008-4 Hirofumi NOTSU

Numerical computations of cavity flow problems by a pressure stabilized characteristiccurve finite element scheme

MI2008-5 Yoshiyasu OZEKI

Torsion points of abelian varieties with values in nfinite extensions over a padic field

MI2008-6 Yoshiyuki TOMIYAMA

Lifting Galois representations over arbitrary number fields

MI2008-7 Takehiro HIROTSU \& Setsuo TANIGUCHI

The random walk model revisited

MI2008-8 Silvia GANDY, Masaaki KANNO, Hirokazu ANAI \& Kazuhiro YOKOYAMA Optimizing a particular real root of a polynomial by a special cylindrical algebraic decomposition

MI2008-9 Kazufumi KIMOTO, Sho MATSUMOTO \& Masato WAKAYAMA

Alpha-determinant cyclic modules and Jacobi polynomials 
MI2008-10 Sangyeol LEE \& Hiroki MASUDA

Jarque-Bera Normality Test for the Driving Lévy Process of a Discretely Observed Univariate SDE

MI2008-11 Hiroyuki CHIHARA \& Eiji ONODERA

A third order dispersive flow for closed curves into almost Hermitian manifolds

MI2008-12 Takehiko KINOSHITA, Kouji HASHIMOTO and Mitsuhiro T. NAKAO

On the $L^{2}$ a priori error estimates to the finite element solution of elliptic problems with singular adjoint operator

MI2008-13 Jacques FARAUT and Masato WAKAYAMA

Hermitian symmetric spaces of tube type and multivariate Meixner-Pollaczek polynomials

MI2008-14 Takashi NAKAMURA

Riemann zeta-values, Euler polynomials and the best constant of Sobolev inequality

MI2008-15 Takashi NAKAMURA

Some topics related to Hurwitz-Lerch zeta functions

MI2009-1 Yasuhide FUKUMOTO

Global time evolution of viscous vortex rings

MI2009-2 Hidetoshi MATSUI \& Sadanori KONISHI

Regularized functional regression modeling for functional response and predictors

MI2009-3 Hidetoshi MATSUI \& Sadanori KONISHI

Variable selection for functional regression model via the $L_{1}$ regularization

MI2009-4 Shuichi KAWANO \& Sadanori KONISHI

Nonlinear logistic discrimination via regularized Gaussian basis expansions

MI2009-5 Toshiro HIRANOUCHI \& Yuichiro TAGUCHII

Flat modules and Groebner bases over truncated discrete valuation rings 
MI2009-6 Kenji KAJIWARA \& Yasuhiro OHTA

Bilinearization and Casorati determinant solutions to non-autonomous $1+1$ dimensional discrete soliton equations

\section{MI2009-7 Yoshiyuki KAGEI}

Asymptotic behavior of solutions of the compressible Navier-Stokes equation around the plane Couette flow

MI2009-8 Shohei TATEISHI, Hidetoshi MATSUI \& Sadanori KONISHI

Nonlinear regression modeling via the lasso-type regularization

MI2009-9 Takeshi TAKAISHI \& Masato KIMURA

Phase field model for mode III crack growth in two dimensional elasticity

MI2009-10 Shingo SAITO

Generalisation of Mack's formula for claims reserving with arbitrary exponents for the variance assumption

MI2009-11 Kenji KAJIWARA, Masanobu KANEKO, Atsushi NOBE \& Teruhisa TSUDA Ultradiscretization of a solvable two-dimensional chaotic map associated with the Hesse cubic curve

\section{MI2009-12 Tetsu MASUDA}

Hypergeometric T -functions of the q-Painlevé system of type $E_{8}^{(1)}$

MI2009-13 Hidenao IWANE, Hitoshi YANAMI, Hirokazu ANAI \& Kazuhiro YOKOYAMA A Practical Implementation of a Symbolic-Numeric Cylindrical Algebraic Decomposition for Quantifier Elimination

MI2009-14 Yasunori MAEKAWA

On Gaussian decay estimates of solutions to some linear elliptic equations and its applications

MI2009-15 Yuya ISHIHARA \& Yoshiyuki KAGEI

Large time behavior of the semigroup on $L^{p}$ spaces associated with the linearized compressible Navier-Stokes equation in a cylindrical domain 
MI2009-16 Chikashi ARITA, Atsuo KUNIBA, Kazumitsu SAKAI \& Tsuyoshi SAWABE Spectrum in multi-species asymmetric simple exclusion process on a ring

MI2009-17 Masato WAKAYAMA \& Keitaro YAMAMOTO

Non-linear algebraic differential equations satisfied by certain family of elliptic functions

MI2009-18 Me Me NAING \& Yasuhide FUKUMOTO

Local Instability of an Elliptical Flow Subjected to a Coriolis Force

MI2009-19 Mitsunori KAYANO \& Sadanori KONISHI

Sparse functional principal component analysis via regularized basis expansions and its application

MI2009-20 Shuichi KAWANO \& Sadanori KONISHI

Semi-supervised logistic discrimination via regularized Gaussian basis expansions

MI2009-21 Hiroshi YOSHIDA, Yoshihiro MIWA \& Masanobu KANEKO

Elliptic curves and Fibonacci numbers arising from Lindenmayer system with symbolic computations

MI2009-22 Eiji ONODERA

A remark on the global existence of a third order dispersive flow into locally Hermitian symmetric spaces

MI2009-23 Stjepan LUGOMER \& Yasuhide FUKUMOTO

Generation of ribbons, helicoids and complex scherk surface in laser-matter Interactions

MI2009-24 Yu KAWAKAMI

Recent progress in value distribution of the hyperbolic Gauss map

MI2009-25 Takehiko KINOSHITA \& Mitsuhiro T. NAKAO

On very accurate enclosure of the optimal constant in the a priori error estimates for $H_{0}^{2}$-projection 
MI2009-26 Manabu YOSHIDA

Ramification of local fields and Fontaine's property (Pm)

MI2009-27 Yu KAWAKAMI

Value distribution of the hyperbolic Gauss maps for flat fronts in hyperbolic three-space

MI2009-28 Masahisa TABATA

Numerical simulation of fluid movement in an hourglass by an energy-stable finite element scheme

MI2009-29 Yoshiyuki KAGEI \& Yasunori MAEKAWA Asymptotic behaviors of solutions to evolution equations in the presence of translation and scaling invariance

MI2009-30 Yoshiyuki KAGEI \& Yasunori MAEKAWA

On asymptotic behaviors of solutions to parabolic systems modelling chemotaxis

MI2009-31 Masato WAKAYAMA \& Yoshinori YAMASAKI

Hecke's zeros and higher depth determinants

MI2009-32 Olivier PIRONNEAU \& Masahisa TABATA

Stability and convergence of a Galerkin-characteristics finite element scheme of lumped mass type

MI2009-33 Chikashi ARITA

Queueing process with excluded-volume effect

MI2009-34 Kenji KAJIWARA, Nobutaka NAKAZONO \& Teruhisa TSUDA

Projective reduction of the discrete Painlevé system of type $\left(A_{2}+A_{1}\right)^{(1)}$

MI2009-35 Yosuke MIZUYAMA, Takamasa SHINDE, Masahisa TABATA \& Daisuke TAGAMI Finite element computation for scattering problems of micro-hologram using DtN map 
MI2009-36 Reiichiro KAWAI \& Hiroki MASUDA

Exact simulation of finite variation tempered stable Ornstein-Uhlenbeck processes

MI2009-37 Hiroki MASUDA

On statistical aspects in calibrating a geometric skewed stable asset price model

MI2010-1 Hiroki MASUDA

Approximate self-weighted LAD estimation of discretely observed ergodic OrnsteinUhlenbeck processes

MI2010-2 Reiichiro KAWAI \& Hiroki MASUDA

Infinite variation tempered stable Ornstein-Uhlenbeck processes with discrete observations

MI2010-3 Kei HIROSE, Shuichi KAWANO, Daisuke MIIKE \& Sadanori KONISHI Hyper-parameter selection in Bayesian structural equation models

MI2010-4 Nobuyuki IKEDA \& Setsuo TANIGUCHI

The Itô-Nisio theorem, quadratic Wiener functionals, and 1-solitons

MI2010-5 Shohei TATEISHI \& Sadanori KONISHI

Nonlinear regression modeling and detecting change point via the relevance vector machine

MI2010-6 Shuichi KAWANO, Toshihiro MISUMI \& Sadanori KONISHI

Semi-supervised logistic discrimination via graph-based regularization

MI2010-7 Teruhisa TSUDA

UC hierarchy and monodromy preserving deformation

MI2010-8 Takahiro ITO

Abstract collision systems on groups

MI2010-9 Hiroshi YOSHIDA, Kinji KIMURA, Naoki YOSHIDA, Junko TANAKA \& Yoshihiro MIWA

An algebraic approach to underdetermined experiments 
MI2010-10 Kei HIROSE \& Sadanori KONISHI

Variable selection via the grouped weighted lasso for factor analysis models

MI2010-11 Katsusuke NABESHIMA \& Hiroshi YOSHIDA

Derivation of specific conditions with Comprehensive Groebner Systems

MI2010-12 Yoshiyuki KAGEI, Yu NAGAFUCHI \& Takeshi SUDOU

Decay estimates on solutions of the linearized compressible Navier-Stokes equation around a Poiseuille type flow

MI2010-13 Reiichiro KAWAI \& Hiroki MASUDA

On simulation of tempered stable random variates

MI2010-14 Yoshiyasu OZEKI

Non-existence of certain Galois representations with a uniform tame inertia weight

MI2010-15 Me Me NAING \& Yasuhide FUKUMOTO

Local Instability of a Rotating Flow Driven by Precession of Arbitrary Frequency

MI2010-16 Yu KAWAKAMI \& Daisuke NAKAJO

The value distribution of the Gauss map of improper affine spheres

MI2010-17 Kazunori YASUTAKE

On the classification of rank 2 almost Fano bundles on projective space

MI2010-18 Toshimitsu TAKAESU

Scaling limits for the system of semi-relativistic particles coupled to a scalar bose field

MI2010-19 Reiichiro KAWAI \& Hiroki MASUDA

Local asymptotic normality for normal inverse Gaussian Lévy processes with high-frequency sampling

MI2010-20 Yasuhide FUKUMOTO, Makoto HIROTA \& Youichi MIE

Lagrangian approach to weakly nonlinear stability of an elliptical flow 
MI2010-21 Hiroki MASUDA

Approximate quadratic estimating function for discretely observed Lévy driven SDEs with application to a noise normality test

MI2010-22 Toshimitsu TAKAESU

A Generalized Scaling Limit and its Application to the Semi-Relativistic Particles System Coupled to a Bose Field with Removing Ultraviolet Cutoffs

MI2010-23 Takahiro ITO, Mitsuhiko FUJIO, Shuichi INOKUCHI \& Yoshihiro MIZOGUCHI Composition, union and division of cellular automata on groups

MI2010-24 Toshimitsu TAKAESU

A Hardy's Uncertainty Principle Lemma in Weak Commutation Relations of Heisenberg-Lie Algebra

MI2010-25 Toshimitsu TAKAESU

On the Essential Self-Adjointness of Anti-Commutative Operators

MI2010-26 Reiichiro KAWAI \& Hiroki MASUDA

On the local asymptotic behavior of the likelihood function for Meixner Lévy processes under high-frequency sampling

MI2010-27 Chikashi ARITA \& Daichi YANAGISAWA

Exclusive Queueing Process with Discrete Time

MI2010-28 Jun-ichi INOGUCHI, Kenji KAJIWARA, Nozomu MATSUURA \& Yasuhiro OHTA

Motion and Bäcklund transformations of discrete plane curves

MI2010-29 Takanori YASUDA, Masaya YASUDA, Takeshi SHIMOYAMA \& Jun KOGURE On the Number of the Pairing-friendly Curves

MI2010-30 Chikashi ARITA \& Kohei MOTEGI

Spin-spin correlation functions of the $q$-VBS state of an integer spin model

MI2010-31 Shohei TATEISHI \& Sadanori KONISHI

Nonlinear regression modeling and spike detection via Gaussian basis expansions 
MI2010-32 Nobutaka NAKAZONO

Hypergeometric $\tau$ functions of the $q$-Painlevé systems of type $\left(A_{2}+A_{1}\right)^{(1)}$

MI2010-33 Yoshiyuki KAGEI

Global existence of solutions to the compressible Navier-Stokes equation around parallel flows

MI2010-34 Nobushige KUROKAWA, Masato WAKAYAMA \& Yoshinori YAMASAKI Milnor-Selberg zeta functions and zeta regularizations

MI2010-35 Kissani PERERA \& Yoshihiro MIZOGUCHI

Laplacian energy of directed graphs and minimizing maximum outdegree algorithms

MI2010-36 Takanori YASUDA

CAP representations of inner forms of $S p(4)$ with respect to Klingen parabolic subgroup

MI2010-37 Chikashi ARITA \& Andreas SCHADSCHNEIDER

Dynamical analysis of the exclusive queueing process

MI2011-1 Yasuhide Fukumoto \& Alexander B. Samokhin

Singular electromagnetic modes in an anisotropic medium

MI2011-2 Hiroki Kondo, Shingo Saito \& Setsuo Taniguchi

Asymptotic tail dependence of the normal copula

MI2011-3 Takehiro Hirotsu, Hiroki Kondo, Shingo Saito, Takuya Sato, Tatsushi Tanaka \& Setsuo Taniguchi

Anderson-Darling test and the Malliavin calculus

MI2011-4 Hiroshi Inoue, Shohei Tateishi \& Sadanori Konishi

Nonlinear regression modeling via Compressed Sensing

MI2011-5 Hiroshi Inoue

Implications in Compressed Sensing and the Restricted Isometry Property

MI2011-6 Daeju Kim \& Sadanori Konishi

Predictive information criterion for nonlinear regression model based on basis expansion methods

MI2011-7 Shohei Tateishi, Chiaki Kinjo \& Sadanori Konishi

Group variable selection via relevance vector machine 
MI2011-8 Jan Brezina \& Yoshiyuki Kagei

Decay properties of solutions to the linearized compressible Navier-Stokes equation around time-periodic parallel flow 